\title{
OPERADIC TWISTING - WITH AN APPLICATION TO DELIGNE'S CONJECTURE
}

\author{
VASILY DOLGUSHEV AND THOMAS WILLWACHER
}

To the memory of Jean-Louis Loday

\begin{abstract}
We study categorial properties of the operadic twisting functor Tw. In particular, we show that $\mathrm{Tw}$ is a comonad. Coalgebras of this comonad are operads for which a natural notion of twisting by Maurer-Cartan elements exists. We give a large class of examples, including the classical cases of the Lie, associative and Gerstenhaber operads, and their infinity-counterparts $\mathrm{Lie}_{\infty}, \mathrm{As}_{\infty}, \mathrm{Ger}_{\infty}$. We also show that $\mathrm{Tw}$ is well behaved with respect to the homotopy theory of operads. As an application we show that every solution of Deligne's conjecture is homotopic to a solution that is compatible with twisting.
\end{abstract}

\section{Contents}

1. Introduction

1.1. Structure of the paper

Memorial note

Acknowledgements

2. Preliminaries

2.1. Preliminaries on operads, pseudo-operads and their dual versions

2.2. Conventions related to trees

2.3. The cobar construction and the convolution Lie algebra

3. Twisting of operads

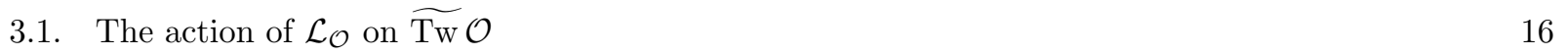

3.2. Algebras over $\mathrm{Tw} \mathcal{O}$

3.3. Example: The dg operad $\mathrm{Tw} \Lambda \mathrm{Lie}_{\infty}$

3.4. A useful modification $\mathrm{Tw}^{\oplus} \mathcal{O}$

3.5. More general version of twisting 26

4. Categorial properties of twisting $\quad 26$

4.1. Tw as a comonad 26

4.2. Coalgebras over the comonad Tw 31

4.3. $\mathrm{Ger}_{\infty}$ is canonically a Tw-coalgebra 32

5. Homotopy theoretic properties of the twisting procedure 38

5.1. The distributive law and the functor $\mathrm{Tw}$

6. Twisting $\mathcal{O}$-algebra structures by Maurer-Cartan elements 49

7. The operad of brace trees BT 51

7.1. The operad structure on BT 52

8. The operad Tw BT 54 
8.1. The differential on Tw BT 57

9. The braces operad $\mathrm{Br}$ à la Kontsevich-Soibelman 58

9.1. A simpler description of the differential on $\mathrm{Br}$ 59

9.2. The inclusion $\mathrm{Br} \hookrightarrow \mathrm{Tw} \mathrm{BT}$ is a quasi-isomorphism 59

9.3. The dg operad $\mathrm{Br}$ is a homotopy fixed point of $\mathrm{Tw}$

9.4. $\mathrm{Br}$ is a $\mathrm{Tw}$-coalgebra 61

10. Every solution of Deligne's conjecture is homotopic to a one that is compatible with twisting 62

10.1. The proof of Proposition 10.3

10.2. Proof of Corollary $1.2 \quad 67$

Appendix A. The operad Ger 68

Appendix B. Action of the operad Tw BT on the Hochschild cochain complex of an $A_{\infty}$-algebra 69

Appendix C. The equivalent definition of the pre-Lie product $\bullet$ on $\operatorname{Conv}(Q, P)$ in terms of coinvariants 772

Appendix D. A Lemma on a filtered complex 77

Appendix E. $\mathrm{Br}$ is a homotopy $\mathrm{Tw}$ fixed point - combinatorial argument 74

References

\section{INTRODUCTION}

The idea of twisting algebraic objects by Maurer-Cartan elements has been a folklore for a very long time and the original nudge for this idea probably belongs to D. Quillen [29].

For example, let $\mathfrak{g}$ be a differential graded Lie algebra and let $m \in \mathfrak{g}$ be a Maurer-Cartan element, i.e., a degree 1 element satisfying the Maurer-Cartan equation

$$
d m+\frac{1}{2}[m, m]=0 .
$$

Then the twisted Lie algebra $\mathfrak{g}^{m}$ is the graded space $\mathfrak{g}$ with differential $d+[m, \cdot]$ and the same bracket $[\cdot, \cdot]$. In a similar manner the notion of Maurer-Cartan element and twisting can be defined for associative and Gerstenhaber algebras, and their homotopy versions, i.e., $\mathrm{Lie}_{\infty}, \mathrm{As}_{\infty}$ and $\mathrm{Ger}_{\infty}$ algebras (see, e. g. [4], 6], [7. Section 2.4], and [34]).

In this paper we investigate in how far the operation of twisting by Maurer-Cartan elements can be defined in general for algebras $A$ over an operad $\mathcal{O}$. We assume that there is a map from the $\mathrm{Lie}_{\infty}$ operad (or a degree shifted version thereof) into $\mathcal{O}$. This endows $A$ with a $\mathrm{Lie}_{\infty}$ algebra structure. A Maurer-Cartan element $m$ of $A$ is then defined as a Maurer-Cartan element of the $\operatorname{Lie}_{\infty}$ algebra $A$. We may twist the Lie $_{\infty}$ algebra $A$ by the Maurer-Cartan element $m$, but in general the twisted Lie $_{\infty}$ algebra structure does not necessarily lift to an $\mathcal{O}$ algebra structure in a natural way.

We provide an algebraic formalism to deal with operads $\mathcal{O}$ for which there is a natural lift. In fact, we show in Theorem 4.7 that the operadic twisting functor $\mathrm{Tw}$ introduced in 33 ] is naturally equipped with the structure of a comonad. This comonad furthermore has the following properties 1 .

\footnotetext{
${ }^{1}$ Here we postpone the precise statements to later sections, since they require terminology introduced in the definition of Tw .
} 
- The twisting procedure by Maurer-Cartan elements can be defined for algebras over an operad which is, in turn, a coalgebra for the comonad Tw, see Section 6 .

- A map $\mathcal{O} \rightarrow \mathcal{O}^{\prime}$ of operads induces a functor from the category of $\mathcal{O}^{\prime}$-algebras to the category of $\mathcal{O}$-algebras. If $\mathcal{O} \rightarrow \mathcal{O}^{\prime}$ gives rise to a map of Tw-coalgebras, then this functor is compatible with the operation of twisting by Maurer-Cartan elements, see Theorem 6.1.

- The functor Tw preserves quasi-isomorphisms between operads, see Theorem 5.1 .

Concretely, the twisted operad $\operatorname{Tw} \mathcal{O}$ is a completion of the operad generated by $\mathcal{O}$ and a unary element, representing the Maurer-Cartan element, with a suitable differential. A pair consisting of an $\mathcal{O}$ algebra $A$ and a Maurer-Cartan element $m$ defines a Tw $\mathcal{O}$ algebra, and moreover the category $\operatorname{Alg}_{\mathcal{O}}^{\mathrm{MC}}$ of such pairs is isomorphic to the category of (filtered) Tw $\mathcal{O}$ algebras, see Theorem 3.10. A Tw coalgebra structure on $\mathcal{O}$ is described by map $\mathcal{O} \rightarrow \operatorname{Tw} \mathcal{O}$. The twisted $\mathcal{O}$ algebra $A^{m}$ is obtained by pulling back the Tw $\mathcal{O}$ algebra structure along this map.

The axioms for a coalgebra over a comonad translate into the statements that twisting with the zero Maurer-Cartan element retains the original algebra and twisting by a Maurer-Cartan element $m$, followed by twisting by a Maurer-Cartan element $m^{\prime}$ is the same as twisting by the Maurer-Cartan element $m+m^{\prime}$.

We apply the developed machinery to study solutions of (our version of) the Deligne conjecture. By this we mean, abusing notation, a quasi-isomorphism

$$
F: \mathrm{Ger}_{\infty} \rightarrow \mathrm{Br}
$$

from the operad $\mathrm{Ger}_{\infty}$ governing homotopy Gerstenhaber algebras to the braces operad $\mathrm{Br}$.

Both operads $\mathrm{Ger}_{\infty}$ and $\mathrm{Br}$ are $\mathrm{Tw}$-coalgebras, i.e., their algebras can be twisted by Maurer-Cartan elements. However, in general, a quasi-isomorphism (1.1) does not need to be a morphism of Tw-coalgebras.

Using our machinery, we establish that every homotopy class of a quasi-isomorphism (1.1) has at least one representative which respects the structures of $\mathrm{Tw}$-coalgebras on $\mathrm{Ger}_{\infty}$ and $\mathrm{Br}$. More precisely,

Theorem 1.1. Suppose that $F: \mathrm{Ger}_{\infty} \rightarrow \mathrm{Br}$ is a quasi-isomorphism of $d g$ operads. Then there exists a quasi-isomorphism of $d g$ operads $F^{\prime}: \mathrm{Ger}_{\infty} \rightarrow \mathrm{Br}$ such that

- $F^{\prime}$ is a homomorphism of $\mathrm{Tw}$-coalgebras, and

- $F^{\prime}$ is homotopy equivalent to $F$.

Furthermore, the morphism $F^{\prime}$ is given by the explicit formula 10.1.

Let us recall that, since the operad $\mathrm{Br}$ acts on the Hochschild cochain complex $C^{\bullet}(A)$ of an $A_{\infty}$-algebra $A$, a quasi-isomorphism (1.1) gives a $\operatorname{Ger}_{\infty}$-structure on $C^{\bullet}(A)$. Theorem 1.1 has an interesting consequence related to this $\mathrm{Ger}_{\infty}$-structure which we will describe now.

Let $A$ be an $A_{\infty}$-algebra and let $F_{A}$ denote the composition

$$
F_{A}=\mathfrak{a}_{A} \circ F: \operatorname{Ger}_{\infty} \rightarrow \operatorname{End}_{C} \cdot(A),
$$

where $\operatorname{End}_{C} \cdot(A)$ is the endomorphism operad of $C^{\bullet}(A)$ and

$$
\mathfrak{a}_{A}: \mathrm{Br} \rightarrow \operatorname{End}_{C} \cdot(A)
$$


is the operad morphism coming from the action of $\mathrm{Br}$ on $C^{\bullet}(A)$. Let us assume, in addition, that the $\mathrm{dg}$ Lie algebra $C^{\bullet}(A)$ carries a complete descending filtration ${ }^{2} \mathcal{F}_{\bullet} C^{\bullet}(A)$ and let $\alpha$ be a Maurer-Cartan element in $\mathcal{F}_{1} C^{\bullet}(A)$.

Such a Maurer-Cartan element gives us a new $A_{\infty}$-structure on $A$ and we denote this new $A_{\infty}$-algebra by $A^{\alpha}$. In addition, the Maurer-Cartan element $\alpha$ can be used to twist the dg Lie algebra structure on $C^{\bullet}(A)$ and form a new cochain complex

$$
C^{\bullet}(A)^{\alpha}
$$

It is easy to see that the cochain complex $C^{\bullet}(A)^{\alpha}$ coincides with the Hochschild cochain complex $C^{\bullet}\left(A^{\alpha}\right)$ of the new $A_{\infty}$-algebra $A^{\alpha}$. Furthermore, there are two natural ways of obtaining a Ger $_{\infty}$-structure on $C^{\bullet}\left(A^{\alpha}\right)=C^{\bullet}(A)^{\alpha}$. First, we may compose the operad morphism

$$
\mathfrak{a}_{A^{\alpha}}: \mathrm{Br} \rightarrow \operatorname{End}_{C} \bullet\left(A^{\alpha}\right)
$$

with $F: \mathrm{Ger}_{\infty} \rightarrow \mathrm{Br}$ and, second, we may twist $F_{A}(1.2)$ by $\alpha$ and obtain

$$
F_{A}^{\alpha}: \operatorname{Ger}_{\infty} \rightarrow \operatorname{End}_{C} \cdot(A)^{\alpha} .
$$

We claim that

Corollary 1.2. For every solution $F$ (1.1) of Deligne's conjecture, the maps $F_{A^{\alpha}}$ and $F_{A}^{\alpha}$ are homotopic. Furthermore, if the morphism $F$ is compatible with the coalgebra structures on $\mathrm{Ger}_{\infty}$ and $\mathrm{Br}$ over $\mathrm{Tw}$, then $F_{A^{\alpha}}$ and $F_{A}^{\alpha}$ coincide.

For the proof of this corollary we refer the reader to Section 10.2 .

1.1. Structure of the paper. In Section 2, we fix notation and recall some facts about operads and their dual versions. In Section 3, we introduce the functor Tw 33] and give an alternative description of the category of algebras over an operad $\mathrm{Tw} \mathcal{O}$. In this section, we also describe in detail the dg operad Tw $\Lambda \operatorname{Lie}_{\infty}$.

Section 4 is devoted to categorical properties of the functor $\mathrm{Tw}$. We show that the functor Tw forms a comonad on the under-category $\Lambda \mathrm{Lie}_{\infty} \downarrow$ Operads. At the end of this section, we prove that the dg operad $\mathrm{Ger}_{\infty}$ governing homotopy Gerstenhaber algebras has the canonical coalgebra structure over the comonad Tw .

In Section 5, we describe homotopy theoretic properties of the functor Tw and introduce the notion of homotopy fixed point for Tw (see Definition 5.3). In this section, we also describe a large class of dg operads which are simultaneously Tw-coalgebras and homotopy fixed points for Tw . This class includes the classical operads Lie, Ger and the operad governing Poisson algebras. At the end of this section, we show that the operads $\mathrm{As}$ and $\mathrm{As}_{\infty}$ are $\mathrm{Tw}$-coalgebras and are homotopy fixed points for $\mathrm{Tw}$.

In Section 6 we consider a dg operad $\mathcal{O}$ which carries a coalgebra structure over $\mathrm{Tw}$. We introduce the notion of twisting by Maurer-Cartan elements for $\mathcal{O}$-algebras and describe categorial and homotopy theoretical properties of this twisting procedure.

Sections 7, 8 and 9 treat the braces operad $\mathrm{Br}$. In this section, we show how to recover the $\mathrm{dg}$ operad $\mathrm{Br}$ (essentially) as the twisted version of a simpler operad BT. The proofs of Theorem 1.1 and Corollary 1.2 are given in Section 10

\footnotetext{
${ }^{2}$ Such a filtration may come from a formal deformation parameter $\varepsilon$ which enters the game when we extend the base field to the ring $\mathbb{K}[[\varepsilon]]$.
} 
In Appendix $\mathrm{A}$ we recall the operad Ger governing Gerstenhaber algebras and, in Appendix $\mathrm{B}$, we describe in detail the action of the operads BT, Tw BT and $\mathrm{Br}$ on the Hochschild cochain complex $C^{\bullet}(A)$ of an $A_{\infty^{-}}$ algebra $A$. Appendices $\mathrm{C}$ and $\mathrm{D}$ are devoted to two auxiliary technical statements. In Appendix $\mathrm{E}$, we sketch a different proof of the fact that the braces operad $\mathrm{Br}$ is a homotopy fixed point for $\mathrm{Tw}$.

Memorial note. With great sadness, we learned that Jean-Louis Loday died as a consequence of an accident on June 6, 2012. He is widely known for his contributions to algebraic $K$-theory, for his research linking cyclic homology, $K$-theory, and combinatorics. His foundational work on operads has and will have a lasting impact on the development of algebra and topology. We devote our paper to the memory of this great mathematician.

Acknowledgements. We would like to thank Martin Markl, Ezra Getzler, Dmitry Tamarkin and Bruno Vallette for helpful discussions. V.D. acknowledges the NSF grants DMS-0856196, DMS-1161867 and the grant FASI RF 14.740.11.0347. V.D.'s NSF grant DMS-0856196 was supported by American Recovery and Reinvestment Act (ARRA) and it could not be transferred to Temple University from the UC Riverside by ARRA regulations. V.D. would like to thank Charles Greer, Tim LeFort, and Charles Louis from the UCR Office of Research for allowing V.D. to save this grant by creating a subaward from the UC Riverside to Temple University. V.D. also thanks John Baez for serving formally as the PI on DMS-0856196 starting July 1, 2010. Part of this work was conducted while T.W. held a junior fellowship of the Harvard Society of Fellows. T.W. furthermore acknowledges partial support of the Swiss National Science Foundation, grants PDAMP2_137151 and 200021_150012.

Both authors are very grateful to the referee for her/his suggestions and patience in reading this long paper, and for providing the references [10, 19, 27] that the authors were unaware of.

\section{Preliminaries}

The underlying field $\mathbb{K}$ has characteristic zero. For a set $X$ we denote by $\mathbb{K}\langle X\rangle$ the $\mathbb{K}$-vector space of finite linear combinations of elements in $X$.

The notation $S_{n}$ is reserved for the symmetric group on $n$ letters and $\mathrm{Sh}_{p_{1}, \ldots, p_{k}}$ denotes the subset of $\left(p_{1}, \ldots, p_{k}\right)$-shuffles in $S_{n}$, i.e. $\mathrm{Sh}_{p_{1}, \ldots, p_{k}}$ consists of elements $\sigma \in S_{n}, n=p_{1}+p_{2}+\cdots+p_{k}$ such that

$$
\begin{gathered}
\sigma(1)<\sigma(2)<\cdots<\sigma\left(p_{1}\right), \\
\sigma\left(p_{1}+1\right)<\sigma\left(p_{1}+2\right)<\cdots<\sigma\left(p_{1}+p_{2}\right), \\
\cdots \\
\sigma\left(n-p_{k}+1\right)<\sigma\left(n-p_{k}+2\right)<\cdots<\sigma(n) .
\end{gathered}
$$

The underlying symmetric monoidal category $\mathfrak{C}$ is either the category grVect $\mathbb{K}_{\mathbb{K}}$ of $\mathbb{Z}$-graded $\mathbb{K}$-vector spaces or the category $\mathrm{Ch}_{\mathbb{K}}$ of (possibly) unbounded cochain complexes of $\mathbb{K}$-vector spaces. We frequently use the ubiquitous combination "dg" (differential graded) to refer to algebraic objects in $\mathrm{Ch}_{\mathbb{K}}$. For a homogeneous vector $v$ in a cochain complex, $|v|$ denotes the degree of $v$. Furthermore, we denote by $\mathbf{s}\left(\right.$ resp. $\mathbf{s}^{-1}$ ) the operation of suspension (resp. desuspension) on grVect $_{\mathbb{K}}$ or $\mathrm{Ch}_{\mathbb{K}}$.

For a groupoid $\mathcal{G}$ the notation $\pi_{0}(\mathcal{G})$ is reserved for the set of its isomorphism classes. 
2.1. Preliminaries on operads, pseudo-operads and their dual versions. By a collection we mean the sequence $\{P(n)\}_{n \geq 0}$ of objects of the underlying symmetric monoidal category $\mathfrak{C}$ such that for each $n$, the object $P(n)$ is equipped with a left action of the symmetric group $S_{n}$.

The notation Lie (resp. As, Com, Ger) is reserved for the operad governing Lie algebras (resp. associative algebras without unit, commutative (and associative) algebras without unit, Gerstenhaber algebras without unit). Dually, the notation coLie (resp. coAs, coCom) is reserved for the cooperad governing Lie coalgebras (resp. coassociative coalgebras without counit, cocommutative (and coassociative) coalgebras without counit).

For an operad $\mathcal{O}($ resp. a cooperad $\mathcal{C})$ and a cochain complex $V$, the notation $\mathcal{O}(V)$ (resp. $\mathcal{C}(V))$ is reserved for the free $\mathcal{O}$-algebra (resp. cofree $\mathcal{C}$-coalgebra). Namely,

$$
\begin{aligned}
& \mathcal{O}(V):=\bigoplus_{n \geq 0}\left(\mathcal{O}(n) \otimes V^{\otimes n}\right)_{S_{n}}, \\
& \mathcal{C}(V):=\bigoplus_{n \geq 0}\left(\mathcal{C}(n) \otimes V^{\otimes n}\right)^{S_{n}} .
\end{aligned}
$$

For an operad $\mathcal{O}$, we denote by $\mathrm{Alg}_{\mathcal{O}}$ the category of $\mathcal{O}$-algebras.

Let $a_{1}, a_{2}, \ldots, a_{n}$ be degree 0 dummy variables and $\mathcal{O}$ be a dg operad. It is clear that $\mathcal{O}(n)$ is naturally identified with the subspace of the free $\mathcal{O}$-algebra

$$
\mathcal{O}\left(\mathbb{K}\left\langle a_{1}, a_{2}, \ldots, a_{n}\right\rangle\right)
$$

spanned by $\mathcal{O}$-monomials in which each variable from the set $\left\{a_{1}, a_{2}, \ldots, a_{n}\right\}$ appears exactly once. We often use this identification in our paper.

Let us denote by $*$ the following collection $\left(\right.$ in $\left.\mathrm{Ch}_{\mathbb{K}}\right)$

$$
*(n)= \begin{cases}\mathbb{K} & \text { if } n=1, \\ \mathbf{0} & \text { otherwise. }\end{cases}
$$

It is easy to see that $*$ is equipped with the unique structure of an operad and a unique structure of a cooperad.

Recall that an operad $\mathcal{O}$ (resp. a cooperad $\mathcal{C}$ ) is called augmented (resp. coaugmented) if it comes with an operad map $\mathcal{O} \rightarrow *($ resp. a cooperad map $* \rightarrow \mathcal{C})$. For an augmented operad $\mathcal{O}$ (resp. a coaugmented cooperad $\mathcal{C}$ ) the notation $\mathcal{O}_{\circ}\left(\right.$ resp. $\mathcal{C}_{\circ}$ ) is reserved for the kernel (resp. the cokernel) of the augmentation (resp. coaugmentation).

Recall that a pseudo-operad ${ }^{3}$ (resp. pseudo-cooperad) is vaguely "an operad (resp. a cooperad) without the unit (resp. counit) axiom". For the more precise definition of a pseudo-operad (resp. a pseudo-cooperad) we refer the reader to [8, Sections 3.2,3.4] or [25, Section 1.3]. We remark that for every augmented operad $\mathcal{O}$ (resp. coaugmented cooperad $\mathcal{C}$ ) the kernel of the augmentation $\mathcal{O}_{\circ}$ (resp. the cokernel of the coaugmentation $\mathcal{C}_{\circ}$ ) is naturally a pseudo-operad (resp. pseudo-cooperad). In fact, due to 23, Proposition 21], the assignment $\mathcal{O} \mapsto \mathcal{O}_{\circ}$ (resp. $\mathcal{C} \mapsto \mathcal{C}_{\circ}$ ) upgrades to an equivalence between the category of augmented operads (resp. coaugmented cooperads) and the category of pseudo-operads (resp. pseudo-cooperads).

\footnotetext{
${ }^{3}$ Note that a pseudo-operad is not a non-unital operad. For more details about this subtlety, see paper 23] in which a pseudo-operad is called a non-unital Markl's operad.
} 
For a pseudo-operad (or an operad) $P$ in $\mathrm{Ch}_{\mathbb{K}}$ we denote by $\circ_{i}$ the $i$-th elementary insertion ${ }^{4}$

$$
\circ_{i}: P(n) \otimes P(k) \rightarrow P(n+k-1)
$$

Here $n \geq 1, k \geq 0$, and $1 \leq i \leq n$.

For an operad (resp. a cooperad) $P$ in $\mathrm{Ch}_{\mathbb{K}}$ we denote by $\Lambda P$ the operad (resp. the cooperad) with the spaces of $n$-ary operations:

$$
\Lambda P(n)=\mathbf{s}^{1-n} P(n) \otimes \operatorname{sgn}_{n},
$$

where $\operatorname{sgn}_{n}$ denotes the sign representation of $S_{n}$. For example, an algebra over $\Lambda$ Lie is a graded vector space $V$ equipped with the binary operation:

$$
\{,\}: V \otimes V \rightarrow V
$$

of degree -1 satisfying the identities:

$$
\begin{gathered}
\left\{v_{1}, v_{2}\right\}=(-1)^{\left|v_{1}\right|\left|v_{2}\right|}\left\{v_{2}, v_{1}\right\} \\
\left\{\left\{v_{1}, v_{2}\right\}, v_{3}\right\}+(-1)^{\left|v_{1}\right|\left(\left|v_{2}\right|+\left|v_{3}\right|\right)}\left\{\left\{v_{2}, v_{3}\right\}, v_{1}\right\}+(-1)^{\left|v_{3}\right|\left(\left|v_{1}\right|+\left|v_{2}\right|\right)}\left\{\left\{v_{3}, v_{1}\right\}, v_{2}\right\}=0,
\end{gathered}
$$

where $v_{1}, v_{2}, v_{3}$ are homogeneous vectors in $V$.

Ger $^{\vee}$ denotes the Koszul dual cooperad [11], [14, [15], 22] for Ger. It is known [18] that

$$
\mathrm{Ger}^{\vee}=\Lambda^{2} \mathrm{Ger}^{*}
$$

where Ger* is obtained from the operad Ger by taking the linear dual. In other words, algebras over the linear dual $\left(\mathrm{Ger}^{\vee}\right)^{*}$ are very much like Gerstenhaber algebras except that the bracket carries degree 1 and the multiplication carries degree 2 .

2.1.1. Intrinsic derivations of an operad. Let $P$ be a $\mathrm{dg}$ operad. Then the operation $\circ_{1}$ equips $P(1)$ with a structure of an associative algebra. We consider $P(1)$ as a Lie algebra with the Lie bracket being the (graded) commutator and we claim that

Proposition 2.1. The formula

$$
\delta_{b}(a)=b \circ_{1} a-(-1)^{|a||b|} \sum_{i=1}^{n} a \circ_{i} b
$$

with

$$
b \in P(1), \quad \text { and } \quad a \in P(n)
$$

defines an operadic derivation of $P$ for every $b \in P(1)$. Furthermore, for every pair $b_{1}, b_{2} \in P(1)$, we have

$$
\left[\delta_{b_{1}}, \delta_{b_{2}}\right]=\delta_{\left[b_{1}, b_{2}\right]}
$$

For the proof of this proposition we refer the reader to [8, Section 6.1]. An operadic derivation of the form 2.7 is called intrinsic.

\footnotetext{
${ }^{4}$ Following the general convention, the numbers $n$ and $k$ are suppressed from the notation $\circ_{i}$.
} 
2.2. Conventions related to trees. By a tree we mean a connected graph without cycles with a marked vertex called the root. In this paper, we assume that the root of every tree has valency 1 (such trees are sometimes called planted). The edge adjacent to the root is called the root edge. Non-root vertices of valency 1 are called leaves. A vertex is called internal if it is neither a root nor a leaf. We always orient trees in the direction towards the root. Thus every internal vertex has at least 1 incoming edge and exactly 1 outgoing edge. An edge adjacent to a leaf is called external. A tree $\mathbf{t}$ is called planar if, for every internal vertex $v$ of $\mathbf{t}$, the set of edges terminating at $v$ carries a total order.

Let us recall (see [8, Section 2]) that for every planar tree $\mathbf{t}$ the set $V(\mathbf{t})$ of all its vertices is equipped with a natural total order such that the root is the smallest vertex of the tree.

We have an obvious bijection between the set of edges $E(\mathbf{t})$ of a tree $\mathbf{t}$ and the subset of vertices:

$$
V(\mathbf{t}) \backslash\{\text { root vertex }\} .
$$

This bijection assigns to a vertex $v$ in $(2.8)$ its outgoing edge. Thus the canonical total order on the set (2.8) gives us a natural total order on the set of edges $E(\mathbf{t})$.

For a non-negative integer $n$, an $n$-labeled planar tree $\mathbf{t}$ is a planar tree equipped with an injective map

$$
\mathfrak{l}:\{1,2, \ldots, n\} \rightarrow L(\mathbf{t})
$$

from the set $\{1,2, \ldots, n\}$ to the set $L(\mathbf{t})$ of leaves of $\mathbf{t}$. Although the set $L(\mathbf{t})$ has a natural total order we do not require that the map $(2.9)$ is monotonous.

The set $L(\mathbf{t})$ of leaves of an $n$-labeled planar tree $\mathbf{t}$ splits into the disjoint union of the image $\mathfrak{l}(\{1,2, \ldots, n\})$ and its complement. We call leaves in the image of $\mathfrak{l}$ labeled.

A vertex $x$ of an $n$-labeled planar tree $\mathbf{t}$ is called nodal if it is neither the root, nor a labeled leaf. We denote by $V_{\text {nod }}(\mathbf{t})$ the set of all nodal vertices of $\mathbf{t}$. Keeping in mind the canonical total order on the set of all vertices of $\mathbf{t}$ we can say things like "the first nodal vertex", "the second nodal vertex", and "the $i$-th nodal vertex".

It is convenient to talk about (co)operads and pseudo-(co)operads using the groupoid $\operatorname{Tree}(n)$ of $n$-labeled planar trees. Objects of Tree $(n)$ are $n$-labeled planar trees and morphisms are non-planar isomorphisms of the corresponding (non-planar) trees compatible with labeling. The groupoid Tree $(n)$ is equipped with an obvious left action of the symmetric group $S_{n}$.

Following [8, Section 3.2, 3.4], for an $n$-labelled planar tree $\mathbf{t}$ and pseudo-operad $P$ (resp. pseudo-cooperad $Q)$ the notation $\mu_{\mathbf{t}}\left(\right.$ resp. the notation $\Delta_{\mathbf{t}}$ ) is reserved for the multiplication map

$$
\mu_{\mathbf{t}}: P\left(r_{1}\right) \otimes P\left(r_{2}\right) \otimes \cdots \otimes P\left(r_{k}\right) \rightarrow P(n)
$$

and the comultiplication map

$$
\Delta_{\mathbf{t}}: Q(n) \rightarrow Q\left(r_{1}\right) \otimes Q\left(r_{2}\right) \otimes \cdots \otimes Q\left(r_{k}\right) .
$$

respectively. Here, $k$ is the number of nodal vertices of the planar tree $\mathbf{t}$ and $r_{i}$ is the number of edges (of t) which terminate at the $i$-th nodal vertex of $\mathbf{t}$.

For example, if $\mathbf{t}_{n, k, i}$ is the labeled planar tree shown on figure ${ }^{5} 2.1$ then the map

$$
\mu_{\mathbf{t}_{n, k, i}}: P(n) \otimes P(k) \rightarrow P(n+k-1)
$$

is precisely the $i$-th elementary insertion 2.4.

\footnotetext{
${ }^{5}$ On figures, small white circles are used for nodal vertices and small black circles are used for all the remaining vertices.
} 


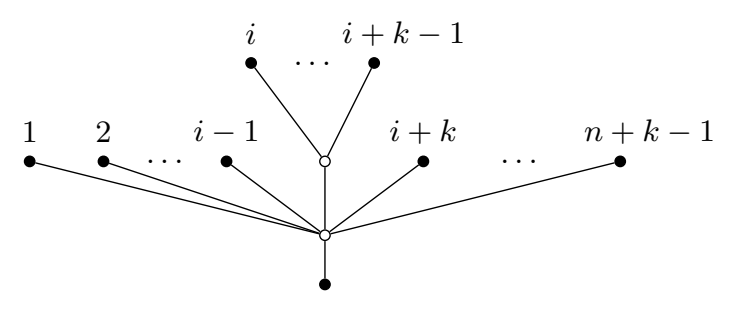

FIG. 2.1. The $(n+k-1)$-labeled planar tree $\mathbf{t}_{n, k, i}$

Let $\sigma$ be an element in $S_{n+k-1}$ and $\sigma\left(\mathbf{t}_{n, k, i}\right)$ be the $(n+k-1)$-labelled planar tree obtained from $\mathbf{t}_{n, k, i}$ (on figure 2.1 via acting by the permutation $\sigma$. Sometimes it is convenient to use a different notation for the multiplication map $\mu_{\sigma\left(\mathbf{t}^{n, k, i}\right)}$ corresponding to the tree $\sigma\left(\mathbf{t}^{n, k, i}\right)$. More precisely, for a vector $v \in P(n)$ and $w \in P(k)$ of a pseudo-operad $P$ we will use this notation

$$
v(\sigma(1), \ldots \sigma(i-1), w(\sigma(i), \ldots, \sigma(i+k-1)), \sigma(i+k), \ldots, \sigma(n+k-1)):=\mu_{\mathbf{t}_{\sigma}^{n, k, i}}(v, w) .
$$

The notation $\operatorname{Tree}_{2}(n)$ is reserved for the full sub-groupoid of Tree $(n)$ whose objects are $n$-labelled planar trees with exactly 2 nodal vertices. It is not hard to see that every object in $\operatorname{Tree}_{2}(n)$ has at most $n+1$ leaves. Furthermore, isomorphism classes of $\operatorname{Tree}_{2}(n)$ are in bijection with the union

$$
\bigsqcup_{0 \leq p \leq n} \operatorname{Sh}_{p, n-p}
$$

where $\mathrm{Sh}_{p, n-p}$ denotes the set of $(p, n-p)$-shuffles in $S_{n}$. The bijection assigns to a $(p, n-p)$-shuffle $\tau$ the isomorphism class of the planar tree depicted on figure 2.2 .

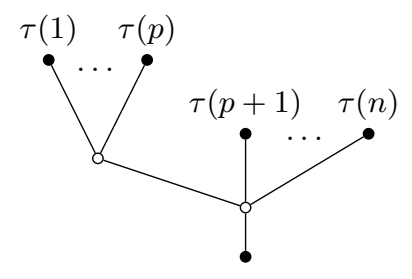

FIG. 2.2. Here $\tau$ is a $(p, n-p)$-shuffle

2.3. The cobar construction and the convolution Lie algebra. In this paper, we denote by $\mathbb{O P}(P)$ the free operad generated by a collection $P$.

Let us recall that the cobar construction Cobar [11, [14, 15], 22] is a functor from the category of coaugmented dg cooperads to the category of augmented dg operads. It is used to construct free resolutions for operads.

More precisely, for a coaugmented dg cooperad $\mathcal{C}$,

$$
\operatorname{Cobar}(\mathcal{C})=\mathbb{O P}\left(\mathbf{s} \mathcal{C}_{\circ}\right)
$$

as the operad in the category $\operatorname{grVect}_{\mathbb{K}}$, and the differential $\partial^{\text {Cobar }}$ on $\operatorname{Cobar}(\mathcal{C})$ is define by the equations

$$
\partial^{\text {Cobar }}=\partial^{\prime}+\partial^{\prime \prime}
$$


with

$$
\partial^{\prime}(X)=-\mathbf{s} \partial_{\mathcal{C}} \mathbf{s}^{-1} X
$$

and

$$
\partial^{\prime \prime}(X)=-\sum_{z \in \pi_{0}\left(\operatorname{Tree}_{2}(n)\right)}(\mathbf{s} \otimes \mathbf{s})\left(\mathbf{t}_{z} ; \Delta_{\mathbf{t}_{z}}\left(\mathbf{s}^{-1} X\right)\right),
$$

where $X \in \mathbf{s} \mathcal{C}_{\circ}(n), \mathbf{t}_{z}$ is any representative ${ }^{6}$ of the isomorphism class $z \in \pi_{0}\left(\operatorname{Tree}_{2}(n)\right)$, and $\partial_{\mathcal{C}}$ is the differential on $\mathcal{C}$.

Let $P\left(\right.$ resp. $Q$ ) be a pseudo-operad (resp. pseudo-cooperad) in the category $\mathrm{Ch}_{\mathbb{K}}$. Following [19] and [28], we equip the cochain complex

$$
\operatorname{Conv}(Q, P)=\prod_{n \geq 0} \operatorname{Hom}_{S_{n}}(Q(n), P(n)) .
$$

with the binary operation $\bullet$ defined by the formula

$$
\begin{gathered}
f \bullet g(X)=\sum_{z \in \pi_{0}\left(\operatorname{Tree}_{2}(n)\right)} \mu_{\mathbf{t}_{z}}\left(f \otimes g \Delta_{\mathbf{t}_{z}}(X)\right), \\
f, g \in \operatorname{Conv}(Q, P), \quad X \in Q(n),
\end{gathered}
$$

where $\mathbf{t}_{z}$ is any representative of the isomorphism class $z \in \pi_{0}\left(\operatorname{Tree}_{2}(n)\right)$. The axioms of a pseudo-operad (resp. pseudo-cooperad) imply that the right hand side of 2.20 does not depend on the choice of representatives $\mathbf{t}_{z}$.

It follows directly from the definition that the operation $\bullet$ is compatible with the differential on $\operatorname{Conv}(Q, P)$ coming from $Q$ and $P$. Furthermore, the operation $\bullet$ satisfies the axiom of the pre-Lie algebra:

$$
(f \bullet g) \bullet h-f \bullet(g \bullet h)=(-1)^{|g||h|}(f \bullet h) \bullet g-(-1)^{|g||h|} f \bullet(h \bullet g) .
$$

Therefore the bracket

$$
[f, g]=\left(f \bullet g-(-1)^{|f||g|} g \bullet f\right)
$$

satisfies the Jacobi identity, and hence $\operatorname{Conv}(Q, P)$ is a $\operatorname{dg}$ Lie algebra.

We refer to $\operatorname{Conv}(Q, P)$ as the convolution Lie algebra of the pair $(Q, P)$.

We observe that for every dg operad $\mathcal{O}$ the morphism (of dg operads)

$$
F: \operatorname{Cobar}(\mathcal{C}) \rightarrow \mathcal{O}
$$

is uniquely determined by its restriction

$$
\left.F\right|_{\mathbf{s} \mathcal{C}_{\circ}}: \mathbf{s} \mathcal{C}_{\circ} \rightarrow \mathcal{O}
$$

Thus, to every morphism 2.21 we assign a degree 1 element

$$
\alpha_{F} \in \operatorname{Conv}\left(\mathcal{C}_{\circ}, \mathcal{O}\right)
$$

such that

$$
\left.F\right|_{\mathbf{s} \mathcal{C}_{\circ}}=\alpha_{F} \circ \mathbf{s}^{-1}
$$

The construction of the $d g$ Lie algebra $\operatorname{Conv}(\mathcal{C}, \mathcal{O})$ is partially justified by the following statement:

\footnotetext{
${ }^{6}$ The axioms of a pseudo-cooperad imply that the right hand side of 2.18 does not depend on the choice of representatives $\mathbf{t}_{z}$.
} 
Theorem 2.2 (Proposition 5.2, [8]). Let $\mathcal{C}$ be a coaugmented $d g$ cooperad with $\mathcal{C}_{\circ}$ being the cokernel of the coaugmentation. Then, for every $d g$ operad $\mathcal{O}$, the above assignment

$$
F \mapsto \alpha_{F}
$$

is a bijection between the set of maps (of dg operads) (2.21) and Maurer-Cartan elements of the dg Lie algebra

$$
\operatorname{Conv}\left(\mathcal{C}_{\circ}, \mathcal{O}\right)
$$

2.3.1. Example: The $d g$ operad $\Lambda \mathrm{Lie}_{\infty}$. Let us recall from [15, that the dg operad $\Lambda \mathrm{Lie}_{\infty}$ governing homotopy $\Lambda$ Lie-algebras is

$$
\Lambda \mathrm{Lie}_{\infty}=\operatorname{Cobar}\left(\Lambda^{2} \mathrm{coCom}\right),
$$

where coCom is the cooperad governing cocommutative coalgebras without counit.

Let us denote by $1_{n}^{\mathfrak{c}}$ the canonical generator

$$
1_{n}^{\mathfrak{c}}:=\mathbf{s}^{2-2 n} 1 \in \mathbf{s}^{2-2 n} \mathbb{K} \cong \Lambda^{2} \operatorname{coCom}(n) .
$$

Using the identification between isomorphism classes of objects in $\operatorname{Tree}_{2}(n)$ and the set (2.14) of shuffles we get

$$
\partial^{\operatorname{Cobar}}\left(\mathbf{s} 1_{n}^{\mathfrak{c}}\right)=-\sum_{p=2}^{n-1} \sum_{\sigma \in \mathrm{Sh}_{p, n-p}} \sigma\left(\left(\mathbf{s} 1_{n-p+1}^{\mathfrak{c}}\right) \circ_{1}\left(\mathbf{s} 1_{p}^{\mathfrak{c}}\right)\right), \quad n \geq 2 .
$$

Let us also recall from [8, Section 5.2] that the canonical quasi-isomorphism of dg operads

$$
U_{\Lambda \text { Lie }}: \Lambda \operatorname{Lie}_{\infty}=\operatorname{Cobar}\left(\Lambda^{2} \text { coCom }\right) \rightarrow \Lambda \text { Lie }
$$

corresponds to the Maurer-Cartan element

$$
\alpha_{\Lambda \text { Lie }}=\left\{a_{1}, a_{2}\right\} \otimes b_{1} b_{2} \in \operatorname{Conv}\left(\Lambda^{2} \operatorname{coCom}_{\circ}, \Lambda \text { Lie }\right) \cong \prod_{n \geq 2}\left(\Lambda \operatorname{Lie}(n) \otimes \Lambda^{-2} \operatorname{Com}(n)\right)^{S_{n}},
$$

where $\left\{a_{1}, a_{2}\right\}$ (resp. $\left.b_{1} b_{2}\right)$ denotes the natural generator of $\Lambda$ Lie(2) (resp. $\left.\Lambda^{-2} \operatorname{Com}(2)\right)$.

In other words,

$$
U_{\Lambda \text { Lie }}\left(\mathbf{s} 1_{n}^{\mathfrak{c}}\right)= \begin{cases}\left\{a_{1}, a_{2}\right\} & \text { if } n=2 \\ 0 & \text { otherwise }\end{cases}
$$

\section{TWISTING OF OPERADS}

Let $\mathcal{O}$ be a dg operad equipped with a map

$$
\widehat{\varphi}: \Lambda \operatorname{Lie}_{\infty} \rightarrow \mathcal{O}
$$

and $V$ be an algebra over $\mathcal{O}$.

Using the map $\widehat{\varphi}$, we equip $V$ with a $\Lambda \mathrm{Lie}_{\infty}$-structure. If we assume, in addition, that $V$ is equipped with a complete descending filtration

$$
V \supset \mathcal{F}_{1} V \supset \mathcal{F}_{2} V \supset \mathcal{F}_{3} V \supset \ldots, \quad V=\lim _{k} V / \mathcal{F}_{k} V
$$


and the $\mathcal{O}$-algebra structure on $V$ is compatible with this filtration then we may define Maurer-Cartan elements of $V$ as degree 2 elements $\alpha \in \mathcal{F}_{1} V$ satisfying the equation

$$
\partial(\alpha)+\sum_{n \geq 2} \frac{1}{n !}\{\alpha, \alpha, \ldots, \alpha\}_{n}=0
$$

where $\partial$ is the differential on $V$ and $\{\cdot, \cdot, \ldots, \cdot\}_{n}$ are the operations of the $\Lambda$ Lie $_{\infty}$-structure on $V$. Given such a Maurer-Cartan element $\alpha$ we can twist the differential on $V$ and insert $\alpha$ into various $\mathcal{O}$-operations on $V$. This way we get a new algebra structure on $V$. It turns out that this new algebra structure is governed by a dg operad Tw $\mathcal{O}$ which is built from the pair $(\mathcal{O}, \widehat{\varphi})$. This section is devoted to the construction of $\operatorname{Tw} \mathcal{O}$.

First, we recall that $\Lambda \mathrm{Lie}_{\infty}=\operatorname{Cobar}\left(\Lambda^{2} \mathrm{coCom}\right)$. Hence, due to Theorem 2.2, the morphism 3.1 is determined by a Maurer-Cartan element

$$
\varphi \in \operatorname{Conv}\left(\Lambda^{2} \operatorname{coCom}_{\circ}, \mathcal{O}\right) .
$$

The $n$-th space of $\Lambda^{2}$ coCom。 $_{\circ}$ is the trivial $S_{n}$-modules placed in degree $2-2 n$ :

$$
\Lambda^{2} \operatorname{coCom}(n)=\mathbf{s}^{2-2 n} \mathbb{K} .
$$

So we have

$$
\operatorname{Conv}\left(\Lambda^{2} \operatorname{coCom}_{\circ}, \mathcal{O}\right)=\prod_{n \geq 2} \operatorname{Hom}_{S_{n}}\left(\mathbf{s}^{2-2 n} \mathbb{K}, \mathcal{O}(n)\right)=\prod_{n \geq 2} \mathbf{s}^{2 n-2}(\mathcal{O}(n))^{S_{n}} .
$$

For our purposes we will need to extend the $\mathrm{dg}$ Lie algebra $\operatorname{Conv}\left(\Lambda^{2} \operatorname{coCom}, \mathcal{O}\right)$ to

$$
\mathcal{L}_{\mathcal{O}}=\operatorname{Conv}\left(\Lambda^{2} \operatorname{coCom}, \mathcal{O}\right)=\prod_{n \geq 1} \operatorname{Hom}_{S_{n}}\left(\mathbf{s}^{2-2 n} \mathbb{K}, \mathcal{O}(n)\right) .
$$

It is clear that

$$
\mathcal{L}_{\mathcal{O}}=\prod_{n \geq 1} \mathbf{s}^{2 n-2}(\mathcal{O}(n))^{S_{n}} .
$$

For $n, r \geq 1$ we realize the group $S_{r}$ as the following subgroup of $S_{r+n}$

$$
S_{r} \cong\left\{\sigma \in S_{r+n} \mid \sigma(i)=i, \quad \forall i>r\right\} .
$$

In other words, the group $S_{r}$ may be viewed as subgroup of $S_{r+n}$ permuting only the first $r$ letters. We set $S_{0}$ to be the trivial group. Using this embedding of $S_{r}$ into $S_{n+r}$ we introduce the following collection $(n \geq 0)$

$$
\widetilde{\mathrm{Tw}} \mathcal{O}(n)=\prod_{r \geq 0} \operatorname{Hom}_{S_{r}}\left(\mathbf{s}^{-2 r} \mathbb{K}, \mathcal{O}(r+n)\right) .
$$

It is clear that

$$
\widetilde{\mathrm{Tw}} \mathcal{O}(n)=\prod_{r \geq 0} \mathbf{s}^{2 r}(\mathcal{O}(r+n))^{S_{r}} .
$$

To define an operad structure on (3.7) we denote by $1_{r}$ the generator

$$
1_{r}:=\mathbf{s}^{-2 r} 1 \in \mathbf{s}^{-2 r} \mathbb{K} .
$$

Then the identity element $\mathbf{u}$ in $\widetilde{\mathrm{Tw}} \mathcal{O}(1)$ is given by

$$
\mathbf{u}\left(1_{r}\right)= \begin{cases}\mathbf{u}_{\mathcal{O}} & \text { if } r=0, \\ 0 & \text { otherwise }\end{cases}
$$


where $\mathbf{u}_{\mathcal{O}} \in \mathcal{O}(1)$ is the identity element for the operad $\mathcal{O}$. Next, for $f \in \widetilde{\mathrm{Tw}} \mathcal{O}(n)$ and $g \in \widetilde{\mathrm{Tw}} \mathcal{O}(m)$, we define the $i$-th elementary insertion $\circ_{i} 1 \leq i \leq n$ by the formula

$$
f \circ_{i} g\left(1_{r}\right)=\sum_{p=0}^{r} \sum_{\sigma \in \mathrm{Sh}_{p, r-p}} \mu_{\mathbf{t}_{\sigma, i}}\left(f\left(1_{p}\right) \otimes g\left(1_{r-p}\right)\right) .
$$

where the tree $\mathbf{t}_{\sigma, i}$ is depicted on figure 3.1

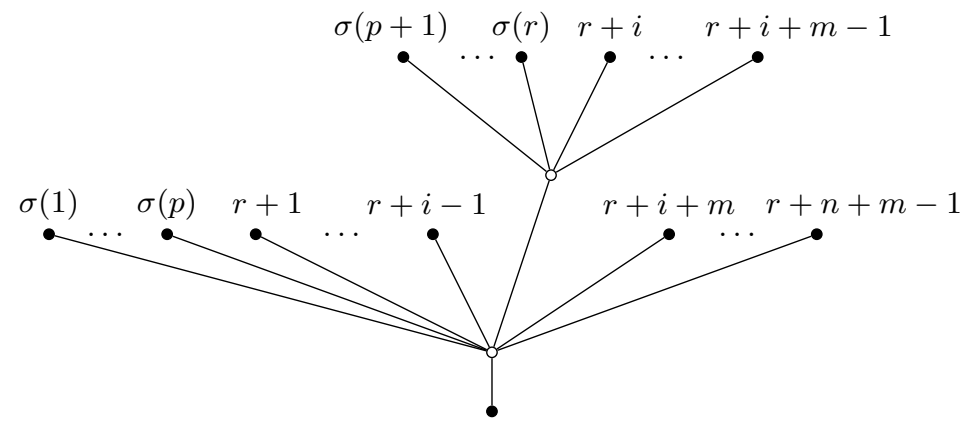

FIG. 3.1. Here $\sigma$ is a $(p, r-p)$-shuffle

Remark 3.1. The operad $\widetilde{\mathrm{Tw}} \mathcal{O}$ is a completed version of the shifted operad $\mathcal{O}[\mathbb{K}]$ introduced in [10, section $1.2 .2]$.

Sometimes it is convenient to use a different but equivalent definition of the $i$-th elementary insertion $\circ_{i}$ for $\widetilde{\mathrm{Tw}} \mathcal{O}$. This definition is given by the formula 7

$$
f \circ_{i} g\left(1_{r}\right)=
$$

$\sum_{p=0}^{r} \sum_{\sigma \in \mathrm{Sh}_{p, r-p}} \sigma\left(f_{p}\left(1, \ldots, p, r+1, \ldots, r+i-1, g_{r-p}(p+1, \ldots, r, r+i, \ldots, r+i+m-1), r+i+m, \ldots, r+n+m-1\right)\right)$, where $f \in \widetilde{\mathrm{Tw}} \mathcal{O}(n), g \in \widetilde{\mathrm{Tw}} \mathcal{O}(m), f_{p}=f\left(1_{p}\right) \in(\mathcal{O}(p+n))^{S_{p}}$ and $g_{q}=g\left(1_{q}\right) \in(\mathcal{O}(q+m))^{S_{q}}$.

To see that the element $f \circ_{i} g\left(1_{r}\right) \in \mathcal{O}(r+n+m-1)$ is $S_{r}$-invariant one simply needs to use the fact that every element $\tau \in S_{r}$ can be uniquely presented as the composition $\tau_{s h} \circ \tau_{p, r-p}$, where $\tau_{s h}$ is a $(p, r-p)$-shuffle and $\tau_{p, r-p} \in S_{p} \times S_{r-p}$.

Let $f \in \widetilde{\mathrm{Tw}} \mathcal{O}(n), g \in \widetilde{\mathrm{Tw}} \mathcal{O}(m), h \in \widetilde{\mathrm{Tw}} \mathcal{O}(k), 1 \leq i \leq n$, and $1 \leq j \leq m$. To check the identity

$$
f \circ_{i}\left(g \circ_{j} h\right)=\left(f \circ_{i} g\right) \circ_{j+i-1} h
$$

we observe that

$$
\begin{aligned}
f \circ_{i}\left(g \circ_{j} h\right)\left(1_{r}\right) & =\sum_{p=0}^{r} \sum_{\sigma \in \operatorname{Sh}_{p, r-p}} \mu_{\mathbf{t}_{\sigma, i}}\left(f\left(1_{p}\right) \otimes\left(g \circ \circ_{j} h\right)\left(1_{r-p}\right)\right) \\
& =\sum_{p_{1}+p_{2}+p_{3}=r} \sum_{\sigma \in \operatorname{Sh}_{p_{1}, p_{2}+p_{3}}} \sum_{\sigma_{1} \in \operatorname{Sh}_{p_{2}, p_{3}}} \mu_{\mathbf{t}_{\sigma, i}} \circ\left(1 \otimes \mu_{\mathbf{t}_{\sigma^{\prime}, j}}\right)\left(f\left(1_{p_{1}}\right) \otimes g\left(1_{p_{2}}\right) \otimes h\left(1_{p_{3}}\right)\right) \\
& =\sum_{p_{2}+p_{3}=r} \sum_{\tau \in \operatorname{Sh}_{p_{1}, p_{2}, p_{3}}} \mu_{\mathbf{t}_{\tau, i, j}}\left(f\left(1_{p_{1}}\right) \otimes g\left(1_{p_{2}}\right) \otimes h\left(1_{p_{3}}\right)\right),
\end{aligned}
$$

where the tree $\mathbf{t}_{\tau, i, j}$ is depicted on figure 3.2 Similar calculations show that

\footnotetext{
${ }^{7}$ Here we use the notation for operadic multiplications 2.13 .
} 


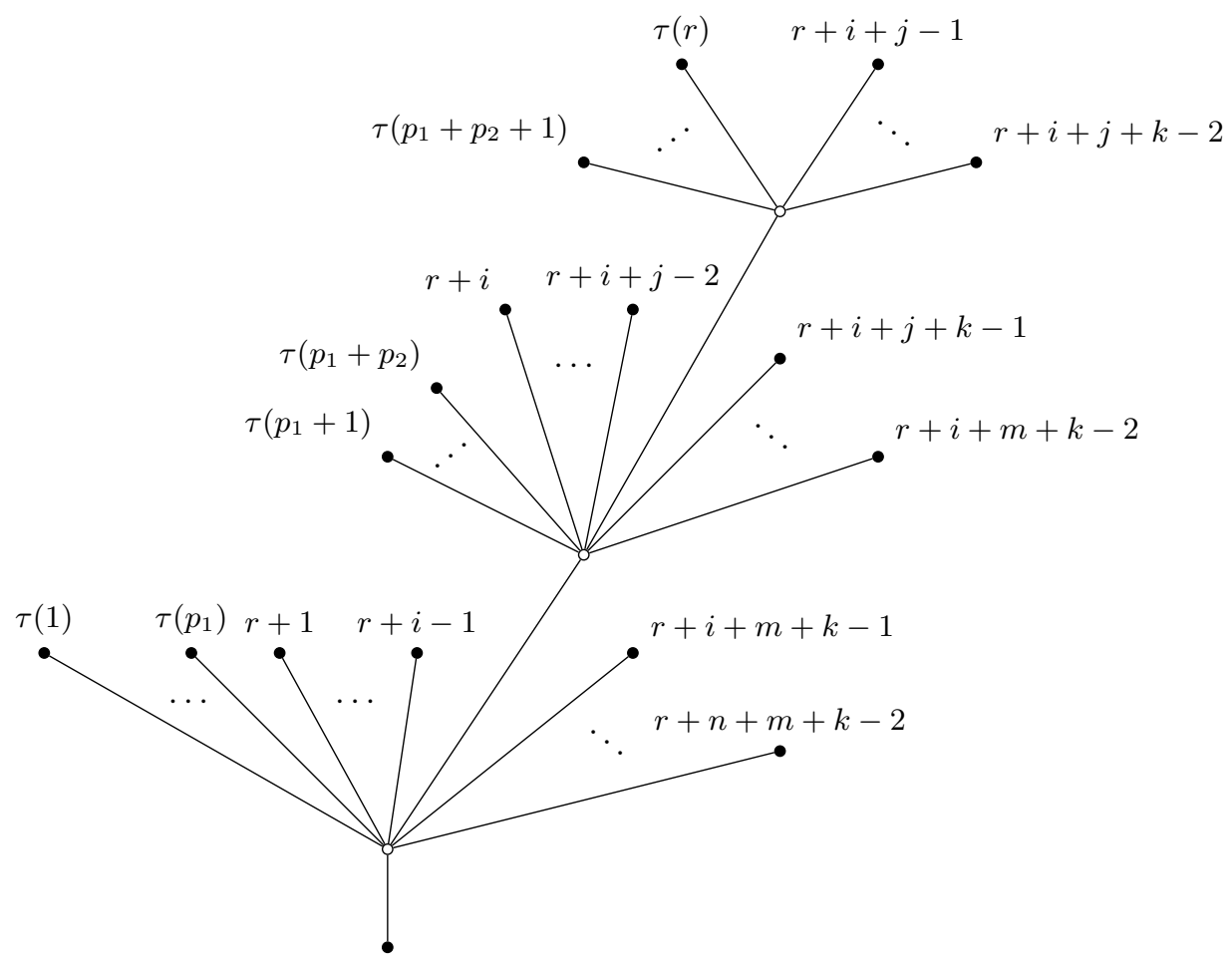

Fig. 3.2. Here $\tau$ is a $\left(p_{1}, p_{2}, p_{3}\right)$-shuffle and $r=p_{1}+p_{2}+p_{3}$

$$
\left(f \circ \circ_{i} g\right) \circ_{j+i-1} h=\sum_{p_{1}+p_{2}+p_{3}=r} \sum_{\tau \in \operatorname{Sh}_{p_{1}, p_{2}, p_{3}}} \mu_{\mathbf{t}_{\tau, i, j}}\left(f\left(1_{p_{1}}\right) \otimes g\left(1_{p_{2}}\right) \otimes h\left(1_{p_{3}}\right)\right),
$$

with $\mathbf{t}_{\tau, i, j}$ being the tree depicted on figure 3.2

We leave the verification of the remaining axioms of the operad structure for the reader.

Our next goal is to define an auxiliary action of the dg Lie algebra $\mathcal{L}_{\mathcal{O}}$ on the operad $\widetilde{\mathrm{Tw}} \mathcal{O}$. For a vector $f \in \widetilde{\mathrm{Tw}} \mathcal{O}(n)$ the action of $v \in \mathcal{L}_{\mathcal{O}} 3.5$ on $f$ is defined by the formula

$$
v \cdot f\left(1_{r}\right)=-(-1)^{|v||f|} \sum_{p=1}^{r} \sum_{\sigma \in \operatorname{Sh}_{p, r-p}} \mu_{\mathbf{t}_{\sigma, p, r-p}}\left(f\left(1_{r-p+1}\right) \otimes v\left(1_{p}^{\mathfrak{c}}\right)\right),
$$

where $1_{p}^{\mathfrak{c}}$ is the generator $\mathbf{s}^{2-2 p} 1 \in \Lambda^{2} \operatorname{coCom}(p) \cong \mathbf{s}^{2-2 p} \mathbb{K}$ and the tree $\mathbf{t}_{\sigma, p, r-p}$ is depicted on figure 3.3 .

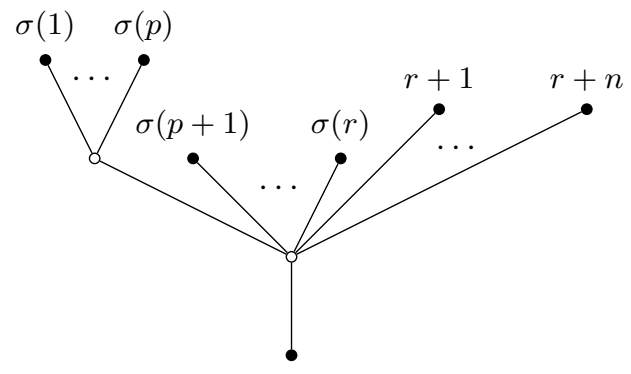

Fig. 3.3. Here $\sigma$ is a $(p, r-p)$-shuffle

We claim that 
Proposition 3.2. Formula 3.13 defines an action of the $d g$ Lie algebra $\mathcal{L}_{\mathcal{O}}$ (3.5) on the operad $\widetilde{\mathrm{Tw}} \mathcal{O}$.

Proof. A simple degree bookkeeping shows that the degree of $v \cdot f$ is $|v|+|f|$.

Then we need to check that for two homogeneous vectors $v, w \in \mathcal{L}_{\mathcal{O}}$ we have

$$
[v, w] \cdot f\left(1_{r}\right)=(v \cdot(w \cdot f))\left(1_{r}\right)-(-1)^{|v||w|}(w \cdot(v \cdot f))\left(1_{r}\right)
$$

Using the definition of the operation - and the associativity axiom for the operad structure on $\mathcal{O}$ we get

$$
\begin{aligned}
& (v \cdot(w \cdot f))\left(1_{r}\right)-(-1)^{|v||w|}(w \cdot(v \cdot f))\left(1_{r}\right)= \\
& (-1)^{|f|(|v|+|w|)+|v||w|} \sum_{p \geq 1 q \geq 0} \sum_{\tau \in \operatorname{Sh}_{p, q, r-p-q}} \mu_{\mathbf{t}_{\tau}^{p, q}}\left(f\left(1_{r-p-q+1}\right) \otimes w\left(1_{q+1}^{\mathfrak{c}}\right) \otimes v\left(1_{p}^{\mathfrak{c}}\right)\right) \\
& +(-1)^{|f|(|v|+|w|)+|v||w|} \sum_{p, q \geq 1} \sum_{\tau \in \operatorname{Sh}_{p, q, r-p-q}} \mu_{\widetilde{\mathbf{t}}_{\tau}^{p, q}}\left(f\left(1_{r-p-q+2}\right) \otimes w\left(1_{q}^{\mathfrak{c}}\right) \otimes v\left(1_{p}^{\mathfrak{c}}\right)\right) \\
& -(-1)^{|v||w|}(v \leftrightarrow w),
\end{aligned}
$$

where the trees $\mathbf{t}_{\tau}^{p, q}$ and $\widetilde{\mathbf{t}}_{\tau}^{p, q}$ are depicted on figures 3.4 and 3.5 , respectively.

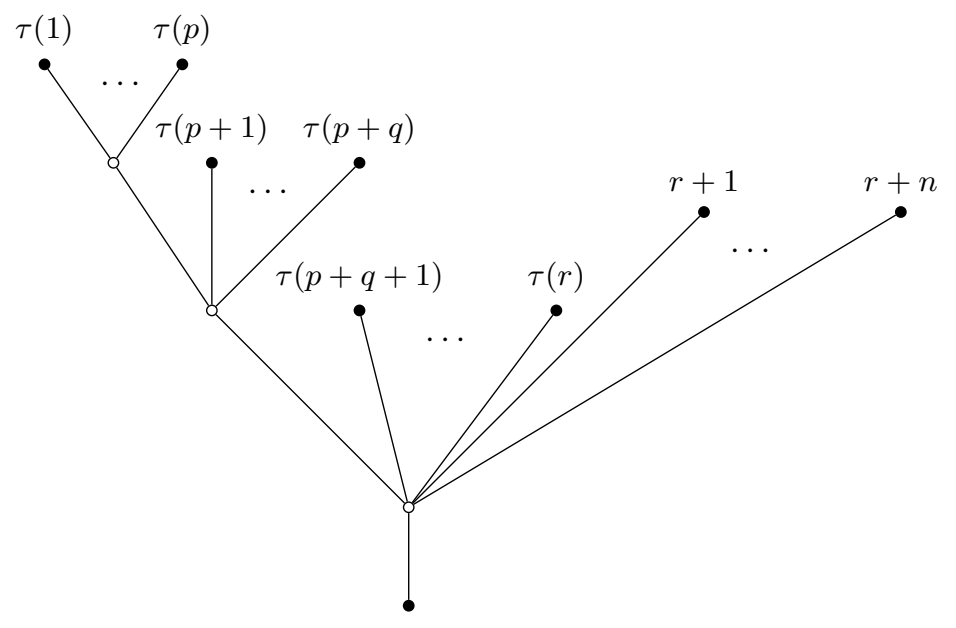

FIG. 3.4. The tree $\mathbf{t}_{\tau}^{p, q}$

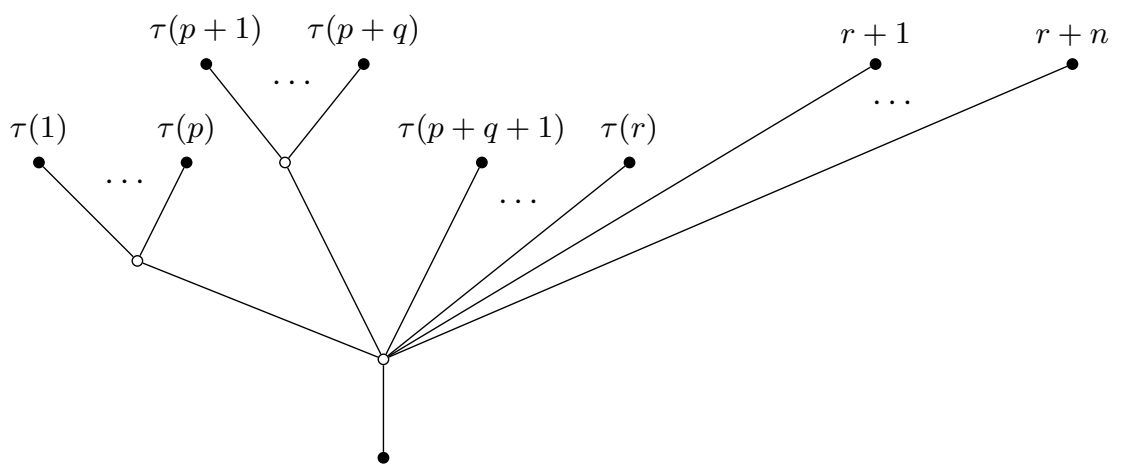

FIG. 3.5. The tree $\widetilde{\mathbf{t}}_{\tau}^{p, q}$ 
Since $f\left(1_{r-p-q+2}\right)$ is invariant with respect to the action of $S_{r-p-q+2}$ the sums involving $\mu_{\widetilde{\mathbf{t}}_{\tau}^{p, q}}$ cancel each other. Furthermore, it is not hard to see that the sums involving $\mu_{\mathbf{t}_{\tau}^{p, q}}$ form the expression

$$
[v, w] \cdot f\left(1_{r}\right) .
$$

Thus equation (3.14) follows. It remains to check that the operation $f \mapsto v \cdot f$ is an operadic derivation and we leave this step as an exercise for the reader.

3.1. The action of $\mathcal{L}_{\mathcal{O}}$ on $\widetilde{\mathrm{Tw}} \mathcal{O}$. Let us view $\widetilde{\mathrm{Tw}} \mathcal{O}(1)$ as the Lie algebra with the bracket being commutator.

We have an obvious degree zero map

$$
\kappa: \mathcal{L}_{\mathcal{O}} \rightarrow \widetilde{\mathrm{Tw}} \mathcal{O}(1)
$$

defined by the formula:

$$
\kappa(v)\left(1_{r}\right)=v\left(1_{r+1}^{\mathfrak{c}}\right) .
$$

where, as above, $1_{r}$ is the generator $\mathbf{s}^{-2 r} 1 \in \mathbf{s}^{-2 r} \mathbb{K}$ and $1_{r}^{\mathfrak{c}}$ is the generator $\mathbf{s}^{2-2 r} 1 \in \Lambda^{2} \operatorname{coCom}(r) \cong \mathbf{s}^{2-2 r} \mathbb{K}$.

We have the following proposition.

Proposition 3.3. Let us form the semi-direct product $\mathcal{L}_{\mathcal{O}} \ltimes \widetilde{\mathrm{Tw}} \mathcal{O}(1)$ of the $d g$ Lie algebras $\mathcal{L}_{\mathcal{O}}$ and $\widetilde{\mathrm{Tw}} \mathcal{O}(1)$ using the action of $\mathcal{L}_{\mathcal{O}}$ on $\widetilde{\mathrm{Tw}} \mathcal{O}$ defined in Proposition 3.2. Then the formula

$$
\Theta(v)=v+\kappa(v)
$$

defines a Lie algebra homomorphism

$$
\Theta: \mathcal{L}_{\mathcal{O}} \rightarrow \mathcal{L}_{\mathcal{O}} \ltimes \widetilde{\mathrm{Tw}} \mathcal{O}(1)
$$

Proof. First, let us prove that for every pair of homogeneous vectors $v, w \in \mathcal{L}_{\mathcal{O}}$ we have

$$
\kappa([v, w])=[\kappa(v), \kappa(w)]+v \cdot \kappa(w)-(-1)^{|v||w|} w \cdot \kappa(v) .
$$

Indeed, unfolding the definition of $\kappa$ we get ${ }^{8}$

$$
\begin{gathered}
\kappa([v, w])\left(1_{r}\right)=\sum_{p=1}^{r} \sum_{\tau \in \operatorname{Sh}_{p, r-p}} v_{r-p+2}\left(w_{p}(\tau(1), \ldots, \tau(p)), \tau(p+1), \ldots, \tau(r), r+1\right) \\
+\sum_{p=0}^{r} \sum_{\tau \in \operatorname{Sh}_{p, r-p}} v_{r-p+1}\left(w_{p+1}(\tau(1), \ldots, \tau(p), r+1), \tau(p+1), \ldots, \tau(r)\right) \\
-(-1)^{|v||w|}(v \leftrightarrow w),
\end{gathered}
$$

where $v_{t}=v\left(1_{t}\right)$ and $w_{t}=w\left(1_{t}\right)$. The first sum in (3.19) equals

$$
-(-1)^{|v||w|}(w \cdot \kappa(v))\left(1_{r}\right) .
$$

Furthermore, since $v\left(1_{t}\right)$ is invariant under the action of $S_{t}$, we see that the second sum in 3.19 equals

$$
\left(\kappa(v) \circ_{1} \kappa(w)\right)\left(1_{r}\right) .
$$

Thus equation (3.18) holds. Now, using (3.18), it is easy to see that

$$
[v+\kappa(v), w+\kappa(w)]=[v, w]+v \cdot \kappa(w)-(-1)^{|v||w|} w \cdot \kappa(v)+[\kappa(v), \kappa(w)]=
$$

\footnotetext{
${ }^{8}$ Here we use the notation for operadic multiplications 2.13 .
} 


$$
=[v, w]+\kappa([v, w])
$$

and the statement of the proposition follows.

The following corollaries are immediate consequences of Propositions 2.1 and 3.3

Corollary 3.4. For $v \in \mathcal{L}_{\mathcal{O}}$ and $f \in \widetilde{\mathrm{Tw}} \mathcal{O}(n)$ the formula

$$
f \rightarrow v \cdot f+\delta_{\kappa(v)}(f)
$$

defines an action of the Lie algebra $\mathcal{L}_{\mathcal{O}}$ on the operad $\widetilde{\mathrm{Tw}} \mathcal{O}$.

Corollary 3.5. For every Maurer-Cartan element $\varphi \in \mathcal{L}_{\mathcal{O}}$, the sum

$$
\varphi+\kappa(\varphi)
$$

is a Maurer-Cartan element of the Lie algebra $\mathcal{L}_{\mathcal{O}} \ltimes \widetilde{\mathrm{Tw}} \mathcal{O}(1)$.

We finally give the definition of the operad $\operatorname{Tw} \mathcal{O}$.

Definition 3.6. Let $\mathcal{O}$ be an operad in $\mathrm{Ch}_{\mathbb{K}}$ and $\varphi$ be a Maurer-Cartan element in $\mathcal{L}_{\mathcal{O}}$ 3.5 corresponding to an operad morphism $\widehat{\varphi}(3.1)$. Let us also denote by $\partial^{\mathcal{O}}$ the differential on $\widetilde{\mathrm{Tw}} \mathcal{O}$ coming from the one on $\mathcal{O}$. We define the operad $\operatorname{Tw} \mathcal{O}$ in $\mathrm{Ch}_{\mathbb{K}}$ by declaring that $\operatorname{Tw} \mathcal{O}=\widetilde{\mathrm{Tw}} \mathcal{O}$ as operads in grVect $_{\mathbb{K}}$ and letting

$$
\partial^{\mathrm{Tw}}=\partial^{\mathcal{O}}+\varphi \cdot+\delta_{\kappa(\varphi)}
$$

be the differential on $\operatorname{Tw} \mathcal{O}$.

Corollaries 3.4 and 3.5 imply that $\partial^{\mathrm{Tw}}$ is indeed a differential on $\mathrm{Tw} \mathcal{O}$.

Remark 3.7. It is easy to see that, if $\mathcal{O}(0)=\mathbf{0}$ then the cochain complexes $\mathbf{s}^{-2} \mathrm{Tw} \mathcal{O}(0)$ and $\mathcal{L}_{\mathcal{O}}$ (3.5) are tautologically isomorphic.

3.2. Algebras over Tw $\mathcal{O}$. Let us assume that $V$ is an algebra over $\mathcal{O}$ equipped with a complete decreasing filtration (3.2). We also assume that the $\mathcal{O}$-algebra structure on $V$ is compatible with this filtration.

Given a Maurer-Cartan element $\alpha \in \mathcal{F}_{1} V$, the formula

$$
\partial^{\alpha}(v)=\partial(v)+\sum_{r=1}^{\infty} \frac{1}{r !} \varphi\left(1_{r+1}^{\mathfrak{c}}\right)(\alpha, \ldots, \alpha, v)
$$

defines a new (twisted) differential on $V$. We will denote by $V^{\alpha}$ the cochain complex $V$ with this new differential. In this setting we have the following theorem:

Theorem 3.8. If $V^{\alpha}$ is the cochain complex obtained from $V$ via twisting the differential by the MaurerCartan element $\alpha$ then the formula

$$
\begin{gathered}
f\left(v_{1}, \ldots, v_{n}\right)=\sum_{r=0}^{\infty} \frac{1}{r !} f\left(1_{r}\right)\left(\alpha, \ldots, \alpha, v_{1}, \ldots, v_{n}\right) \\
f \in \operatorname{Tw} \mathcal{O}(n), \quad v_{i} \in V
\end{gathered}
$$

defines a Tw $\mathcal{O}$-algebra structure on $V^{\alpha}$. 
Proof. Let $f \in \operatorname{Tw} \mathcal{O}(n), g \in \operatorname{Tw} \mathcal{O}(k)$,

$$
f_{r}:=f\left(1_{r}\right) \in(\mathcal{O}(r+n))^{S_{r}}, \quad \text { and } \quad g_{r}=g\left(1_{r}\right) \in(\mathcal{O}(r+k))^{S_{r}} .
$$

Our first goal is to verify that

$$
\begin{gathered}
(-1)^{|g|\left(\left|v_{i}\right|+\cdots+\left|v_{i-1}\right|\right)} f\left(v_{1}, \ldots, v_{i-1}, g\left(v_{i}, \ldots, v_{i+k-1}\right), v_{i+k}, \ldots, v_{n+k-1}\right) \\
=f \circ_{i} g\left(v_{1}, \ldots, v_{n+k-1}\right) .
\end{gathered}
$$

The left hand side of 3.24 can be rewritten as

$$
\begin{gathered}
(-1)^{|g|\left(\left|v_{i}\right|+\cdots+\left|v_{i-1}\right|\right)} f\left(v_{1}, \ldots, v_{i-1}, g\left(v_{i}, \ldots, v_{i+k-1}\right), v_{i+k}, \ldots, v_{n+k-1}\right)= \\
\sum_{p, q \geq 0} \frac{(-1)^{|g|\left(\left|v_{i}\right|+\cdots+\left|v_{i-1}\right|\right)}}{p ! q !} f_{p}\left(\alpha, \ldots, \alpha, v_{1}, \ldots, v_{i-1}, g_{q}\left(\alpha, \ldots, \alpha, v_{i}, \ldots, v_{i+k-1}\right), v_{i+k}, \ldots, v_{n+k-1}\right) .
\end{gathered}
$$

Using the obvious combinatorial identity

$$
\left|\mathrm{Sh}_{p, q}\right|=\frac{(p+q) !}{p ! q !}
$$

we rewrite the left hand side of $(3.24)$ further

$$
\begin{gathered}
\text { L.H.S. of } \underline{3.24}= \\
\sum_{p, q \geq 0} \frac{(-1)^{|g|\left(\left|v_{i}\right|+\cdots+\left|v_{i-1}\right|\right)}}{(p+q) !}\left|\operatorname{Sh}_{p, q}\right| f_{p}\left(\alpha, \ldots, \alpha, v_{1}, \ldots, v_{i-1}, g_{q}\left(\alpha, \ldots, \alpha, v_{i}, \ldots, v_{i+k-1}\right), v_{i+k}, \ldots, v_{n+k-1}\right)= \\
\sum_{r=0}^{\infty} \frac{1}{r !} \sum_{p=0}^{r} \sum_{\sigma \in \operatorname{Sh}_{p, r-p}} \sigma \circ \varrho_{r, p, i}\left(f_{p} \circ_{p+i} g_{r-p}\right)(\underbrace{\alpha, \ldots, \alpha}_{r \text { arguments }}, v_{1}, \ldots, v_{n+k-1}),
\end{gathered}
$$

where $\varrho_{r, p, i}$ is the following permutation in $S_{r+i-1}$

$$
\varrho_{r, p, i}=\left(\begin{array}{cccccc}
p+1 & \ldots & p+i-1 & p+i & \ldots & r+i-1 \\
r+1 & \ldots & r+i-1 & p+1 & \ldots & r
\end{array}\right) .
$$

Thus, using (3.11), we get

$$
\text { L.H.S. of } 3.24=f \circ_{i} g\left(v_{1}, \ldots, v_{n+k-1}\right)
$$

and equation (3.24 holds.

Next, we need to show that

$$
\begin{aligned}
\partial^{\mathrm{Tw}}(f)\left(v_{1}, \ldots, v_{n}\right)=\partial^{\alpha} f\left(v_{1}, \ldots, v_{n}\right) & \\
& -(-1)^{|f|} \sum_{i=1}^{n}(-1)^{\left|v_{i}\right|+\cdots+\left|v_{i-1}\right|} f\left(v_{1}, \ldots, v_{i-1}, \partial^{\alpha}\left(v_{i}\right), v_{i+1}, \ldots, v_{n}\right) .
\end{aligned}
$$

The right hand side of (3.27) can be rewritten as

$$
\begin{aligned}
\text { R.H.S. of } & (3.27)=\sum_{p \geq 0} \frac{1}{p !} \partial f_{p}\left(\alpha, \ldots, \alpha, v_{1}, \ldots, v_{n}\right)+\sum_{p \geq 0, q \geq 1} \frac{1}{p ! q !} \varphi_{q}\left(\alpha, \ldots, \alpha, f_{p}\left(\alpha, \ldots, \alpha, v_{1}, \ldots, v_{n}\right)\right) \\
& -(-1)^{|f|} \sum_{i=1}^{n} \sum_{p \geq 0} \frac{(-1)^{\left|v_{i}\right|+\cdots+\left|v_{i-1}\right|}}{p !} f_{p}\left(\alpha, \ldots, \alpha, v_{1}, \ldots, v_{i-1}, \partial\left(v_{i}\right), v_{i+1}, \ldots, v_{n}\right) \\
& -(-1)^{|f|} \sum_{i=1}^{n} \sum_{p \geq 0, q \geq 1} \frac{(-1)^{\left|v_{i}\right|+\cdots+\left|v_{i-1}\right|}}{p ! q !} f_{p}\left(\alpha, \ldots, \alpha, v_{1}, \ldots, v_{i-1}, \varphi_{q}\left(\alpha, \ldots, \alpha, v_{i}\right), v_{i+1}, \ldots, v_{n}\right),
\end{aligned}
$$

where $f_{p}=f\left(1_{p}\right)$ and $\varphi_{q}=\varphi\left(1_{q}^{\mathfrak{c}}\right)$. 
Let us now add to and subtract from the right hand side of 3.27 the sum

$$
-(-1)^{|f|} \sum_{p \geq 0} \frac{1}{p !} f_{p+1}\left(\partial \alpha, \alpha, \ldots, \alpha, v_{1}, \ldots, v_{n}\right) .
$$

Using the symmetry of $f_{p}=f\left(1_{p}\right)$ with respect to the action of the subgroup $S_{p} \subset S_{p+n}$, we get

$$
\begin{aligned}
& \text { R.H.S. of } 3.27= \\
& \sum_{p \geq 0} \frac{1}{p !} \partial f_{p}\left(\alpha, \ldots, \alpha, v_{1}, \ldots, v_{n}\right)-(-1)^{|f|} \sum_{p \geq 0} \frac{1}{p !} f_{p+1}\left(\partial \alpha, \alpha, \ldots, \alpha, v_{1}, \ldots, v_{n}\right) \\
& -(-1)^{|f|} \sum_{i=1}^{n} \sum_{p \geq 0} \frac{(-1)^{\left|v_{i}\right|+\cdots+\left|v_{i-1}\right|}}{p !} f_{p}\left(\alpha, \ldots, \alpha, v_{1}, \ldots, v_{i-1}, \partial\left(v_{i}\right), v_{i+1}, \ldots, v_{n}\right) \\
& +(-1)^{|f|} \sum_{p \geq 0} \frac{1}{p !} f_{p+1}\left(\partial \alpha, \alpha, \ldots, \alpha, v_{1}, \ldots, v_{n}\right) \\
& +\sum_{p \geq 0, q \geq 1} \frac{1}{p ! q !} \varphi_{q}\left(\alpha, \ldots, \alpha, f_{p}\left(\alpha, \ldots, \alpha, v_{1}, \ldots, v_{n}\right)\right) \\
& -(-1)^{|f|} \sum_{i=1}^{n} \sum_{p \geq 0, q \geq 1} \frac{(-1)^{\left|v_{i}\right|+\cdots+\left|v_{i-1}\right|}}{p ! q !} f_{p}\left(\alpha, \ldots, \alpha, v_{1}, \ldots, v_{i-1}, \varphi_{q}\left(\alpha, \ldots, \alpha, v_{i}\right), v_{i+1}, \ldots, v_{n}\right)= \\
& \left(\partial^{\mathcal{O}} f\right)\left(v_{1}, \ldots, v_{n}\right) \\
& +(-1)^{|f|} \sum_{p \geq 0} \frac{1}{p !} f_{p+1}\left(\partial \alpha, \alpha, \ldots, \alpha, v_{1}, \ldots, v_{n}\right) \\
& +\sum_{p \geq 0, q \geq 1} \frac{1}{p ! q !} \varphi_{q}\left(\alpha, \ldots, \alpha, f_{p}\left(\alpha, \ldots, \alpha, v_{1}, \ldots, v_{n}\right)\right) \\
& -(-1)^{|f|} \sum_{i=1}^{n} \sum_{p \geq 0, q \geq 1} \frac{(-1)^{\left|v_{i}\right|+\cdots+\left|v_{i-1}\right|}}{p ! q !} f_{p}\left(\alpha, \ldots, \alpha, v_{1}, \ldots, v_{i-1}, \varphi_{q}\left(\alpha, \ldots, \alpha, v_{i}\right), v_{i+1}, \ldots, v_{n}\right) .
\end{aligned}
$$

Due to the Maurer-Cartan equation for $\alpha$

$$
\partial(\alpha)+\frac{1}{q !} \varphi_{q}(\alpha, \alpha, \ldots, \alpha)=0
$$

we have

$$
\begin{aligned}
&+(-1)^{|f|} \sum_{p \geq 0} \frac{1}{p !} f_{p+1}\left(\partial \alpha, \alpha, \ldots, \alpha, v_{1}, \ldots, v_{n}\right)= \\
&-(-1)^{|f|} \sum_{p \geq 0, q \geq 2} \frac{1}{p ! q !} f_{p+1}\left(\varphi_{q}(\alpha, \ldots, \alpha), \alpha, \ldots, \alpha, v_{1}, \ldots, v_{n}\right) .
\end{aligned}
$$

Hence, using combinatorial formula 3.25, we get

R.H.S. of 3.27$)=\left(\partial^{\mathcal{O}} f\right)\left(v_{1}, \ldots, v_{n}\right)+(\varphi \cdot f)\left(v_{1}, \ldots, v_{n}\right)$

$$
\kappa(\varphi) \circ_{1} f\left(v_{1}, \ldots, v_{n}\right)-(-1)^{|f|} f \circ_{1} \kappa(\varphi)\left(v_{1}, \ldots, v_{n}\right) .
$$

Theorem 3.8 is proven. 
Let us now observe that the $\mathrm{dg}$ operad $\mathrm{Tw} \mathcal{O}$ is equipped with a complete descending filtration. Namely,

$$
\mathcal{F}_{k} \operatorname{Tw} \mathcal{O}(n)=\left\{f \in \operatorname{Tw} \mathcal{O}(n) \mid f\left(1_{r}\right)=0 \quad \forall r<k\right\}
$$

It is clear that the operad structure on $\mathrm{Tw} \mathcal{O}$ is compatible with this filtration. The endomorphism operad End $_{V}$ also carries a complete descending filtration since so does $V$. This observation motivates the following definition:

Definition 3.9. A filtered $\mathrm{Tw} \mathcal{O}$-algebra is a cochain complex $V$ equipped with a complete descending filtration for which the operad map

$$
\mathrm{Tw} \mathcal{O} \rightarrow \mathrm{End}_{V}
$$

is compatible with the filtrations. We denote by $\operatorname{Alg}_{\mathrm{Tw}} \mathcal{O}$ filtr the category of filtered $\mathrm{Tw} \mathcal{O}$-algebras.

It is easy to see that the $\mathrm{Tw} \mathcal{O}$-algebra $V^{\alpha}$ from Theorem 3.8 is a filtered $\mathrm{Tw} \mathcal{O}$-algebra in the sense of this definition. The next theorem provides us with an equivalent description of the category of filtered Tw $\mathcal{O}$-algebras.

Theorem 3.10. Let $\mathrm{Alg}_{\mathcal{O}}^{\mathrm{MC}}$ be the category of pairs $(V, \alpha)$, where $V$ is an $\mathcal{O}$-algebra equipped with a complete descending filtration as in (3.2) and $\alpha \in \mathcal{F}_{1} V$ is a Maurer-Cartan element. Morphisms between pairs (V, $\alpha$ ) and $\left(V^{\prime}, \alpha^{\prime}\right)$ are morphisms of filtered $\mathcal{O}$-algebras $f: V \rightarrow V^{\prime}$ which satisfy the condition $f(\alpha)=\alpha^{\prime}$. The category $\mathrm{Alg}_{\mathrm{Tw} \mathcal{O}}^{\mathrm{filtr}}$ of filtered $\mathrm{Tw} \mathcal{O}$-algebras is isomorphic to the category $\mathrm{Alg}_{\mathcal{O}}^{\mathrm{MC}}$.

Proof. Theorem 3.8 yields a functor

$$
\mathfrak{F}: \operatorname{Alg}_{\mathcal{O}}^{\mathrm{MC}} \rightarrow \operatorname{Alg}_{\mathrm{Tw} \mathcal{O}}^{\mathrm{filtr}}
$$

from the category $\mathrm{Alg}_{\mathcal{O}}^{\mathrm{MC}}$ to the category $\mathrm{Alg}_{\mathrm{Tw} \mathcal{O}}^{\mathrm{filtr}}$ of filtered $\mathrm{Tw} \mathcal{O}$-algebras. The functor $\mathfrak{F}$ assigns to a pair $(V, \alpha)$ the cochain complex $V^{\alpha}$ with the differential $\partial^{\alpha}(3.22$ and the Tw $\mathcal{O}$-algebra structure defined by equation (3.23).

To define a functor in the opposite direction we produce a degree 2 element $u^{\circ} \in \mathcal{F}_{1}$ Tw $\mathcal{O}(0)$ using the identity element $u_{\mathcal{O}} \in \mathcal{O}(1)$ :

$$
u^{\circ}\left(1_{r}\right):= \begin{cases}u_{\mathcal{O}} & \text { if } \quad r=1 \\ 0 & \text { otherwise. }\end{cases}
$$

Let, as above, $\varphi$ be the Maurer-Cartan element in $\operatorname{Conv}\left(\Lambda^{2}\right.$ coCom, $\left.\mathcal{O}\right)$ corresponding to the morphism $\hat{\varphi}: \Lambda$ Lie $_{\infty} \rightarrow \mathcal{O}$. Also, let $\varphi_{r}:=\varphi\left(1_{r}^{\mathfrak{c}}\right)$.

Since $S_{r}$ acts trivially on $\varphi_{r}$, and $\partial^{\mathcal{O}}\left(u_{\mathcal{O}}\right)=0$ we get

$$
\partial^{\mathrm{Tw}}\left(u^{\circ}\right)\left(1_{r}\right)=\sum_{\sigma \in \mathrm{Sh}_{r-1,1}} \sigma\left(\varphi_{r} \circ_{r} u_{\mathcal{O}}\right)-u_{\mathcal{O}} \circ_{1} \varphi_{r}=r \varphi_{r}-\varphi_{r} .
$$

Thus

$$
\partial^{\mathrm{Tw}}\left(u^{\circ}\right)\left(1_{r}\right)=(r-1) \varphi_{r}
$$

Let us now consider a filtered Tw $\mathcal{O}$-algebra $W$ with the differential $\partial_{W}$. The element $u^{\circ} \in \mathcal{F}_{1} \mathrm{Tw} \mathcal{O}(0)$ gives us a degree 2 vector in $W$. We denote this vector by $\alpha$ and observe that $\alpha \in \mathcal{F}_{1} W$ since the map $\mathrm{Tw} \mathcal{O} \rightarrow \mathrm{End}_{W}$ is compatible with the filtrations. 
Next, we remark that the formula

$$
\iota(\gamma)\left(1_{r}\right):=\left\{\begin{array}{ll}
\gamma & \text { if } r=0 \\
0 & \text { otherwise, }
\end{array} \quad \gamma \in \mathcal{O}(n)\right.
$$

defines an embedding

$$
\iota: \mathcal{O} \hookrightarrow \operatorname{Tw} \mathcal{O}
$$

of operads in the category grVect $_{\mathbb{K}}$.

Thus, using $\iota$ and the Tw $\mathcal{O}$-algebra structure on $W$, we get an $\mathcal{O}$-algebra structure on $W$. Since, in general, $\iota$ is not compatible with the differentials, this $\mathcal{O}$-algebra structure on $W$ is not compatible with the differential $\partial_{W}$ on $W$.

Let $r$ be a non-negative integer. Then, for every vector

$$
f_{r} \in(\mathcal{O}(r+n))^{S_{r}}
$$

the elements $f_{r}, \mathbf{s}^{2} f_{r}, \mathbf{s}^{4} f_{r}, \ldots, \mathbf{s}^{2 r} f_{r}$ may be viewed as vectors in Tw $\mathcal{O}(r+n)$, Tw $\mathcal{O}(r-1+n)$, Tw $\mathcal{O}(r-$ $2+n), \ldots$, Tw $\mathcal{O}(n)$, respectively. Namely,

$$
\mathbf{s}^{2 k} f_{r}\left(1_{p}\right):= \begin{cases}f_{r} & \text { if } p=k \\ 0 & \text { otherwise }\end{cases}
$$

Let us consider the vector $\mathbf{s}^{2 k} f_{r} \circ_{1} u^{\circ} \in \operatorname{Tw} \mathcal{O}(r-k-1+n)$ for $0 \leq k \leq r-1$. It is clear that for $p \neq k+1$

$$
\left(\mathbf{s}^{2 k} f_{r} \circ_{1} u^{\circ}\right)\left(1_{p}\right)=0 .
$$

For $p=k+1$ we use the $S_{r}$-invariance of $f_{r}$ and get

$$
\left(\mathbf{s}^{2 k} f_{r} \circ_{1} u^{\circ}\right)\left(1_{k+1}\right)=\sum_{\sigma \in \mathrm{Sh}_{k, 1}} \sigma\left(f_{r} \circ_{k+1} u_{\mathcal{O}}\right)=(k+1) f_{r} .
$$

Thus

$$
\mathbf{s}^{2 k} f_{r} \circ_{1} u^{\circ}=(k+1) \mathbf{s}^{2 k+2} f_{r} .
$$

Applying identity $3.35 r$ times we get

$$
\left(\ldots \left((f_{r} \underbrace{\left.\left.\left.\circ_{1} u^{\circ}\right) \circ_{1} u^{\circ}\right) \cdots \circ_{1} u^{\circ}\right)}_{r \text { times }}=r ! \mathrm{s}^{2 r} f_{r} .\right.\right.
$$

Combining this observation with the fact that the filtration on $W$ is complete, we conclude that for every vector $f \in \operatorname{Tw} \mathcal{O}(n)$ with $f_{r}=f\left(1_{r}\right) \in(\mathcal{O}(r+n))^{S_{r}}$ we have

$$
f\left(w_{1}, w_{2}, \ldots, w_{n}\right)=\sum_{r=0}^{\infty} \frac{1}{r !} \iota\left(f_{r}\right)\left(\alpha, \ldots, \alpha, w_{1}, w_{2}, \ldots, w_{n}\right), \quad w_{i} \in W
$$

where $\iota$ is the map of operads $\mathcal{O} \rightarrow \mathrm{Tw} \mathcal{O}$ in grVect $_{\mathbb{K}}$ defined in 3.32 .

Let us denote by $\partial$ the degree 1 operation

$$
\partial: W \rightarrow W
$$

defined by the equation

$$
\partial(w):=\partial_{W}(w)-\sum_{r=1}^{\infty} \frac{1}{r !} \iota\left(\varphi_{r+1}\right)(\alpha, \ldots, \alpha, w),
$$


where, as above, $\varphi_{q}=\varphi\left(1_{q}^{\mathfrak{c}}\right)$.

To prove the identity $\partial^{2}=0$, we observe that, due to equation (3.37),

$$
\partial(w)=\partial_{W}(w)-\kappa(\varphi)(w) .
$$

Hence

$$
\begin{gathered}
\partial^{2}(w)=-\partial_{W}(\kappa(\varphi)(w))-\kappa(\varphi)\left(\partial_{W}(w)\right)+\left(\kappa(\varphi) \circ_{1} \kappa(\varphi)\right)(w)= \\
-\left(\partial^{\mathrm{Tw}} \kappa(\varphi)\right)(w)+\left(\kappa(\varphi) \circ_{1} \kappa(\varphi)\right)(w)= \\
-\left(\partial^{\mathcal{O}} \kappa(\varphi)\right)(w)-(\varphi \cdot \kappa(\varphi))(w)-2\left(\kappa(\varphi) \circ_{1} \kappa(\varphi)\right)(w)+\left(\kappa(\varphi) \circ_{1} \kappa(\varphi)\right)(w)= \\
-\left(\partial^{\mathcal{O}} \kappa(\varphi)+\varphi \cdot \kappa(\varphi)+\kappa(\varphi) \circ_{1} \kappa(\varphi)\right)(w) .
\end{gathered}
$$

On the other hand, the vector $\partial^{\mathcal{O}} \kappa(\varphi)+\varphi \cdot \kappa(\varphi)+\kappa(\varphi) \circ_{1} \kappa(\varphi) \in \mathrm{Tw} \mathcal{O}(1)$ is zero due to Corollary 3.5. Thus equation (3.38) (or equation (3.39) defines another differential on $W$.

Let us show that the differential $\partial$ is compatible with the $\mathcal{O}$-algebra structure given by $\iota(3.33)$.

Equation $(3.39)$ implies that for every $\gamma \in \mathcal{O}(n)$ and $w_{1}, w_{2}, \ldots, w_{n} \in W$, we have

$$
\begin{aligned}
& \partial\left(\iota(\gamma)\left(w_{1}, \ldots, w_{n}\right)\right)-\sum_{i=1}^{n}(-1)^{|\gamma|+\left|w_{1}\right|+\cdots+\left|w_{i-1}\right|} \iota(\gamma)\left(w_{1}, \ldots, w_{i-1}, \partial\left(w_{i}\right), w_{i+1}, \ldots, w_{n}\right) \\
& =\partial_{W}\left(\iota(\gamma)\left(w_{1}, \ldots, w_{n}\right)\right)-\sum_{i=1}^{n}(-1)^{|\gamma|+\left|w_{1}\right|+\cdots+\left|w_{i-1}\right|} \iota(\gamma)\left(w_{1}, \ldots, w_{i-1}, \partial_{W}\left(w_{i}\right), w_{i+1}, \ldots, w_{n}\right) \\
& \quad-\kappa(\varphi)\left(\iota(\gamma)\left(w_{1}, \ldots, w_{n}\right)\right) \\
& \quad+\sum_{i=1}^{n}(-1)^{|\gamma|+\left|w_{1}\right|+\cdots+\left|w_{i-1}\right|} \iota(\gamma)\left(w_{1}, \ldots, w_{i-1}, \kappa(\varphi)\left(w_{i}\right), w_{i+1}, \ldots, w_{n}\right) \\
& =\left(\partial^{\mathrm{Tw}} \iota(\gamma)\right)\left(w_{1}, \ldots, w_{n}\right)-\left(\kappa(\varphi) \circ_{1} \iota(\gamma)\right)\left(w_{1}, \ldots, w_{n}\right)+(-1)^{|\gamma|} \sum_{i=1}^{n}\left(\iota(\gamma) \circ_{1} \kappa(\varphi)\right)\left(w_{1}, \ldots, w_{n}\right) \\
& =\left(\iota\left(\partial^{\mathcal{O}} \gamma\right)\right)\left(w_{1}, \ldots, w_{n}\right),
\end{aligned}
$$

where, in the last step, we used the fact that $\iota(\gamma)\left(1_{r}\right)=0$ for all $r \geq 1$.

Thus the differential $\partial(3.38)$ is indeed compatible with the $\mathcal{O}$-algebra structure on $W$.

It remains to prove that the vector $\alpha \in W$ is a Maurer-Cartan element (with respect to the differential $\partial)$.

For this purpose we observe that, since the map $\mathrm{Tw} \mathcal{O} \rightarrow \mathrm{End}_{W}$ is compatible with the differentials, the vector $\partial^{\mathrm{Tw}} u^{\circ}$ maps to $\partial_{W}(\alpha)$ in $W$.

Therefore, combining equation 3.31 with equation (3.37) we get

$$
\partial_{W}(\alpha)=\sum_{r=2}^{\infty} \frac{r-1}{r !} \iota\left(\varphi_{r}\right)(\alpha, \ldots, \alpha)
$$

or equivalently

$$
\partial(\alpha)+\sum_{r=1}^{\infty} \frac{1}{r !} \iota\left(\varphi_{r+1}\right)(\alpha, \ldots, \alpha)=\sum_{r=2}^{\infty} \frac{r-1}{r !} \iota\left(\varphi_{r}\right)(\alpha, \ldots, \alpha) .
$$

Thus $\alpha$ indeed satisfies the Maurer-Cartan equation

$$
\partial(\alpha)+\sum_{r=2}^{\infty} \frac{1}{r !} \iota\left(\varphi_{r}\right)(\alpha, \ldots, \alpha)=0
$$


Combining the performed work, we conclude that the cochain complex $W$ with the differential (3.38) and the Maurer-Cartan element $\alpha$ is an object of the category $\operatorname{Alg}_{\mathcal{O}}^{\mathrm{MC}}$.

Equations (3.37) and (3.38) imply that the described construction, indeed, gives a (strict) inverse for the functor $\mathfrak{F}(3.29)$.

3.3. Example: The dg operad $\mathrm{Tw}_{\mathrm{w}} \Lambda \mathrm{Lie}_{\infty}$. In this subsection, we apply the twisting procedure to the pair $\left(\Lambda \mathrm{Lie}_{\infty}, \hat{\varphi}\right)$, where

$$
\hat{\varphi}=\mathrm{id}: \Lambda \mathrm{Lie}_{\infty} \rightarrow \Lambda \mathrm{Lie}_{\infty} .
$$

Let us recall that $1_{r}^{\mathfrak{c}}$ denotes the canonical generator $\mathbf{s}^{2-2 r} 1 \in \Lambda^{2} \operatorname{coCom}_{\circ}(r)$. Thus, the Maurer-Cartan element $\varphi$ corresponding to 3.41 is

$$
\varphi\left(1_{r}^{\mathfrak{c}}\right)=\mathbf{s} 1_{r}^{\mathfrak{c}}, \quad r \geq 2
$$

and the element $\kappa(\varphi) \in \mathrm{Tw} \Lambda \mathrm{Lie}_{\infty}(1)$ takes the form

$$
\kappa(\varphi)\left(1_{r}\right)=\left\{\begin{array}{l}
\mathbf{s} 1_{r+1}^{\mathfrak{c}} \quad \text { if } r \geq 1, \\
0 \quad \text { if } r=0 .
\end{array}\right.
$$

According to general formula (3.21), the differential $\partial^{\mathrm{Tw}}$ on $\mathrm{Tw} \Lambda \mathrm{Lie}_{\infty}$ is given by the equation

$$
\begin{gathered}
\partial^{\mathrm{Tw}}(f)\left(1_{r}\right)=\partial^{\mathrm{Cobar}}\left(f_{r}\right)-(-1)^{|f|} \sum_{p=2}^{r} \sum_{\sigma \in \mathrm{Sh}_{p, r-p}} \sigma\left(f_{r-p+1} \circ_{1}\left(\mathbf{s} 1_{p}^{\mathfrak{c}}\right)\right) \\
+\sum_{p=1}^{r} \sum_{\sigma \in \mathrm{Sh}_{p, r-p}} \sigma\left(\left(\mathbf{s} 1_{p+1}^{\mathfrak{c}}\right) \circ_{p+1} f_{r-p}\right)-(-1)^{|f|} \sum_{i=1}^{n} \sum_{p=1}^{r-1} \sum_{\sigma \in \operatorname{Sh}_{p, r-p}} \sigma \circ \varrho_{r, p, i}\left(f_{p} \circ_{p+i}\left(\mathbf{s} 1_{r-p+1}^{\mathfrak{c}}\right)\right) .
\end{gathered}
$$

where $f \in \mathrm{Tw} \Lambda \mathrm{Lie}_{\infty}(n), f_{r}=f\left(1_{r}\right)$ and $\varrho_{r, p, i}$ is the permutation of $S_{r+i-1}$ defined in 3.26 .

We will need the following lemma:

Lemma 3.11. If $\mathrm{Tw} \Lambda \mathrm{Lie}_{\infty}$ is the dg operad which is obtained from $\Lambda \mathrm{Lie}_{\infty}$ via applying the twisting procedure to the identity map (3.41) then the equation

$$
\mathfrak{T}\left(\mathbf{s} 1_{n}^{\mathfrak{c}}\right)\left(1_{r}\right)=\mathbf{s} 1_{r+n}^{\mathfrak{c}}
$$

defines a map of $d g$ operads

$$
\mathfrak{T}: \Lambda \mathrm{Lie}_{\infty} \rightarrow \mathrm{Tw} \Lambda \mathrm{Lie}_{\infty}
$$

Proof. According to 2.24

$$
\Lambda \mathrm{Lie}_{\infty}=\operatorname{Cobar}\left(\Lambda^{2} \text { coCom }\right) .
$$

Hence, due to Theorem 2.2, maps of dg operads from $\Lambda \mathrm{Lie}_{\infty}$ to $\mathrm{Tw} \Lambda \mathrm{Lie}_{\infty}$ are in bijection with Maurer-Cartan elements of the convolution Lie algebra

$$
\operatorname{Conv}\left(\Lambda^{2} \operatorname{coCom}_{\circ}, \operatorname{Tw} \Lambda \operatorname{Lie}_{\infty}\right)=\prod_{n \geq 2} \operatorname{Hom}_{S_{n}}\left(\mathbf{s}^{2-2 n} \mathbb{K}, \operatorname{Tw} \Lambda \operatorname{Lie}_{\infty}(n)\right) .
$$

Let us denote by $\alpha_{\mathfrak{T}}$ a vector in (3.47) defined by the equation:

$$
\alpha_{\mathfrak{T}}\left(1_{n}^{\mathfrak{c}}\right)\left(1_{r}\right)=\mathbf{s} 1_{r+n}^{\mathfrak{c}} .
$$

Since the vectors $1_{n}^{\mathfrak{c}}$ and $1_{n}$ carry degrees

$$
\left|1_{n}^{\mathfrak{c}}\right|=2-2 n, \quad\left|1_{n}\right|=-2 n,
$$


the vector $\alpha_{\mathfrak{T}}$ has degree 1 .

Using formula 3.44 for the differential $\partial^{\mathrm{Tw}}$ on $\mathrm{Tw} \Lambda$ Lie $_{\infty}$ we get (here $n \geq 2$ )

$$
\begin{aligned}
& \left(\partial^{\mathrm{Tw}} \alpha_{\mathfrak{T}}\left(1_{n}^{\mathfrak{c}}\right)\right)\left(1_{r}\right)=\partial^{\operatorname{Cobar}}\left(\mathbf{s} 1_{r+n}^{\mathfrak{c}}\right)+\sum_{p=2}^{r} \sum_{\sigma \in \operatorname{Sh}_{p, r-p}} \sigma\left(\mathbf{s} 1_{r-p+1+n}^{\mathfrak{c}} \circ_{1} \mathbf{s} 1_{p}^{\mathfrak{c}}\right)+ \\
& \sum_{p=1}^{r} \sum_{\sigma \in \mathrm{Sh}_{p, r-p}} \sigma\left(\mathbf{s} 1_{p+1}^{\mathfrak{c}} \circ_{p+1} \mathbf{s} 1_{r-p+n}^{\mathfrak{c}}\right)+\sum_{i=1}^{n} \sum_{p=1}^{r-1} \sum_{\sigma \in \operatorname{Sh}_{p, r-p}} \sigma \circ \varrho_{r, p, i}\left(\mathbf{s} 1_{p+n}^{\mathfrak{c}} \circ_{p+i} \mathbf{s} 1_{r-p+1}^{\mathfrak{c}}\right)
\end{aligned}
$$

where $\varrho_{r, p, i}$ is the permutation defined in 3.26 .

Unfolding $\partial^{\text {Cobar }}\left(\mathbf{s} 1_{r+n}^{\mathfrak{c}}\right)$, we get

$$
\begin{gathered}
\left(\partial^{\mathrm{Tw}} \alpha_{\mathfrak{T}}\left(1_{n}^{\mathfrak{c}}\right)\right)\left(1_{r}\right)=-\sum_{q=2}^{r+n-1} \sum_{\lambda \in \mathrm{Sh}_{q, r+n-q}} \lambda\left(\mathbf{s} 1_{r+n-q+1}^{\mathfrak{c}} \circ_{1} \mathbf{s} 1_{q}^{\mathfrak{c}}\right)+\sum_{p=2}^{r} \sum_{\sigma \in \mathrm{Sh}_{p, r-p}} \sigma\left(\mathbf{s} 1_{r-p+1+n}^{\mathfrak{c}} \circ_{1} \mathbf{s} 1_{p}^{\mathfrak{c}}\right)+ \\
\sum_{p=1}^{r} \sum_{\sigma \in \mathrm{Sh}_{p, r-p}} \sigma\left(\mathbf{s} 1_{p+1}^{\mathfrak{c}}{ }_{{ }_{p+1}} \mathbf{s} 1_{r-p+n}^{\mathfrak{c}}\right)+\sum_{i=1}^{n} \sum_{p=1}^{r-1} \sum_{\sigma \in \operatorname{Sh}_{p, r-p}} \sigma \circ \varrho_{r, p, i}\left(\mathbf{s} 1_{p+n}^{\mathfrak{c}} \circ_{p+i} \mathbf{s} 1_{r-p+1}^{\mathfrak{c}}\right)
\end{gathered}
$$

Next, using the definition of the binary operation 2.20 we get

$$
\begin{aligned}
\left(\alpha_{\mathfrak{T}} \bullet \alpha_{\mathfrak{T}}\left(1_{n}^{\mathfrak{c}}\right)\right)\left(1_{r}\right) & =\sum_{q=2}^{n-1} \sum_{\tau \in \mathrm{Sh}_{q, n-q}}\left(\tau\left(\alpha_{\mathfrak{T}}\left(1_{n-q+1}^{\mathfrak{c}}\right) \circ_{1} \alpha_{\mathfrak{T}}\left(1_{q}^{\mathfrak{c}}\right)\right)\right)\left(1_{r}\right) \\
& =\sum_{0 \leq p \leq r}^{2 \leq q \leq n-1} \sum_{\sigma \in \operatorname{Sh}_{p, r-p, q, n-q}} \sigma\left(\mathbf{s} 1_{n+p-q+1}^{\mathfrak{c}} \circ_{p+1} \mathbf{s} 1_{r-p+q}^{\mathfrak{c}}\right) .
\end{aligned}
$$

We observe that the second sum in the right hand side of 3.50 is obtained from

$$
\sum_{q=2}^{r+n-1} \sum_{\lambda \in \operatorname{Sh}_{q, r+n-q}} \lambda\left(\mathbf{s} 1_{r+n-q+1}^{\mathfrak{c}} \circ_{1} \mathbf{s} 1_{q}^{\mathfrak{c}}\right)
$$

by keeping only the terms for which

$$
\lambda(r+1)=r+1, \quad \lambda(r+2)=r+2, \quad \ldots, \quad \lambda(r+n)=r+n .
$$

Next, using the fact that $S_{m}$ acts trivially on the vector $1_{m}^{\mathfrak{c}}$, we see that the third sum in the right hand side of 3.50 is obtained from 3.52 by keeping only the terms for which $q \geq n$ and

$$
\lambda(q-n+1)=r+1, \quad \lambda(q-n+2)=r+2, \quad \ldots, \quad \lambda(q)=r+n .
$$

Similarly, the last sum in the right hand side of 3.50 is obtained from the sum 3.52 by keeping only the terms for which

$$
\lambda(q) \geq r+1 \quad \text { and } \quad \lambda(i) \leq r \quad \forall 1 \leq i \leq q-1
$$

Finally, using the invariance with respect to the action of the symmetric group once again, we see that $\left(\alpha_{\mathfrak{T}} \bullet \alpha_{\mathfrak{T}}\left(1_{n}^{\mathfrak{c}}\right)\right)\left(1_{r}\right)$ is obtained from the sum 3.52 by keeping only the terms for which

$$
\lambda(q-1) \geq r+1, \quad \text { and } \quad \lambda(r+n) \geq r+1
$$

Thus, the vector $\alpha_{\mathfrak{T}}$ satisfies the Maurer-Cartan equation

$$
\partial^{\mathrm{Tw}} \alpha_{\mathfrak{T}}+\alpha_{\mathfrak{T}} \bullet \alpha_{\mathfrak{T}}=0
$$


Since the morphism of dg operads

$$
\mathfrak{T}: \Lambda \mathrm{Lie}_{\infty} \rightarrow \mathrm{Tw} \Lambda \mathrm{Lie}_{\infty}
$$

corresponding to the Maurer-Cartan element $\alpha_{\mathfrak{T}}$ is defined by equation 3.45 , the lemma is proved.

Remark 3.12. Let $V$ be a $\Lambda$ Lie $_{\infty}$-algebra with a differential $\partial$ and the brackets

$$
\{,, \ldots,\}_{n}: V^{\otimes n} \rightarrow V .
$$

Let us assume that $V$ is equipped with a complete descending filtration (3.2) and $\alpha \in \mathcal{F}_{1} V$ is a Maurer-Cartan element. Theorem 3.8 and Lemma 3.11 imply that the formula

$$
\left\{v_{1}, \ldots, v_{n}\right\}_{n}^{\alpha}=\sum_{r=0}^{\infty} \frac{1}{r !}\{\underbrace{\alpha, \ldots, \alpha}_{r \text { times }}, v_{1}, \ldots, v_{n}\}_{r+n}
$$

defines a $\Lambda \mathrm{Lie}_{\infty}$-structure on $V$ with the differential given by equation 3.22 . We say that this latter $\Lambda$ Lie ${ }_{\infty}$ structure on $V$ is obtained from the former one via twisting by the Maurer-Cartan element $\alpha$.

3.4. A useful modification $\mathrm{Tw}^{\oplus} \mathcal{O}$. In practice the morphism 3.1 often comes from the map (of dg operads)

$$
\mathfrak{i}: \Lambda \text { Lie } \rightarrow \mathcal{O} \text {. }
$$

In this case, the above construction of twisting is well defined for the suboperad $\mathrm{Tw}^{\oplus}(\mathcal{O}) \subset \mathrm{Tw} \mathcal{O}$ with

$$
\mathrm{Tw}^{\oplus}(\mathcal{O})(n)=\bigoplus_{r \geq 0} \mathrm{~s}^{2 r}(\mathcal{O}(r+n))^{S_{r}} .
$$

It is not hard to see that the Maurer-Cartan element

$$
\varphi \in \operatorname{Conv}\left(\Lambda^{2} \operatorname{coCom}, \mathcal{O}\right)
$$

corresponding to the composition

$$
\mathfrak{i} \circ U_{\Lambda \text { Lie }}: \operatorname{Cobar}\left(\Lambda^{2} \operatorname{coCom}\right) \rightarrow \mathcal{O}
$$

is given by the formula:

$$
\varphi\left(1_{r}^{\mathfrak{c}}\right)=\left\{\begin{array}{l}
\mathfrak{i}\left(\left\{a_{1}, a_{2}\right\}\right) \quad \text { if } r=2 \\
0 \quad \text { otherwise }
\end{array}\right.
$$

Hence

$$
\mathcal{L}_{\mathcal{O}}^{\oplus}=\bigoplus_{r \geq 0} \mathbf{s}^{2 r-2}(\mathcal{O}(r))^{S_{r}}
$$

is a sub- dg Lie algebra of $\mathcal{L}_{\mathcal{O}}$ 3.5.

Specifying general formula 3.21 to this particular case, we see that the differential $\partial^{\mathrm{Tw}}$ on 3.55 is given by the equation:

$$
\begin{aligned}
\partial^{\mathrm{Tw}}(v)= & -(-1)^{|v|} \sum_{\sigma \in \mathrm{Sh}_{2, r-1}} \sigma\left(v \circ_{1} \mathfrak{i}\left(\left\{a_{1}, a_{2}\right\}\right)\right)+\sum_{\tau \in \mathrm{Sh}_{1, r}} \tau\left(\mathfrak{i}\left(\left\{a_{1}, a_{2}\right\}\right) \circ_{2} v\right) \\
& -(-1)^{|v|} \sum_{\tau^{\prime} \in \mathrm{Sh}_{r, 1}} \sum_{i=1}^{n} \tau^{\prime} \circ \varsigma_{r+1, r+i}\left(v \circ_{r+i} \mathfrak{i}\left(\left\{a_{1}, a_{2}\right\}\right)\right)
\end{aligned}
$$

where

$$
v \in \mathbf{s}^{2 r}(\mathcal{O}(r+n))^{S_{r}}
$$


and $\varsigma_{r+1, r+i}$ is the cycle $(r+1, r+2, \ldots, r+i)$.

Remark 3.13. We should remark that, when we apply elementary insertions in the right hand side of (3.58), we view $v$ and $\mathfrak{i}\left(\left\{a_{1}, a_{2}\right\}\right)$ as vectors in $\mathcal{O}(r+n)$ and $\mathcal{O}(2)$ respectively. The resulting sum in the right hand side of 3.58 is viewed as a vector in $\operatorname{Tw} \mathcal{O}(n)$.

3.5. More general version of twisting. The reader may object that the version of twisting we have presented so far does not even cover the most classical case of the operad $\mathcal{O}=$ Lie, since in (3.1) we required a map $\Lambda \mathrm{Lie}_{\infty} \rightarrow \mathcal{O}$. However, this is easily repaired:

Let $k$ be an integer and let

$$
\Lambda^{k} \operatorname{Lie}_{\infty} \rightarrow \mathcal{O}
$$

be an operad map. Then, by functoriality of $\Lambda$, there is an operad map

$$
\Lambda \mathrm{Lie}_{\infty} \rightarrow \Lambda^{1-k} \mathcal{O}
$$

We then define:

$$
\operatorname{Tw} \mathcal{O}:=\Lambda^{k-1} \operatorname{Tw}\left(\Lambda^{1-k} \mathcal{O}\right) .
$$

In this paper we will always assume $k=1$ since (i) this is the relevant case for the Deligne conjecture and (ii) the signs are significantly simpler for odd $k$. One should however be aware that all important statements may be transcribed to the arbitrary- $k$ case by application of the functor $\Lambda$.

\section{Categorial properties of twisting}

4.1. Tw as a comonad. The goal of this section is to show that the operation $\mathrm{Tw}$ defines a comonad on the under-category $\Lambda \mathrm{Lie}_{\infty} \downarrow$ Operads, where Operads is the category of dg operads. Recall that the under-category $\Lambda$ Lie $_{\infty} \downarrow$ Operads is the category of arrows $\Lambda$ Lie $_{\infty} \rightarrow \mathcal{O}$ in Operads, with morphisms the commutative diagrams

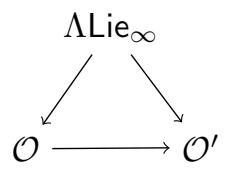

4.1.1. Tw is an endofunctor. Consider arrows $\Lambda \mathrm{Lie}_{\infty} \rightarrow \mathcal{O} \stackrel{f}{\rightarrow} \mathcal{O}^{\prime}$ in the category Operads. Twisting $\mathcal{O}$ and $\mathcal{O}^{\prime}$, we obtain new dg operads with

$$
\begin{aligned}
\operatorname{Tw} \mathcal{O}(n) & =\prod_{r \geq 0} \operatorname{Hom}_{S_{r}}\left(\mathbf{s}^{-2 r} \mathbb{K}, \mathcal{O}(r+n)\right) \\
\operatorname{Tw} \mathcal{O}^{\prime}(n) & =\prod_{r \geq 0} \operatorname{Hom}_{S_{r}}\left(\mathbf{s}^{-2 r} \mathbb{K}, \mathcal{O}^{\prime}(r+n)\right) .
\end{aligned}
$$

By composing morphisms on the right with (components of) the map $f: \mathcal{O} \rightarrow \mathcal{O}^{\prime}$ we obtain a map (of collections)

$$
\operatorname{Tw} f: \operatorname{Tw} \mathcal{O} \rightarrow \operatorname{Tw} \mathcal{O}^{\prime} .
$$

Lemma 4.1. The above map $\operatorname{Tw} f: \operatorname{Tw} \mathcal{O} \rightarrow \operatorname{Tw} \mathcal{O}^{\prime}$ is a map of $d g$ operads. Furthermore, if

$$
\Lambda \mathrm{Lie}_{\infty} \rightarrow \mathcal{O} \stackrel{f}{\rightarrow} \mathcal{O}^{\prime} \stackrel{g}{\rightarrow} \mathcal{O}^{\prime \prime}
$$

are maps of $d g$ operads, then $\operatorname{Tw}(f \circ g)=(\operatorname{Tw} f) \circ(\operatorname{Tw} g)$. 
Proof. It is clear since we only used natural operations (i.e., the operad structure) in defining Tw $\mathcal{O}$.

Applying Tw to $\hat{\varphi}: \Lambda \operatorname{Lie}_{\infty} \rightarrow \mathcal{O}$, we obtain a morphism of dg operads

$$
\operatorname{Tw}(\hat{\varphi}): \operatorname{Tw} \Lambda \operatorname{Lie}_{\infty} \rightarrow \operatorname{Tw} \mathcal{O} .
$$

Composing 4.1 with the canonical map of dg operads

$$
\mathfrak{T}: \Lambda \operatorname{Lie}_{\infty} \rightarrow \operatorname{Tw} \Lambda \mathrm{Lie}_{\infty}
$$

from Lemma 3.11, we obtain a morphism of dg operads

$$
\beta_{\mathcal{O}}:=\operatorname{Tw}(\hat{\varphi}) \circ \mathfrak{T}: \Lambda \operatorname{Lie}_{\infty} \rightarrow \operatorname{Tw} \mathcal{O} .
$$

From Lemma 4.1 it is then evident that the following diagram commutes:

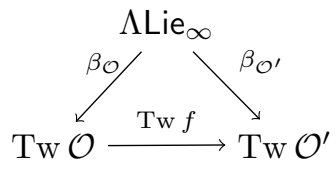

Here $f, \mathcal{O}, \mathcal{O}^{\prime}$ are as above. Summarizing, we obtain the following result:

Corollary 4.2. The operation $\mathrm{Tw}$ defines an endofunctor on the under-category $\Lambda \mathrm{Lie}_{\infty} \downarrow$ Operads.

Remark 4.3. Let $*$ be the initial object 2.3 in the category of $d g$ operads. It is easy to see that $\mathrm{Tw} *$ carries the obvious augmentation

$$
\mathrm{Tw} * \rightarrow * .
$$

Thus, if the $d g$ operad $\mathcal{O}$ in an object of $\Lambda \mathrm{Lie}_{\infty} \downarrow$ Operads has an augmentation morphism $\varepsilon: \mathcal{O} \rightarrow *$ then $\mathrm{Tw} \mathcal{O}$ is canonically augmented. The desired augmentation $\mathrm{Tw} \mathcal{O} \rightarrow *$ is obtained by composing

$$
\operatorname{Tw}(\varepsilon): \operatorname{Tw} \mathcal{O} \rightarrow \mathrm{Tw} *
$$

with 4.3).

4.1.2. The natural projection. Let $\Lambda \mathrm{Lie}_{\infty} \rightarrow \mathcal{O}$ be an arrow in Operads. There is the natural map

$$
\eta_{\mathcal{O}}: \operatorname{Tw} \mathcal{O} \rightarrow \mathcal{O}
$$

projecting the product

$$
\operatorname{Tw} \mathcal{O}(n)=\prod_{r \geq 0} \operatorname{Hom}_{S_{r}}\left(\mathbf{s}^{-2 r} \mathbb{K}, \mathcal{O}(r+n)\right)
$$

to its first $(r=0)$ factor. It is easy to see that (4.4) is a map of dg operads.

We claim that

Lemma 4.4. The maps $\eta_{\mathcal{O}}$ assemble to form a natural transformation $\eta: \mathrm{Tw} \Rightarrow i d$, where id is the identity functor on $\Lambda \mathrm{Lie}_{\infty} \downarrow$ Operads. 
Proof. We have to show that for all arrows $\Lambda$ Lie $_{\infty} \rightarrow \mathcal{O} \stackrel{f}{\longrightarrow} \mathcal{O}^{\prime}$ the following diagram commutes.

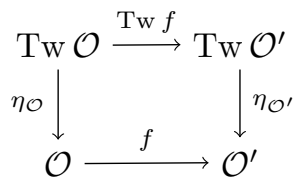

This is obvious.

4.1.3. The comultiplication $\mathfrak{D}: \mathrm{Tw} \rightarrow \mathrm{Tw} \circ \mathrm{Tw}$. Let again $\Lambda$ Lie $_{\infty} \rightarrow \mathcal{O}$ be an arrow in Operads. Consider $\operatorname{Tw} \mathrm{Tw} \mathcal{O}=\prod_{r, s \geq 0} \operatorname{Hom}_{S_{r}}\left(\mathbf{s}^{-2 r} \mathbb{K}, \operatorname{Hom}_{S_{s}}\left(\mathbf{s}^{-2 s} \mathbb{K}, \mathcal{O}(s+r+n)\right)\right) \cong \prod_{r, s \geq 0} \operatorname{Hom}_{S_{s} \times S_{r}}\left(\mathbf{s}^{-2 s-2 r} \mathbb{K}, \mathcal{O}(s+r+n)\right)$.

There are natural inclusions

$$
\operatorname{Hom}_{S_{s+r}}\left(\mathbf{s}^{-2 s-2 r} \mathbb{K}, \mathcal{O}(s+r+n)\right) \rightarrow \operatorname{Hom}_{S_{s} \times S_{r}}\left(\mathbf{s}^{-2 s-2 r} \mathbb{K}, \mathcal{O}(s+r+n)\right)
$$

and they assemble to form a map

$$
\mathfrak{D}_{\mathcal{O}}: \operatorname{Tw} \mathcal{O} \rightarrow \operatorname{Tw} \operatorname{Tw} \mathcal{O}
$$

which is alternatively defined by the equation:

$$
\mathfrak{D}_{\mathcal{O}}(f)\left(1_{r} \otimes 1_{s}\right)=f\left(1_{s+r}\right), \quad f \in \mathrm{Tw} \mathcal{O}(n) .
$$

We claim that

Lemma 4.5. The map $\mathfrak{D}_{\mathcal{O}}$ is a map of operads and the diagram

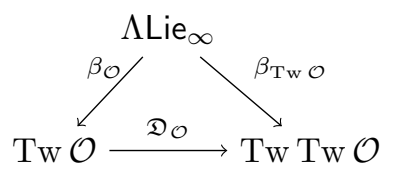

commutes.

Proof. Let us consider commutativity of diagram 4.7 first.

By definition of $\beta_{\mathcal{O}}(4.2$, we have

$$
\beta_{\mathcal{O}}\left(\mathbf{s} 1_{n}^{\mathfrak{c}}\right)\left(1_{r}\right)=\varphi_{r+n}
$$

where $\varphi_{n}:=\hat{\varphi}\left(\mathbf{s} 1_{n}^{\mathfrak{c}}\right)$.

On the other hand,

$$
\beta_{\mathrm{Tw} \mathcal{O}}=\operatorname{Tw}\left(\beta_{\mathcal{O}}\right) \circ \mathfrak{T}
$$

Hence

$$
\beta_{\mathrm{Tw} \mathcal{O}}\left(\mathbf{s} 1_{n}^{\mathfrak{c}}\right)\left(1_{r} \otimes 1_{s}\right)=\beta_{\mathcal{O}}\left(\mathbf{s} 1_{r+n}^{\mathfrak{c}}\right)\left(1_{s}\right)=\varphi_{s+r+n} .
$$

Composing $\beta_{\mathcal{O}}$ with $\mathfrak{D}_{\mathcal{O}}$, we get

$$
\mathfrak{D}_{\mathcal{O}} \circ \beta_{\mathcal{O}}\left(\mathbf{s} 1_{n}^{\mathfrak{c}}\right)\left(1_{r} \otimes 1_{s}\right)=\beta_{\mathcal{O}}\left(\mathbf{s} 1_{n}^{\mathfrak{c}}\right)\left(1_{r+s}\right)=\varphi_{s+r+n} .
$$

Comparing this result with 4.9 we conclude that diagram 4.7 indeed commutes.

Let us now show that $\mathfrak{D}_{\mathcal{O}}$ is a map of operads. 
For this purpose we consider a pair of vectors $f \in \operatorname{Tw} \mathcal{O}(n)$ and $g \in \operatorname{Tw} \mathcal{O}(m)$ and compute

$$
\mathfrak{D}_{\mathcal{O}}\left(f \circ_{i} g\right)\left(1_{r} \otimes 1_{s}\right)=f \circ_{i} g\left(1_{r+s}\right)=\sum_{p=0}^{r+s-p} \sum_{\sigma \in \operatorname{Sh}_{p, r+s-p}} \sigma \circ \varrho_{r+s, p, i}\left(f\left(1_{p}\right) \circ_{p+i} g\left(1_{r+s-p}\right)\right)
$$

where the family of permutations $\left\{\varrho_{r, p, i}\right\}_{p \leq r}$ is defined in 3.26 .

On the other hand,

$$
\begin{aligned}
\left(\mathfrak{D}_{\mathcal{O}}(f) \circ_{i} \mathfrak{D}_{\mathcal{O}}(g)\right)\left(1_{r} \otimes 1_{s}\right) & =\sum_{p_{1}=0}^{r} \sum_{\tau \in \operatorname{Sh}_{p_{1}, r-p_{1}}} \tau \circ \varrho_{r, p_{1}, i}\left(\mathfrak{D}_{\mathcal{O}}(f)\left(1_{p_{1}}\right) \circ_{p_{1}+i} \mathfrak{D}_{\mathcal{O}}(g)\left(1_{r-p_{1}}\right)\right)\left(1_{s}\right) \\
& =\sum_{\substack{0 \leq p_{1} \leq r \\
0 \leq p_{2} \leq s}} \sum_{\sigma \in T_{r, s, p_{1}, p_{2}}} \mu_{\mathbf{t}_{\sigma}}\left(\mathfrak{D}_{\mathcal{O}}(f)\left(1_{p_{1}} \otimes 1_{p_{2}}\right) \otimes \mathfrak{D}_{\mathcal{O}}(g)\left(1_{r-p_{1}} \otimes 1_{s-p_{2}}\right)\right) \\
& =\sum_{\substack{0 \leq p_{1} \leq r \\
0 \leq p_{2} \leq s}} \sum_{\sigma \in T_{r, s, p_{1}, p_{2}}} \mu_{\mathbf{t}_{\sigma}}\left(f\left(1_{p_{1}+p_{2}}\right) \otimes g\left(1_{r+s-p_{1}-p_{2}}\right)\right),
\end{aligned}
$$

where $\mathbf{t}_{\sigma}$ is the labeled planar tree depicted on figure 4.1 and the set $T_{r, s, p_{1}, p_{2}}$ consists of shuffles $\sigma \in$ $\mathrm{Sh}_{p_{1}+p_{2}, r+s-p_{1}-p_{2}}$ satisfying the conditions:

$$
\begin{gathered}
\sigma(1), \sigma(2), \ldots, \sigma\left(p_{2}\right) \in\{1,2, \ldots, s\}, \\
\sigma\left(p_{2}+p_{1}+1\right), \sigma\left(p_{2}+p_{1}+2\right), \ldots, \sigma\left(p_{1}+s\right) \in\{1,2, \ldots, s\}, \\
\sigma\left(p_{2}+1\right), \sigma\left(p_{2}+2\right), \ldots, \sigma\left(p_{2}+p_{1}\right) \in\{s+1, s+2, \ldots, s+r\}, \\
\sigma\left(p_{1}+s+1\right), \sigma\left(p_{1}+s+2\right), \ldots, \sigma(s+r) \in\{s+1, s+2, \ldots, s+r\} .
\end{gathered}
$$

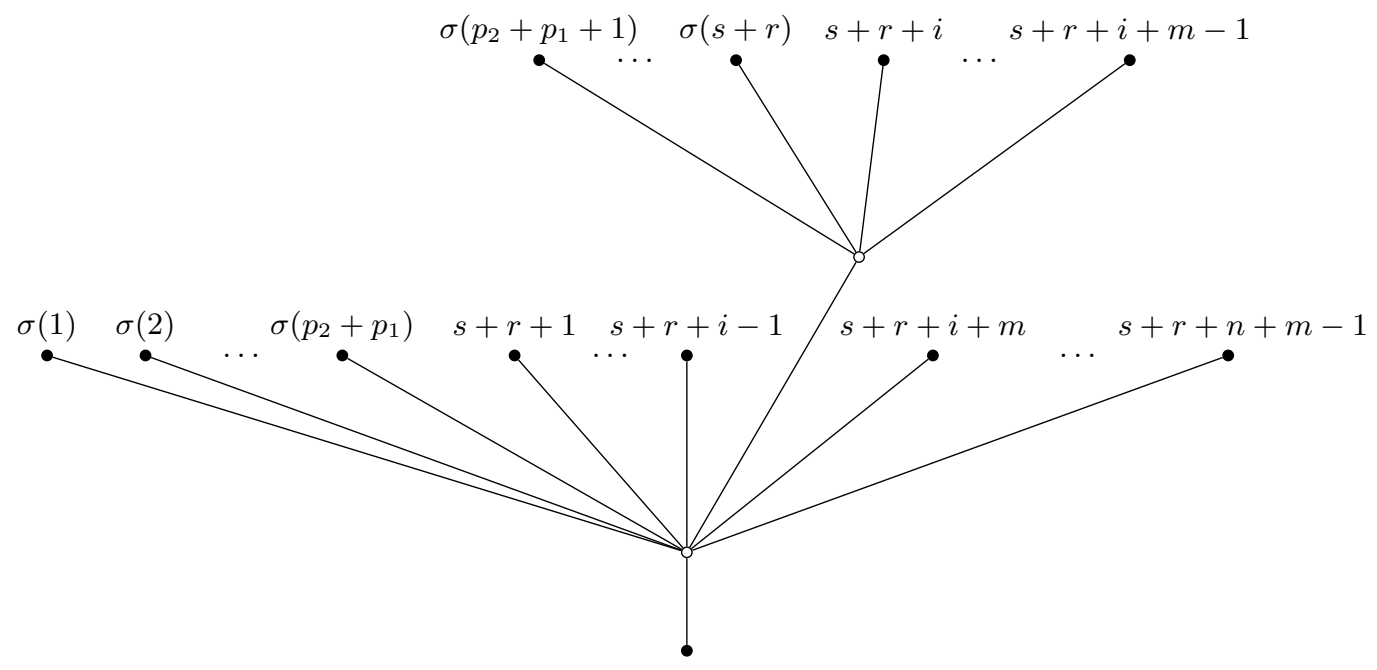

FIG. 4.1. The labeled planar tree $\mathbf{t}_{\sigma}$

It is clear that for every $0 \leq p \leq r+s$ the (disjoint) union

$$
\bigsqcup_{p_{1}+p_{2}=p} T_{r, s, p_{1}, p_{2}}
$$

coincides with the set $\mathrm{Sh}_{p, r+s-p}$. 
Hence,

$\sum_{\substack{0 \leq p_{1} \leq r \\ 0 \leq p_{2} \leq s}} \sum_{\sigma \in T_{r, s, p_{1}, p_{2}}} \mu_{\mathbf{t}_{\sigma}}\left(f\left(1_{p_{1}+p_{2}}\right) \otimes g\left(1_{r+s-p_{1}-p_{2}}\right)\right)=\sum_{p=0}^{r+s-p} \sum_{\sigma \in \operatorname{Sh}_{p, r+s-p}} \sigma \circ \varrho_{r+s, p, i}\left(f\left(1_{p}\right) \circ_{p+i} g\left(1_{r+s-p}\right)\right)$.

Thus,

$$
\mathfrak{D}_{\mathcal{O}}\left(f \circ_{i} g\right)=\mathfrak{D}_{\mathcal{O}}(f) \circ_{i} \mathfrak{D}_{\mathcal{O}}(g)
$$

In other words, $\mathfrak{D}_{\mathcal{O}}$ is compatible with all elementary operadic insertions.

It is easy to see that $\mathfrak{D}_{\mathcal{O}}$ is compatible with the units.

Lemma 4.5 is proved.

Lemma 4.6. The maps $\mathfrak{D}_{\mathcal{O}}$ 4.6 assemble to form a natural transformation $\mathfrak{D}: \mathrm{Tw} \Rightarrow \mathrm{Tw} \circ \mathrm{Tw}$.

Proof. We have to show that for all arrows $f: \mathcal{O} \rightarrow \mathcal{O}^{\prime}$ (respecting the maps from $\Lambda$ Lie $_{\infty}$ ) the following diagram commutes.

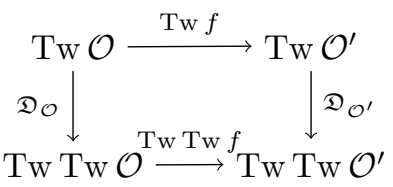

Unravelling the definitions this is amounts to saying that the following diagrams commute

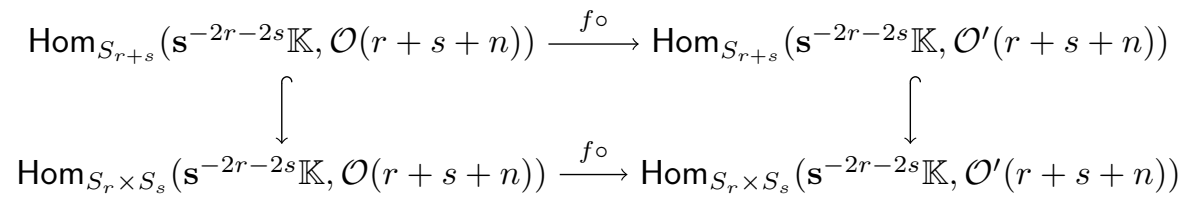

for all $r, s, n$. This is clear.

Theorem 4.7. The functor $\mathrm{Tw}$ together with the natural transformations $\eta, \mathfrak{D}$ (4.4), 4.6), is a comonad on the under-category $\Lambda \mathrm{Lie}_{\infty} \downarrow$ Operads.

Proof. We have to verify the defining relations for a comonad. The two co-unit relations boil down to the statement that for any operad $\mathcal{O}$ the compositions

$$
\begin{aligned}
& \operatorname{Tw} \mathcal{O} \stackrel{\mathfrak{D}_{\mathcal{O}}}{\longrightarrow} \operatorname{Tw} \operatorname{Tw} \mathcal{O} \stackrel{\eta_{\mathrm{Tw}} \mathcal{O}}{\longrightarrow} \operatorname{Tw} \mathcal{O} \\
& \operatorname{Tw} \mathcal{O} \stackrel{\mathfrak{D}_{\mathcal{O}}}{\longrightarrow} \operatorname{Tw} \operatorname{Tw} \mathcal{O} \stackrel{\mathrm{Tw}_{\mathfrak{O}}}{\longrightarrow} \operatorname{Tw} \mathcal{O}
\end{aligned}
$$

are the identity maps on $\mathrm{Tw} \mathcal{O}$. This statement follows immediately from the definitions. Next consider the co-associativity axiom. In our case it boils down to the statement that for any operad $\mathcal{O}$ the diagram

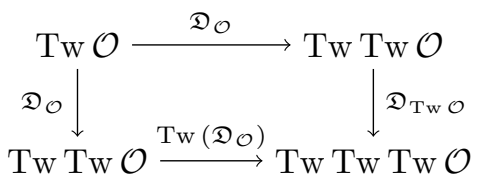


commutes. Unravelling the definitions, we have to show that the following diagram commutes

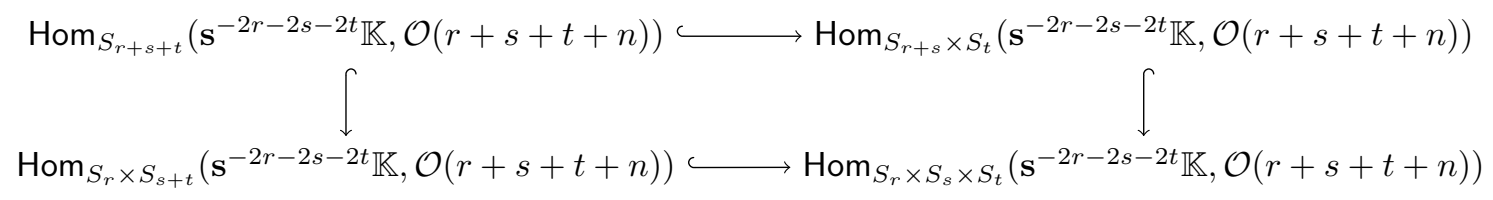

for all $r, s, t, n$. This is again clear.

4.2. Coalgebras over the comonad Tw . Let us now consider coalgebras over the comonad Tw. These are arrows of operads $\Lambda$ Lie $_{\infty} \rightarrow \mathcal{O}$ together with an operad map

$$
c: \mathcal{O} \rightarrow \operatorname{Tw} \mathcal{O}
$$

such that the following axioms hold:

- The following diagram commutes:

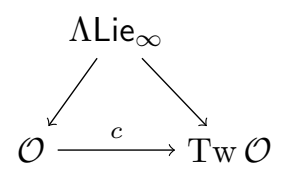

- The composition

$$
\mathcal{O} \stackrel{c}{\rightarrow} \mathrm{Tw} \mathcal{O} \stackrel{\eta_{\mathcal{O}}}{\rightarrow} \mathcal{O}
$$

is the identity.

- The following diagram commutes:

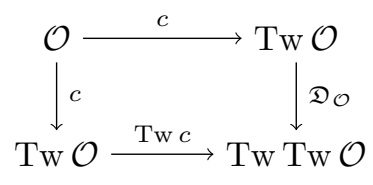

Remark 4.8. It often happens that a map from the $d g$ operad $\Lambda$ Lie $_{\infty}$ to $\mathcal{O}$ is clear from the context. In this case, we abuse the notation and say that $\mathcal{O}$ is a $\mathrm{Tw}$-coalgebra if the corresponding arrow $\Lambda$ Lie ${ }_{\infty} \rightarrow \mathcal{O}$ carries a coalgebra structure over the comonad $\mathrm{Tw}$.

Example 4.9. In Subsection 5.1.1 below, we will show that the operads $\Lambda$ Lie and Ger carry canonical structures of a Tw-coalgebra. Furthermore, it is not hard to see that, the canonical morphism of dg operads

$$
\mathfrak{T}: \Lambda \mathrm{Lie}_{\infty} \rightarrow \mathrm{Tw} \Lambda \mathrm{Lie}_{\infty}
$$

from Lemma 3.11 equips $\Lambda \mathrm{Lie}_{\infty}$ with a structure of a $\mathrm{Tw}$-coalgebra.

Example 4.10. If $\Lambda \mathrm{Lie}_{\infty} \rightarrow \mathcal{O}$ is an arrow, then $\mathrm{Tw} \mathcal{O}$ is a $\mathrm{Tw}$-coalgebra (a cofree $\mathrm{Tw}$-coalgebra).

Another example of a $\mathrm{Tw}$-coalgebra is given in the next section. 
4.3. $\mathrm{Ger}_{\infty}$ is canonically a Tw-coalgebra. Let us recall from [14, [15, [18, that

$$
\operatorname{Ger}_{\infty}:=\operatorname{Cobar}\left(\operatorname{Ger}^{\vee}\right)
$$

where $\mathrm{Ger}^{\vee}$ is the cooperad Koszul dual to Ger. Concretely, Ger ${ }^{\vee}$ is obtained from $\Lambda^{-2} \mathrm{Ger}$ by taking the linear dual. The operad $\Lambda \mathrm{Lie}_{\infty}$ is a sub-operad of $\mathrm{Ger}_{\infty}$, and we denote the inclusion by

$$
\iota: \Lambda \mathrm{Lie}_{\infty} \rightarrow \mathrm{Ger}_{\infty} .
$$

Our goal, in this section, is to show that $\mathrm{Ger}_{\infty}$ is a $\mathrm{Tw}$-coalgebra. In order to do this, we have to complete two tasks:

(1) Construct a map of $\operatorname{dg}$ operads $c: \mathrm{Ger}_{\infty} \rightarrow \mathrm{Tw}_{\mathrm{Wer}}$.

(2) Verify that $c$ satisfies the axioms for a Tw-coalgebra.

To complete the first task we consider the free $\Lambda^{-2}$ Ger-algebra $\Lambda^{-2} \mathrm{Ger}_{n}$ in $n$ dummy variables $b_{1}, \ldots, b_{n}$ of degree zero. The $n$-th space $\Lambda^{-2} \operatorname{Ger}(n)$ of the operad $\Lambda^{-2}$ Ger is spanned by $\Lambda^{-2}$ Ger-monomials in $b_{1}, \ldots, b_{n}$ in which each dummy variable $b_{i}$ appears exactly once. It is clear that the operad $\Lambda^{-2}$ Ger is generated by the two vectors $b_{1} b_{2},\left\{b_{1}, b_{2}\right\} \in \Lambda^{-2} \operatorname{Ger}(2)$ of degrees 2 and 1 , respectively.

Next, we consider the ordered partitions of the set $\{1,2, \ldots, n\}$

$$
\left\{i_{11}, i_{12}, \ldots, i_{1 p_{1}}\right\} \sqcup\left\{i_{21}, i_{22}, \ldots, i_{2 p_{2}}\right\} \sqcup \cdots \sqcup\left\{i_{t 1}, i_{t 2}, \ldots, i_{t p_{t}}\right\}
$$

satisfying the following properties:

- for each $1 \leq \beta \leq t$ the index $i_{\beta p_{\beta}}$ is the biggest among $i_{\beta 1}, \ldots, i_{\beta p_{\beta}}$

- $i_{1 p_{1}}<i_{2 p_{2}}<\cdots<i_{t p_{t}}$ (in particular, $i_{t p_{t}}=n$ ).

It is not hard to see that for each $n \geq 1$ the monomials

$$
\left\{b_{i_{11}}, \ldots,\left\{b_{i_{1\left(p_{1}-1\right)}}, b_{i_{1_{1}}}\right\} .\right\} \ldots\left\{b_{i_{t 1}}, \ldots,\left\{b_{i_{t\left(p_{t}-1\right)}}, b_{i_{t p_{t}}}\right\} .\right\}
$$

corresponding to all ordered partitions 4.15 satisfying the above properties form a basis 9 of $\Lambda^{-2} \operatorname{Ger}(n)$. We denote by $I_{n}$ the set of the ordered partitions 4.15 and reserve the notation

$$
\left\{w_{n, i}\right\}_{i \in I_{n}}
$$

and

$$
\left\{w_{n, i}^{*}\right\}_{i \in I_{n}}
$$

for the basis of $\Lambda^{-2} \operatorname{Ger}(n)$ formed by monomials 4.16 and the dual basis of $\operatorname{Ger}^{\vee}(n)=\left(\Lambda^{-2} \operatorname{Ger}(n)\right)^{*}$, respectively.

We observe that, for every $r \geq 0$ and for every basis vector $w_{n, i}$, the monomial

$$
b_{1} \ldots b_{r} w_{n, i}\left(b_{r+1}, \ldots, b_{r+n}\right)
$$

belongs to the basis of $\Lambda^{-2} \operatorname{Ger}(r+n)$. In particular, we denote by

$$
\left(b_{1} \ldots b_{r} w_{n, i}\left(b_{r+1}, \ldots, b_{r+n}\right)\right)^{*}
$$

the basis vector of $\operatorname{Ger}^{\vee}(r+n)$ which is dual to $b_{1} \ldots b_{r} w_{n, i}\left(b_{r+1}, \ldots, b_{r+n}\right)$ in $\Lambda^{-2} \operatorname{Ger}(r+n)$.

\footnotetext{
${ }^{9}$ Using this fact, it is easy to see that $\operatorname{dim} \Lambda^{-2} \operatorname{Ger}(n)=n$ !
} 
Since for every $r \geq 0$ and $n \geq 2$ the element $\mathbf{s}^{2 r+1}\left(b_{1} \ldots b_{r} w_{n, i}\left(b_{r+1}, \ldots, b_{r+n}\right)\right)^{*}$ can be viewed as a vector in

$$
\mathbf{s}^{2 r+1}\left(\operatorname{Ger}^{\vee}(r+n)\right)^{S_{r}} \subset \mathrm{Tw}_{\mathrm{w}} \operatorname{Ger}_{\infty}(n),
$$

the formula

$$
\alpha_{\mathfrak{G}}=\sum_{n \geq 2, r \geq 0} \sum_{i \in I_{n}} \mathbf{s}^{2 r+1}\left(b_{1} \ldots b_{r} w_{n, i}\left(b_{r+1}, \ldots, b_{r+n}\right)\right)^{*} \otimes w_{n, i}
$$

defines a degree 1 element in the dg Lie algebra

$$
\operatorname{Conv}\left(\operatorname{Ger}_{\circ}^{\vee}, \operatorname{Tw}_{\mathrm{w}} \operatorname{Ger}_{\infty}\right) \cong \prod_{n \geq 2}\left(\operatorname{Tw}_{\mathrm{wer}}(n) \otimes \Lambda^{-2} \operatorname{Ger}(n)\right)^{S_{n}}
$$

We claim that

Proposition 4.11. Equation 4.19) defines a Maurer-Cartan element of the dg Lie algebra 4.20.

The proof of this proposition is quite technical so we postpone it to Subsection 4.3.1 given below.

Due to Theorem 2.2. maps from $\mathrm{Ger}_{\infty}$ to $\mathrm{Tw}_{\mathrm{Ger}}$ are in bijection with Maurer-Cartan elements of the dg Lie algebra 4.20). Hence the Maurer-Cartan element $\alpha_{\mathfrak{G}} 4.19$ ) defines a map of dg operads

$$
c: \mathrm{Ger}_{\infty} \rightarrow \mathrm{Tw} \mathrm{Ger}_{\infty} .
$$

To prove that $c$ equips $\mathrm{Ger}_{\infty}$ with the structure of a $\mathrm{Tw}$-coalgebra, we have to verify three conditions. The first condition states that the following diagram shall commute:

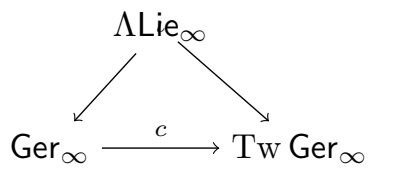

Here the right hand arrow is defined as the composition

$$
\Lambda \mathrm{Lie}_{\infty} \rightarrow \mathrm{Tw} \Lambda \mathrm{Lie}_{\infty} \stackrel{\mathrm{Tw} \iota}{\longrightarrow} \mathrm{Tw}_{\mathrm{Ger}} .
$$

It sends the generator $\mathbf{s} 1_{n}^{\mathfrak{c}}$ of $\Lambda \mathrm{Lie}_{\infty}$ to (cf. 3.45)

$$
\sum_{r \geq 0} \mathbf{s}^{2 r+1}\left(b_{1} b_{2} \cdots b_{r+n}\right)^{*} .
$$

On the other hand, the left and bottom morphisms in the diagram under consideration send $\mathbf{s} 1_{n}^{\mathfrak{c}}$ to

$$
c \circ \iota\left(\mathbf{s} 1_{n}^{\mathfrak{c}}\right)=c\left(\mathbf{s}\left(b_{1} \cdots b_{n}\right)^{*}\right)=\sum_{r \geq 0} \mathbf{s}^{2 r+1}\left(b_{1} b_{2} \cdots b_{r+n}\right)^{*} .
$$

Hence this condition is satisfied. The second condition for Tw-coalgebras to be checked says that the composition

$$
\mathrm{Ger}_{\infty} \rightarrow \mathrm{Tw} \mathrm{Ger}_{\infty} \rightarrow \mathrm{Ger}_{\infty}
$$

shall be the identity. It is obviously satisfied. The third condition states that the two compositions below shall be the same.

$$
\begin{aligned}
& \operatorname{Ger}_{\infty} \stackrel{c}{\longrightarrow} \operatorname{Tw}_{w} \operatorname{Ger}_{\infty} \stackrel{\mathfrak{D G e r}_{\infty}}{\longrightarrow} \text { Tw Tw Ger } \\
& \operatorname{Ger}_{\infty} \stackrel{c}{\longrightarrow} \mathrm{Tw}_{\infty} \operatorname{Ger}_{\infty} \stackrel{\mathrm{Tw}(c)}{\longrightarrow} \mathrm{Tw}_{\mathrm{Tw}} \mathrm{Ger}_{\infty}
\end{aligned}
$$


In fact, unfolding the definitions one finds that both compositions operate on generators $\mathbf{s} w_{n, i}^{*}$ of $\operatorname{Ger}_{\infty}$ as follows:

$$
\mathbf{s} w_{n, i}^{*} \mapsto \sum_{r \geq 0} \sum_{s \geq 0} \mathbf{s}^{2 r+2 s+1} b_{1} \ldots b_{r+s} w_{n, i}^{*}\left(b_{r+s+1}, \ldots, b_{r+s+n}\right)
$$

Thus the third condition is also satisfied.

Summarizing, we obtain the following theorem:

Theorem 4.12. The morphism of $d g$ operads

$$
c: \mathrm{Ger}_{\infty} \rightarrow \mathrm{Tw}_{\mathrm{Ger}}^{\infty}
$$

corresponding to the Maurer-Cartan element $\alpha_{\mathfrak{G}} 4.19$ equips $\mathrm{Ger}_{\infty}$ with a Tw-coalgebra structure.

4.3.1. The proof of Proposition 4.11. The proof of Theorem4.12 is based on Proposition4.11. Here we prove this proposition.

Let $n, r$ be a pair of integers with $r \geq 0$ and $n \geq 2$. In the groupoid $\operatorname{Tree}_{2}(r+n)$ we consider the objects $\mathbf{t}_{\sigma}^{\text {below }}, \mathbf{t}_{\sigma}^{\text {above }}, \mathbf{t}_{\sigma}^{k}, \mathbf{t}_{\sigma, \tau}^{p, q}$ shown on figures $4.2,4.34 .4$, and 4.5 , respectively.

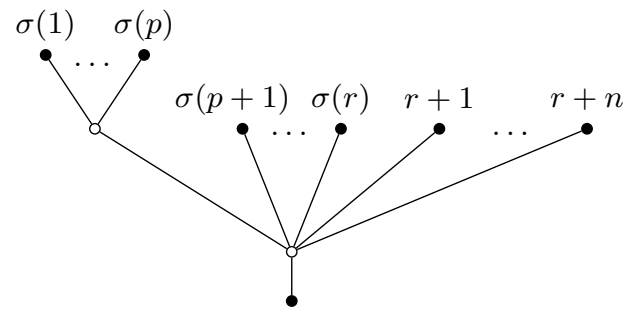

Fig. 4.2. The tree $\mathbf{t}_{\sigma}^{\text {below }}$. Here $2 \leq p \leq r$ and $\sigma \in \mathrm{Sh}_{p, r-p}$

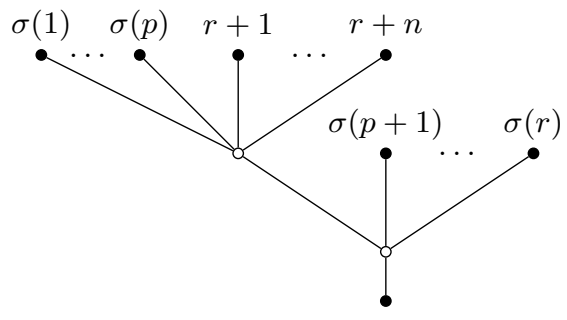

FIG. 4.3. The tree $\mathbf{t}_{\sigma}^{\text {above }}$. Here $0 \leq p \leq r-1$ and $\sigma \in \mathrm{Sh}_{p, r-p}$

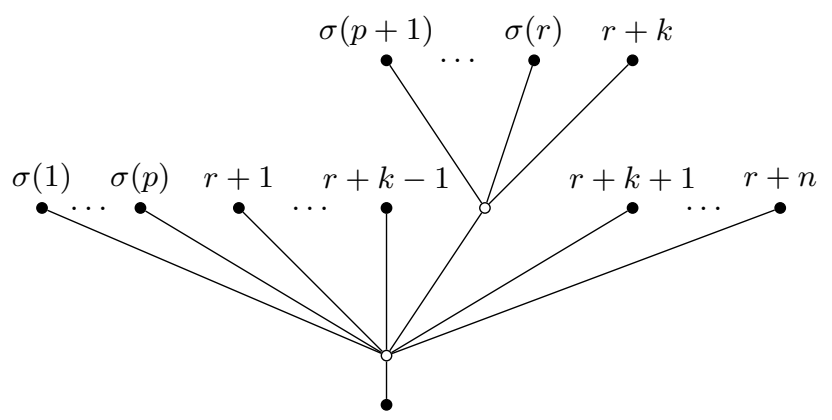

FIG. 4.4. The tree $\mathbf{t}_{\sigma}^{k}$. Here $2-n \leq p \leq r-1,1 \leq k \leq n$, and $\sigma \in \mathrm{Sh}_{p, r-p}$

It is clear that the trees $\mathbf{t}_{\sigma}^{\text {below }}, \mathbf{t}_{\sigma}^{\text {above }}, \mathbf{t}_{\sigma}^{k}, \mathbf{t}_{\sigma, \tau}^{p, q}$ are all mutually non-isomorphic. Furthermore, if both nodal vertices of a tree $\mathbf{t} \in \operatorname{Tree}_{2}(n)$ have valencies $\geq 3$, then $\mathbf{t}$ is isomorphic to one of the trees in the list: $\mathbf{t}_{\sigma}^{\text {below }}, \mathbf{t}_{\sigma}^{\text {above }}, \mathbf{t}_{\sigma}^{k}, \mathbf{t}_{\sigma, \tau}^{p, q}$.

We will need the following technical statement:

Lemma 4.13. Let $n, r$ be a pair of integers with $r \geq 0, n \geq 2, w_{n, i}$ be a vector in the basis (4.17), and

$$
\left(b_{1} \ldots b_{r} w_{n, i}\left(b_{r+1}, \ldots, b_{r+n}\right)\right)^{*}
$$




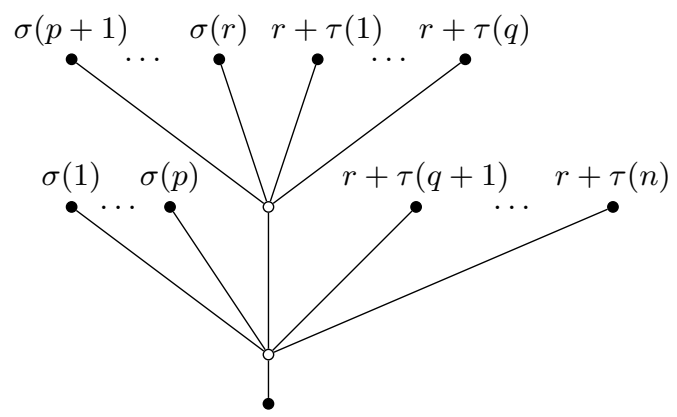

FIG. 4.5. The tree $\mathbf{t}_{\sigma, \tau}^{p, q}$. Here $0 \leq p \leq r, 2 \leq q \leq n-1, \sigma \in \mathrm{Sh}_{p, r-p}$ and $\tau \in \mathrm{Sh}_{q, n-q}$

be the basis vector of $\operatorname{Ger}^{\vee}(r+n)$ dual to $b_{1} \ldots b_{r} w_{n, i}\left(b_{r+1}, \ldots, b_{r+n}\right) \in \Lambda^{-2} \operatorname{Ger}(r+n)$. Then

$$
\begin{gathered}
\partial^{\text {Cobar }} \mathbf{s}\left(b_{1} \ldots b_{r} w_{n, i}\left(b_{r+1}, \ldots, b_{r+n}\right)\right)^{*}= \\
-\sum_{\substack{2 \leq p \leq r \\
\sigma \in \operatorname{Sh}_{p, r-p}}}(-1)^{\left|w_{n, i}\right|}\left(\mathbf{t}_{\sigma}^{\text {below }} ; \mathbf{s}\left(b_{1} \ldots b_{r-p+1} w_{n, i}\left(b_{r-p+2}, \ldots, b_{r-p+1+n}\right)\right)^{*} \otimes \mathbf{s}\left(b_{1} \ldots b_{p}\right)^{*}\right) \\
-\sum_{\substack{0 \leq p \leq r-1 \\
\sigma \in \operatorname{Sh}_{p, r-p}}}\left(\mathbf{t}_{\sigma}^{\text {above }} ; \mathbf{s}\left(b_{1} \ldots b_{r-p+1}\right)^{*} \otimes \mathbf{s}\left(b_{1} \ldots b_{p} w_{n, i}\left(b_{p+1}, \ldots, b_{p+n}\right)\right)^{*}\right) \\
-\sum_{\substack{2-n \leq p \leq r-1 \\
\sigma \in \operatorname{Sh}_{p, r-p}}} \sum_{\substack{k=1 \\
0 \leq p \leq r}}^{n}(-1)^{\left|w_{n, i}\right|}\left(\mathbf{t}_{\sigma}^{k} ; \mathbf{s}\left(b_{1} \ldots b_{p} w_{n, i}\left(b_{p+1}, \ldots, b_{p+n}\right)\right)^{*} \otimes \mathbf{s}\left(b_{1} \ldots b_{r-p+1}\right)^{*}\right) \\
\sum_{\substack{\sigma \leq \mathrm{Sh}_{p, r-p} \\
\tau \in \mathrm{Sh}_{q, n-q}}} \sum_{i_{1, i}, i_{2}}(-1)^{\left|w_{n-q+1, i_{1}}\right|\left|w_{q, i_{2}}\right|+\left|w_{n-q+1, i_{1}}\right|} f_{i_{1} i_{2}}^{i}\left(\mathbf{t}_{\sigma, \tau}^{p, q} ; \mathbf{s}\left(b_{1} \ldots b_{p} w_{n-q+1, i_{1}}\left(b_{p+1}, \ldots, b_{p+n-q+1}\right)\right)^{*}\right.
\end{gathered}
$$

$$
\left.\otimes \mathbf{s}\left(b_{1} \ldots b_{r-p} w_{q, i_{2}}\left(b_{r-p+1}, \ldots, b_{r-p+q}\right)\right)^{*}\right),
$$

where the coefficients $f_{i_{1} i_{2}}^{i} \in \mathbb{K}$ are defined by the equation

$$
\tau\left(w_{n-q+1, i_{1}} \circ_{1} w_{q, i_{2}}\right)=\sum_{i \in I_{n}} f_{i_{1} i_{2}}^{i} w_{n, i} .
$$

Proof. The statement of the lemma follows from these equations:

$$
\begin{gathered}
\Delta_{\mathbf{t}_{\sigma}^{\text {below }}}\left(b_{1} \ldots b_{r} w_{n, i}\left(b_{r+1}, \ldots, b_{r+n}\right)\right)^{*}= \\
\left(b_{1} \ldots b_{r-p+1} w_{n, i}\left(b_{r-p+2}, \ldots, b_{r-p+1+n}\right)\right)^{*} \otimes\left(b_{1} \ldots b_{p}\right)^{*}, \\
\Delta_{\mathbf{t}_{\sigma}^{\text {above }}}\left(b_{1} \ldots b_{r} w_{n, i}\left(b_{r+1}, \ldots, b_{r+n}\right)\right)^{*}= \\
\left(b_{1} \ldots b_{r-p+1}\right)^{*} \otimes\left(b_{1} \ldots b_{p} w_{n, i}\left(b_{p+1}, \ldots, b_{p+n}\right)\right)^{*}, \\
\Delta_{\mathbf{t}_{\sigma}^{k}}\left(b_{1} \ldots b_{r} w_{n, i}\left(b_{r+1}, \ldots, b_{r+n}\right)\right)^{*}= \\
\left(b_{1} \ldots b_{p} w_{n, i}\left(b_{p+1}, \ldots, b_{p+n}\right)\right)^{*} \otimes\left(b_{1} \ldots b_{r-p+1}\right)^{*},
\end{gathered}
$$

and

$$
\Delta_{\mathbf{t}_{\sigma, \tau}^{p, q}}\left(b_{1} \ldots b_{r} w_{n, i}\left(b_{r+1}, \ldots, b_{r+n}\right)\right)^{*}=
$$




$$
\begin{gathered}
\sum_{\substack{i_{1} \in I_{n-q+1} \\
i_{2} \in I_{q}}}(-1)^{\left|w_{n-q+1, i_{1}}\right| \cdot\left|w_{q, i_{2}}\right|} f_{i_{1} i_{2}}^{i}\left(b_{1} \ldots b_{p} w_{n-q+1, i_{1}}\left(b_{p+1}, \ldots, b_{p+n-q+1}\right)\right)^{*} \\
\otimes\left(b_{1} \ldots b_{r-p} w_{q, i_{2}}\left(b_{r-p+1}, \ldots, b_{r-p+q}\right)\right)^{*}
\end{gathered}
$$

where the coefficients $f_{i_{1} i_{2}}^{i}$ are defined by equation 4.24.

Let us prove that equation 4.28 holds.

In general, we have

$$
\begin{gathered}
\Delta_{\mathbf{t}_{\sigma, \tau}^{p, q}}\left(b_{1} \ldots b_{r} w_{n, i}\left(b_{r+1}, \ldots, b_{r+n}\right)\right)^{*}= \\
\sum_{\substack{j_{1} \in I_{n+p-q+1}, j_{2} \in I_{r-p+q}}} g_{j_{1}, j_{2}}^{i} w_{n+p-q+1, j_{1}}^{*} \otimes w_{r-p+q, j_{2}}^{*}, \quad g_{j_{1} j_{2}}^{i} \in \mathbb{K} .
\end{gathered}
$$

It is not hard to see that, if the basis vector $w_{n+p-q+1, j_{1}}$ is not of the form

$$
b_{1} \ldots b_{p} w_{n-q+1, i_{1}}\left(b_{p+1}, \ldots, b_{p+n-q+1}\right)
$$

or the basis vector $w_{r-p+q, j_{2}}$ is not of the form

$$
b_{1} \ldots b_{r-p} w_{q, i_{2}}\left(b_{r-p+1}, \ldots, b_{r-p+q}\right) \text {, }
$$

then

$$
\left(b_{1} \ldots b_{r} w_{n, i}\left(b_{r+1}, \ldots, b_{r+n}\right)\right)^{*}\left(\mu_{\mathbf{t}_{\sigma, \tau}^{p, q}}\left(w_{n+p-q+1, j_{1}} \otimes w_{r-p+q, j_{2}}\right)=0 .\right.
$$

In other words, $g_{j_{1} j_{2}}^{i}=0$ unless the basis vector $w_{n+p-q+1, j_{1}}$ is of the form

$$
b_{1} \ldots b_{p} w_{n-q+1, i_{1}}\left(b_{p+1}, \ldots, b_{p+n-q+1}\right)
$$

and the basis vector $w_{r-p+q, j_{2}}$ is of the form

$$
b_{1} \ldots b_{r-p} w_{q, i_{2}}\left(b_{r-p+1}, \ldots, b_{r-p+q}\right) .
$$

Hence,

$$
\begin{gathered}
\Delta_{\mathbf{t}_{\sigma, \tau}^{p, q}}\left(b_{1} \ldots b_{r} w_{n, i}\left(b_{r+1}, \ldots, b_{r+n}\right)\right)^{*}= \\
\sum_{\substack{i_{1} \in I_{n-q+1} \\
i_{2} \in I_{q}}} \widetilde{f}_{i_{1} i_{2}}^{i}\left(b_{1} \ldots b_{p} w_{n-q+1, i_{1}}\left(b_{p+1}, \ldots, b_{p+n-q+1}\right)\right)^{*} \otimes\left(b_{1} \ldots b_{r-p} w_{q, i_{2}}\left(b_{r-p+1}, \ldots, b_{r-p+q}\right)\right)^{*}
\end{gathered}
$$

for some coefficients $\widetilde{f}_{i_{1} i_{2}}^{i} \in \mathbb{K}$.

On the other hand, we have

$$
\begin{gathered}
\Delta_{\mathbf{t}_{\sigma, \tau}^{p, q}}\left(b_{1} \ldots b_{r} w_{n, i}\left(b_{r+1}, \ldots, b_{r+n}\right)\right)^{*}\left(b_{1} \ldots b_{p} w_{n-q+1, i_{1}}\left(b_{p+1}, \ldots, b_{p+n-q+1}\right)\right. \\
\left.\otimes b_{1} \ldots b_{r-p} w_{q, i_{2}}\left(b_{r-p+1}, \ldots, b_{r-p+q}\right)\right)= \\
\left(b_{1} \ldots b_{r} w_{n, i}\left(b_{r+1}, \ldots, b_{r+n}\right)\right)^{*}\left(\mu _ { \mathbf { t } _ { \sigma , \tau } ^ { p , q } } \left(b_{1} \ldots b_{p} w_{n-q+1, i_{1}}\left(b_{p+1}, \ldots, b_{p+n-q+1}\right)\right.\right. \\
\left.\left.\otimes b_{1} \ldots b_{r-p} w_{q, i_{2}}\left(b_{r-p+1}, \ldots, b_{r-p+q}\right)\right)\right)= \\
(-1)^{\varepsilon_{i_{1} i_{2}}}\left(b_{1} \ldots b_{r} w_{n, i}\left(b_{r+1}, \ldots, b_{r+n}\right)\right)^{*}\left(b_{\sigma(1)} \ldots b_{\sigma(p)}\right. \\
\left.w_{n-q+1, i_{1}}\left(b_{\sigma(p+1)} \ldots b_{\sigma(r)} w_{q, i_{2}}\left(b_{r+\tau(1)}, \ldots, b_{r+\tau(q)}\right), b_{r+\tau(q+1)}, \ldots, b_{r+\tau(n)}\right)\right),
\end{gathered}
$$

where the sign factor $(-1)^{\varepsilon_{i_{1} i_{2}}}$ comes from permuting the $\Lambda^{-2}$ Ger-monomial $w_{q, i_{2}}$ with brackets $\{$,$\} .$ 
Since in the variables $b_{1}, \ldots, b_{r}$ in the $\Lambda^{-2}$ Ger-monomial $b_{1} \ldots b_{r} w_{n, i}\left(b_{r+1}, \ldots, b_{r+n}\right)$ only enter $\Lambda^{-1}$ Lie words of length 1 , we have

$$
\begin{gathered}
\Delta_{\mathbf{t}_{\sigma, \tau}^{p, q}}\left(b_{1} \ldots b_{r} w_{n, i}\left(b_{r+1}, \ldots, b_{r+n}\right)\right)^{*}\left(b_{1} \ldots b_{p} w_{n-q+1, i_{1}}\left(b_{p+1}, \ldots, b_{p+n-q+1}\right)\right. \\
\left.\otimes b_{1} \ldots b_{r-p} w_{q, i_{2}}\left(b_{r-p+1}, \ldots, b_{r-p+q}\right)\right)= \\
(-1)^{\varepsilon_{i_{1} i_{2}}}\left(b_{1} \ldots b_{r} w_{n, i}\left(b_{r+1}, \ldots, b_{r+n}\right)\right)^{*}\left(b_{\sigma(1)} \ldots b_{\sigma(r)}\right. \\
\left.w_{n-q+1, i_{1}}\left(w_{q, i_{2}}\left(b_{r+\tau(1)}, \ldots, b_{r+\tau(q)}\right), b_{r+\tau(q+1)}, \ldots, b_{r+\tau(n)}\right)\right)= \\
(-1)^{\varepsilon_{i_{1} i_{2}}}\left(b_{1} \ldots b_{r} w_{n, i}\left(b_{r+1}, \ldots, b_{r+n}\right)\right)^{*}\left(b_{1} \ldots b_{r}\right. \\
\left.w_{n-q+1, i_{1}}\left(w_{q, i_{2}}\left(b_{r+\tau(1)}, \ldots, b_{r+\tau(q)}\right), b_{r+\tau(q+1)}, \ldots, b_{r+\tau(n)}\right)\right)=f_{i_{1} i_{2}}^{i}
\end{gathered}
$$

Thus $\widetilde{f}_{i_{1} i_{2}}^{i}=(-1)^{\left|w_{n-q+1, i_{1}}\right|\left|w_{q, i_{2}}\right|} f_{i_{1} i_{2}}^{i}$ and equation 4.28 holds.

Using the identification $\mathrm{Ger}^{\vee}=\Lambda^{-2} \mathrm{Ger}^{*}$ in the similar way, it is easy to prove that equations 4.25, 4.26, 4.27) also hold.

Lemma 4.13 follows.

To prove Proposition 4.11 we need to show that the element $\alpha_{\mathfrak{G}}$ (4.19) satisfies the Maurer-Cartan equation

$$
\partial^{\mathrm{Cobar}} \alpha_{\mathfrak{G}}+\partial^{\mathrm{Tw}} \alpha_{\mathfrak{G}}+\alpha_{\mathfrak{G}} \bullet \alpha_{\mathfrak{G}}=0
$$

in the dg Lie algebra 4.20 .

Equation 4.33 unfolds as follows:

$$
\begin{gathered}
\sum_{n \geq 2, r \geq 0} \sum_{i \in I_{n}} \mathbf{s}^{2 r} \partial^{\operatorname{Cobar}}\left(\mathbf{s}\left(b_{1} \ldots b_{r} w_{n, i}\left(b_{r+1}, \ldots, b_{r+n}\right)\right)^{*}\right) \otimes w_{n, i}+ \\
\sum_{n \geq 2, r \geq 0} \sum_{i \in I_{n}} \partial^{\mathrm{Tw}}\left(\mathbf{s}^{2 r+1}\left(b_{1} \ldots b_{r} w_{n, i}\left(b_{r+1}, \ldots, b_{r+n}\right)\right)^{*}\right) \otimes w_{n, i} \\
+\sum_{\substack{0 \leq p \leq r \\
2 \leq q \leq n-1}} \sum_{\substack{\sigma \in \mathrm{Sh}_{p, r-p} \\
\tau \in \mathrm{Sh}_{q, n-q}}} \sum_{i_{1}, i_{2}}(-1)^{\left|w_{n-q+1, i_{1}}\right|\left(\left|w_{q, i_{2}}\right|+1\right)}\left(\mathbf{t}_{\sigma, \tau}^{p, q} ; \mathbf{s}^{2 p+1}\left(b_{1} \ldots b_{p} w_{n-q+1, i_{1}}\left(b_{p+1}, \ldots, b_{p+n-q+1}\right)\right)^{*}\right. \\
\left.\otimes \mathbf{s}^{2(r-p)+1}\left(b_{1} \ldots b_{r-p} w_{q, i_{2}}\left(b_{r-p+1}, \ldots, b_{r-p+q}\right)\right)^{*}\right) \otimes f_{i_{1} i_{2}}^{i} w_{n, i}=0,
\end{gathered}
$$

where the coefficients $f_{i_{1} i_{2}}^{i}$ are defined by equations 4.24 .

Let us now use Lemma 4.13 .

The contribution to

$$
\sum_{n \geq 2, r \geq 0} \sum_{i \in I_{n}} \mathbf{s}^{2 r} \partial^{\text {Cobar }}\left(\mathbf{s}\left(b_{1} \ldots b_{r} w_{n, i}\left(b_{r+1}, \ldots, b_{r+n}\right)\right)^{*}\right) \otimes w_{n, i}
$$

coming from the last sum in the right hand side of 4.23 cancels with the third sum in equation 4.34).

The contributions to

$$
\sum_{n \geq 2, r \geq 0} \sum_{i \in I_{n}} \mathbf{s}^{2 r} \partial^{\text {Cobar }}\left(\mathbf{s}\left(b_{1} \ldots b_{r} w_{n, i}\left(b_{r+1}, \ldots, b_{r+n}\right)\right)^{*}\right) \otimes w_{n, i}
$$

coming from the remaining sums in the right hand side of 4.23 cancel the sum

$$
\sum_{n \geq 2, r \geq 0} \sum_{i \in I_{n}} \partial^{\mathrm{Tw}}\left(\mathbf{s}^{2 r}\left(b_{1} \ldots b_{r} w_{n, i}\left(b_{r+1}, \ldots, b_{r+n}\right)\right)^{*}\right) \otimes w_{n, i}
$$

Thus $\alpha_{\mathfrak{G}}$ satisfies 4.33 and Proposition 4.11 follows. 


\section{Homotopy theORETiC PRoperties of the TWISTING PROCEDURE}

Let us prove that the functor $\mathrm{Tw}$ preserves quasi-isomorphisms.

Theorem 5.1. Let $\Lambda \mathrm{Lie}_{\infty} \rightarrow \mathcal{O} \stackrel{F}{\rightarrow} \mathcal{O}^{\prime}$ be a morphism of the under-category $\Lambda \mathrm{Lie}_{\infty} \downarrow$ Operads with $F$ being a quasi-isomorphism. Then, the map $\mathrm{Tw}(F): \operatorname{Tw} \mathcal{O} \rightarrow \mathrm{Tw} \mathcal{O}^{\prime}$ is also a quasi-isomorphism.

Proof. We want to show that $\operatorname{Tw} \mathcal{O}(n) \rightarrow \operatorname{Tw} \mathcal{O}^{\prime}(n)$ is a quasi-isomorphism for every $n=0,1,2, \ldots$ This is equivalent to saying that the mapping cone

$$
C:=\operatorname{Tw} \mathcal{O}(n) \oplus \mathbf{s} \operatorname{Tw} \mathcal{O}^{\prime}(n)
$$

is acyclic for every $n$. There is a natural complete filtration $C=\mathcal{F}_{0} \supset \mathcal{F}_{1} \supset \cdots$ such that

$$
\mathcal{F}_{p}=\prod_{r \geq p} \operatorname{Hom}_{S_{r}}\left(\mathbf{s}^{-2 r} \mathbb{K}, \mathcal{O}(r+n)\right) \oplus \prod_{r \geq p} \mathbf{s ~ H o m}_{S_{r}}\left(\mathbf{s}^{-2 r} \mathbb{K}, \mathcal{O}^{\prime}(r+n)\right) .
$$

Note that the associated graded complex for this filtration is

$$
\bigoplus_{p} \mathcal{F}_{p} / \mathcal{F}_{p+1} \cong \bigoplus_{p} \operatorname{Hom}_{S_{p}}\left(\mathbf{s}^{-2 p} \mathbb{K}, \mathcal{O}(p+n) \oplus \mathbf{s} \mathcal{O}^{\prime}(p+n)\right)
$$

The complex $\mathcal{O}(p+n) \oplus \mathbf{s} \mathcal{O}^{\prime}(p+n)$ is the mapping cone of $\mathcal{O}(p+n) \rightarrow \mathcal{O}^{\prime}(p+n)$ and hence acyclic by assumption. Since taking invariants with respect to a finite group action commutes with taking cohomology, we conclude that the associated graded is acyclic as well. Thus, by Lemma D.1 from Appendix D, the statement of the proposition follows.

Example 5.2. Since the canonical maps

$$
U_{\Lambda \text { Lie }}: \Lambda \text { Lie }_{\infty} \rightarrow \Lambda \text { Lie }, \quad U_{\text {Ger }}: \text { Ger }_{\infty} \rightarrow \text { Ger }
$$

are quasi-isomorphisms of dg operads, the morphisms

$$
\operatorname{Tw}\left(U_{\Lambda \text { Lie }}\right): \operatorname{Tw}\left(\Lambda \operatorname{Lie}_{\infty}\right) \rightarrow \operatorname{Tw}(\Lambda \text { Lie }),
$$

and

$$
\operatorname{Tw}\left(U_{\mathrm{Ger}}\right): \mathrm{Tw} \mathrm{Ger}_{\infty} \rightarrow \mathrm{Tw} \text { Ger }
$$

are also quasi-isomorphisms of dg operads.

As we will see below in Section 5.1. many objects of the under-category $\Lambda$ Lie $_{\infty} \downarrow$ Operads satisfy the following remarkable property:

Definition 5.3. An arrow $\Lambda \mathrm{Lie}_{\infty} \rightarrow \mathcal{O}$ is called a homotopy fixed point for $\mathrm{Tw}$ if the counit map $\eta_{\mathcal{O}}$ : $\operatorname{Tw} \mathcal{O} \rightarrow \mathcal{O}$ is a quasi-isomorphism.

If the map from $\Lambda \mathrm{Lie}_{\infty}$ is clear from the context, we will say, by abusing the notation, that $\mathcal{O}$ is a homotopy fixed point of $\mathrm{Tw}$.

Theorem 5.1 implies that, if a $\mathrm{dg}$ operad $\mathcal{O}$ is a homotopy fixed point for Tw and a $\mathrm{dg}$ operad $\mathcal{O}^{\prime}$ is quasi-isomorphic to $\mathcal{O}$, then $\mathcal{O}^{\prime}$ is also a homotopy fixed point for Tw . 
Example 5.4. In Subsection 5.1.1 below, we will show that the operads $\Lambda$ Lie and Ger are homotopy fixed points for $\mathrm{Tw}$. Hence, by Theorem 5.1, the $d g$ operads $\Lambda \mathrm{Lie}_{\infty}$ and $\mathrm{Ger}_{\infty}$ are also homotopy fixed points for $\mathrm{Tw}$.

Example 5.5. In Section 9.3 below, we will show that the $d g$ operad $\mathrm{Br}$ governing braces algebras (see Section (9) is a homotopy fixed point for $\mathrm{Tw}$.

5.1. The distributive law and the functor $\mathrm{Tw}$. In this section, we describe a large class of dg operads which are simultaneously $\mathrm{Tw}$-coalgebras and homotopy fixed points for $\mathrm{Tw}$.

Let us recall [14] that, for collections $P$ and $Q$ in $\mathfrak{C}$, the formula

$$
P \odot Q(n)=\bigoplus_{r \geq 0} P(r) \otimes_{S_{r}}\left(\bigoplus_{k_{1}+\cdots+k_{r}=n} \operatorname{Ind}_{S_{k_{1}} \times \cdots \times S_{k_{r}}}^{S_{n}} Q\left(k_{1}\right) \otimes \cdots \otimes Q\left(k_{r}\right)\right)
$$

defines a monoidal structure on the category of collections. The product (5.1) is known as plethysm.

Let $P$ be an arbitrary dg operad. Furthermore, let $\mathcal{O}$ be the collection of cochain complexes

$$
\mathcal{O}=P \odot \Lambda \text { Lie }
$$

which is obtained by computing the plethysm (5.1) of $P$ with $\Lambda$ Lie.

Let $\varsigma_{1, i}$ be the cycle $(1,2, \ldots, i)$ in $S_{n+1}$. It is not hard to see that imposing the relation

$$
\left\{a_{1}, a_{2}\right\} \circ_{2} \gamma=(-1)^{|\gamma|} \sum_{i=1}^{n} \varsigma_{1, i}\left(\gamma \circ_{i}\left\{a_{1}, a_{2}\right\}\right) \quad \forall \gamma \in P(n)
$$

and using the operad structures on $P$ and on $\Lambda$ Lie we get a natural dg operad structure on the collection (5.2). Following [24] and [22, Section 8.6], we say that the $\operatorname{dg}$ operad $\mathcal{O}$ is obtained from $P$ and $\Lambda$ Lie using the distributive law (5.3).

Example 5.6. The operad Ger is obtained from the operad Com via the above construction.

$$
\text { Ger }=\text { Com } \odot \Lambda \text { Lie . }
$$

For dg operads obtained in this way, we have the following straightforward proposition:

Proposition 5.7. Let $\mathcal{O}$ be the $d g$ operad which is obtained via taking the plethysm 5.2 and imposing relation (5.3). Then for every $\gamma \in \mathcal{O}(n)$ we have

$$
\left\{a_{1}, a_{2}\right\} \circ_{2} \gamma=(-1)^{|\gamma|} \sum_{i=1}^{n} \varsigma_{1, i}\left(\gamma \circ_{i}\left\{a_{1}, a_{2}\right\}\right) .
$$

Furthermore, for every $\mathcal{O}$-algebra $V$, the adjoint action $\{v$,$\} is a derivation of the \mathcal{O}$-algebra structure on $V$ for every $v \in V$.

Remark 5.8. Proposition 5.7 implies that the operad BV governing the Batalin-Vilkovisky (BV) algebra 12 is not an operad which is obtained via the above construction. Indeed, the unary operation $\delta$ on a $B V$ algebra $V$ satisfies the relation

$$
\delta\left(\left\{v_{1}, v_{2}\right\}\right)+\left\{\delta\left(v_{1}\right), v_{2}\right\}+(-1)^{\left|v_{1}\right|}\left\{v_{1}, \delta\left(v_{2}\right)\right\}=0 .
$$

Hence, in general, the adjoint action $\{v$,$\} is not a derivation of the B V$ algebra structure on $V$. 
Let $\mathcal{O}$ be the $d g$ operad which is obtained via taking the plethysm $(5.2)$ and imposing relation $(5.3)$. It is obvious that the $\mathrm{dg}$ operad $\mathcal{O}$ receives a natural embedding

$$
\mathfrak{i}: \Lambda \text { Lie } \hookrightarrow \mathcal{O}
$$

Composing $\mathfrak{i}$ with the canonical quasi-isomorphism $2.26 U_{\Lambda \text { Lie }}: \Lambda$ Lie $_{\infty} \rightarrow \Lambda$ Lie we get a map of dg operads

$$
\hat{\varphi}:=\mathfrak{i} \circ U_{\Lambda \text { Lie }}: \Lambda \text { Lie }_{\infty} \rightarrow \mathcal{O}
$$

Hence, we may apply the twisting procedure to the pair $(\mathcal{O}, \hat{\varphi})$ and obtain a $\operatorname{dg}$ operad $\operatorname{Tw} \mathcal{O}$.

According to Section 3.4 the spaces

$$
\mathrm{Tw}^{\oplus} \mathcal{O}(n)=\bigoplus \mathrm{s}^{2 r}(\mathcal{O}(r+n))^{S_{r}}
$$

form a sub- dg operad of $\mathrm{Tw} \mathcal{O}$.

It turns our that the $\mathrm{dg}$ operad $\mathrm{Tw}^{\oplus} \mathcal{O}$ coincides with $\mathrm{Tw} \mathcal{O}$, provided the $\mathrm{dg}$ operad $P$ satisfies a minor technical condition. Namely,

Proposition 5.9. Let $P$ be a dg operad for which there exists a integer $N$ such that for each $n \geq 0$ and for every $v \in P(n)$

$$
|v| \geq N
$$

If the $d g$ operad $\mathcal{O}$ is obtained via taking the plethysm 5.2 and imposing relation (5.3) then

$$
\mathrm{Tw}^{\oplus} \mathcal{O}=\operatorname{Tw} \mathcal{O}
$$

Proof. Let $m$ be an integer and $n$ be a non-negative integer. Our goal is to prove every sum

$$
\sum_{r=0}^{\infty} w_{r}, \quad w_{r} \in \mathbf{s}^{2 r}(\mathcal{O}(r+n))^{S_{r}}
$$

of a fixed degree $m$ has only finitely many terms.

For every $r \geq 0$, the graded vector space $\mathbf{s}^{2 r}(\mathcal{O}(r+n))^{S_{r}}$ is spanned by vectors of the form

$$
\sum_{\sigma \in S_{r}} \sigma\left(v ; X_{1}, X_{2}, \ldots, X_{t}\right),
$$

where $v \in P(t)$ for some $t$ and $X_{i}$ are vectors in $\Lambda \operatorname{Lie}\left(k_{i}\right)$ such that

$$
\sum_{i=1}^{t} k_{i}=n+r
$$

Since a vector 5.11 carries degree $m$ in

$$
\mathbf{s}^{2 r}(\mathcal{O}(r+n))^{S_{r}}
$$

we obtain the following equation

$$
m=2 r+|v|+\sum_{i=1}^{t}\left(1-k_{i}\right) .
$$

Combining 5.12 with 5.13 we get

$$
m=r+|v|+t-n
$$


and hence

$$
r=m+n-t-|v|
$$

Since $t \geq 0$ and $|v| \geq N$, we deduce that

$$
r \leq m+n-N
$$

Thus the sum in 5.10 has indeed only finitely many terms.

The following theorem is the central result of this section 10

Theorem 5.10. If an object $(\mathcal{O}, \hat{\varphi})$ of the under-category $\Lambda$ Lie $_{\infty} \downarrow$ Operads is obtained via taking the plethysm (5.2) of a dg operad $P$ with $\Lambda$ Lie and imposing relation $(5.3)$, then $(\mathcal{O}, \hat{\varphi})$ is canonically a Tw coalgebra. If, in addition, the $d g$ operad $P$ satisfies the condition of Proposition 5.9, then $\mathcal{O}$ is a homotopy fixed point for $\mathrm{Tw}$.

Proof. Identity (5.4) implies that the canonical embedding

$$
\begin{gathered}
\operatorname{emb}_{\mathcal{O}}: \mathcal{O} \rightarrow \operatorname{Tw} \mathcal{O} \\
\left(\operatorname{emb}_{\mathcal{O}}(v)\right)\left(1_{r}\right)= \begin{cases}v & \text { if } r=0, \\
0 & \text { otherwise }\end{cases}
\end{gathered}
$$

is compatible with the differentials $\partial^{\mathrm{Tw}}$ and $\partial^{\mathcal{O}}$. Furthermore, it is easy to see that the diagrams
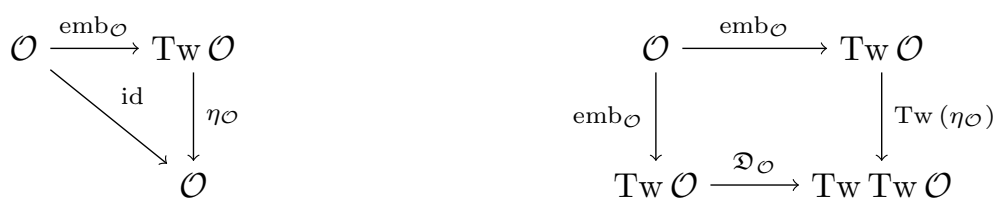

commute. Thus $(\mathcal{O}, \hat{\varphi})$ is indeed a coalgebra over the comonad Tw .

Let us now observe that the map emb $\operatorname{er}_{\mathcal{O}}$ lands into the sub- $\mathrm{dg}$ operad $\mathrm{Tw}^{\oplus} \mathcal{O}$. Furthermore, if the dg operad $P$ satisfies the condition of Proposition 5.9 , then

$$
\mathrm{Tw}^{\oplus} \mathcal{O}=\mathrm{Tw}_{\mathrm{O}}
$$

Thus, we need to prove that the map

$$
\left.\operatorname{emb}_{\mathcal{O}}\right|_{\mathcal{O}(n)}: \mathcal{O}(n) \hookrightarrow \mathrm{Tw}^{\oplus} \mathcal{O}(n)
$$

is a quasi-isomorphism of cochain complexes for every $n$. For this purpose we consider the free $\mathcal{O}$-algebra

$$
\mathcal{O}\left(a, a_{1}, a_{2}, \ldots, a_{n}\right)
$$

generated by $n$ dummy variables $a_{1}, a_{2}, \ldots, a_{n}$ of degree zero and one dummy variable $a$ of degree 2 . Next, we denote by $\delta$ the degree 1 derivation of the $\mathcal{O}$-algebra $\mathcal{O}\left(a, a_{1}, a_{2}, \ldots, a_{n}\right)$ defined by the formulas:

$$
\delta(a)=\frac{1}{2}\{a, a\}, \quad \delta\left(a_{i}\right)=0 \quad \forall 1 \leq i \leq n .
$$

\footnotetext{
${ }^{10}$ An idea of this proof is borrowed from 33 .
} 
Due to the Jacobi identity, we have $\delta^{2}=0$. Hence, $\delta$ is a differential on $\mathcal{O}$-algebra (5.19). We will combine $\delta$ with the differential $\partial^{\mathcal{O}}$ coming from $\mathcal{O}$ and consider the $\mathcal{O}$-algebra 5.19 with the differential

$$
\partial^{\mathcal{O}}+\delta
$$

Let us denote by

$$
\mathcal{O}^{\prime}\left(a, a_{1}, a_{2}, \ldots, a_{n}\right)
$$

the subcomplex of 5.19 which is spanned by $\mathcal{O}$-monomials in which each variable from the set $\left\{a_{1}, a_{2}, \ldots, a_{n}\right\}$ appears exactly once.

It is not hard to see that the formula

$$
\psi\left(\mathbf{s}^{2 r} v\right)=\frac{1}{r !} v \otimes a^{\otimes r} \otimes a_{1} \otimes a_{2} \otimes \cdots \otimes a_{n}, \quad v \in(\mathcal{O}(r+n))^{S_{r}}
$$

gives us an obvious isomorphism of graded vector spaces

$$
\psi: \mathrm{Tw}^{\oplus} \mathcal{O}(n)=\bigoplus_{r=0}^{\infty}(\mathcal{O}(r+n))^{S_{r}} \rightarrow \mathcal{O}^{\prime}\left(a, a_{1}, a_{2}, \ldots, a_{n}\right) .
$$

Using identity (5.4), one can show that $\psi$ intertwines the differentials $\partial^{\mathrm{Tw}}$ on $\mathrm{Tw}^{\oplus} \mathcal{O}(n)$ and 5.21 on (5.22). Hence, $\psi$ (5.24) is an isomorphism of cochain complexes.

Let us denote by

$$
\mathcal{O}^{\prime \prime}\left(a_{1}, a_{2}, \ldots, a_{n}\right)
$$

the subcomplex of the free $\mathcal{O}$-algebra $\mathcal{O}\left(a_{1}, a_{2}, \ldots, a_{n}\right)$ which is spanned by monomials in which each variable from the set $\left\{a_{1}, a_{2}, \ldots, a_{n}\right\}$ appears exactly once.

We observe that the assignment

$$
v \mapsto v \otimes a_{1} \otimes a_{2} \otimes \cdots \otimes a_{n}, \quad v \in \mathcal{O}(n)
$$

gives us an obvious identification between the cochain complex $\mathcal{O}(n)$ and 5.25 .

We also observe that the composition $\psi \circ \mathrm{emb}_{\mathcal{O}}$ operates by the formula

$$
\psi \circ \operatorname{emb}_{\mathcal{O}}(v)=v \otimes a_{1} \otimes a_{2} \otimes \cdots \otimes a_{n} .
$$

Thus, our goal is to show that the embedding

$$
\mathcal{O}^{\prime \prime}\left(a_{1}, a_{2}, \ldots, a_{n}\right) \hookrightarrow \mathcal{O}^{\prime}\left(a, a_{1}, a_{2}, \ldots, a_{n}\right)
$$

is a quasi-isomorphism of cochain complexes.

For this purpose we introduce the free $\Lambda$ Lie-algebra

$$
\Lambda \operatorname{Lie}\left(a, a_{1}, a_{2}, \ldots, a_{n}\right)
$$

generated by $n$ dummy variables $a_{1}, a_{2}, \ldots, a_{n}$ of degree zero and one dummy variable $a$ of degree 2 .

We consider the $\Lambda$ Lie-algebra $\Lambda \operatorname{Lie}\left(a, a_{1}, a_{2}, \ldots, a_{n}\right)$ with the differential $\delta$ defined in 5.20 .

In addition, we introduce two subspaces

$$
\Lambda \operatorname{Lie}^{\prime \prime}\left(a_{1}, a_{2}, \ldots, a_{n}\right) \subset \Lambda \operatorname{Lie}\left(a, a_{1}, a_{2}, \ldots, a_{n}\right)
$$

and

$$
\Lambda \operatorname{Lie}^{\prime}\left(a, a_{1}, a_{2}, \ldots, a_{n}\right) \subset \Lambda \operatorname{Lie}\left(a, a_{1}, a_{2}, \ldots, a_{n}\right) .
$$


Here $\Lambda \operatorname{Lie}^{\prime}\left(a, a_{1}, a_{2}, \ldots, a_{n}\right)$ is spanned by $\Lambda$ Lie-monomials in $\Lambda$ Lie $\left(a, a_{1}, a_{2}, \ldots, a_{n}\right)$ which involve each variable from the set $\left\{a_{1}, a_{2}, \ldots, a_{n}\right\}$ at most once, and $\Lambda \operatorname{Lie}^{\prime \prime}\left(a_{1}, a_{2}, \ldots, a_{n}\right)$ is spanned by $\Lambda$ Lie-monomials in $\Lambda \operatorname{Lie}^{\prime}\left(a, a_{1}, a_{2}, \ldots, a_{n}\right)$ which do not involve the variable $a$ at all.

It is clear that both subspaces $(5.29)$ and $(5.30)$ are subcomplexes of 5.28$)$. Moreover, the restriction of the differential $\delta$ to 5.29 is zero.

The proof of Theorem 5.10 is based on the following statement:

Lemma 5.11. The embedding

$$
\mathrm{emb}: \Lambda \operatorname{Lie}^{\prime \prime}\left(a_{1}, a_{2}, \ldots, a_{n}\right) \hookrightarrow \Lambda \operatorname{Lie}^{\prime}\left(a, a_{1}, a_{2}, \ldots, a_{n}\right)
$$

is a quasi-isomorphism of cochain complexes. In other words, for every cocycle $c \in \Lambda \operatorname{Lie}^{\prime}\left(a, a_{1}, a_{2}, \ldots, a_{n}\right)$, there exists a vector $c_{1} \in \Lambda \operatorname{Lie}^{\prime}\left(a, a_{1}, a_{2}, \ldots, a_{n}\right)$ such that

$$
c-\delta\left(c_{1}\right) \in \Lambda \operatorname{Lie}^{\prime \prime}\left(a_{1}, a_{2}, \ldots, a_{n}\right) .
$$

The proof of this lemma is somewhat technical so we give it in Subsection 5.1 .2 below. To deduce the desired statement from Lemma (5.11), we observe that the cone of the embedding (5.27) is a direct summand in the cone of the embedding

$$
P\left(\Lambda \operatorname{Lie}^{\prime \prime}\left(a_{1}, a_{2}, \ldots, a_{n}\right)\right) \hookrightarrow P\left(\Lambda \operatorname{Lie}^{\prime}\left(a, a_{1}, a_{2}, \ldots, a_{n}\right)\right) .
$$

Hence the map (5.27) is a quasi-isomorphism if so is the map 5.32).

To prove that the embedding (5.32) is a quasi-isomorphism we consider the following increasing filtration on the cochain complex $P\left(\Lambda \operatorname{Lie}^{\prime}\left(a, a_{1}, a_{2}, \ldots, a_{n}\right)\right)$ :

$$
\cdots \subset \mathcal{F}^{m} P\left(\Lambda \operatorname{Lie}^{\prime}\left(a, a_{1}, a_{2}, \ldots, a_{n}\right)\right) \subset \mathcal{F}^{m+1} P\left(\Lambda \operatorname{Lie}^{\prime}\left(a, a_{1}, a_{2}, \ldots, a_{n}\right)\right) \subset \ldots,
$$

where

$$
\mathcal{F}^{m} P\left(\Lambda \operatorname{Lie}^{\prime}\left(a, a_{1}, a_{2}, \ldots, a_{n}\right)\right.
$$

is spanned by $\mathcal{O}$-monomials $w$ for which

$$
\operatorname{deg}_{a}(w)-|w| \leq m
$$

with $\operatorname{deg}_{a}(w)$ being the degree of $w$ in $a$. Since the differential $\partial^{\mathcal{O}}$ does not change the degree in $a$ and the differential $\delta$ raises it by 1 the total differential $\partial^{\mathcal{O}}+\delta$ is compatible with the filtration (5.33). Restricting (5.33) to the subcomplex $P\left(\Lambda \operatorname{Lie}^{\prime \prime}\left(a_{1}, a_{2}, \ldots, a_{n}\right)\right)$ we get the "silly" filtration

$$
\mathcal{F}^{m} P\left(\Lambda \operatorname{Lie}^{\prime \prime}\left(a_{1}, a_{2}, \ldots, a_{n}\right)\right)^{k}=\left\{\begin{array}{l}
P\left(\Lambda \operatorname{Lie}^{\prime \prime}\left(a_{1}, a_{2}, \ldots, a_{n}\right)\right)^{k} \\
0 \quad \text { otherwise } k \geq-m,
\end{array}\right.
$$

with the zero differential on the associated graded complex.

To describe the associated graded complex for 5.33 we denote by $\widetilde{P}$ the operad in grVect $_{\mathbb{K}}$ which is obtained from $P$ by forgetting the differential. It is clear from the construction that the associated graded complex

$$
\operatorname{Gr} P\left(\Lambda \operatorname{Lie}^{\prime}\left(a, a_{1}, a_{2}, \ldots, a_{n}\right)\right)
$$

is isomorphic to the cochain complex

$$
\widetilde{P}\left(\Lambda \operatorname{Lie}^{\prime}\left(a, a_{1}, a_{2}, \ldots, a_{n}\right)\right)
$$


with the differential $\delta$. Using the Künneth theorem and the fact that cohomology commutes with taking coinvariants, we deduce from Lemma (5.11) that the map

$$
\left(\widetilde{P}(n) \otimes\left(\Lambda \operatorname{Lie}^{\prime \prime}\left(a_{1}, a_{2}, \ldots, a_{n}\right)\right)^{\otimes n}\right)_{S_{n}} \rightarrow\left(\widetilde{P}(n) \otimes\left(\Lambda \operatorname{Lie}^{\prime}\left(a, a_{1}, a_{2}, \ldots, a_{n}\right)\right)^{\otimes n}\right)_{S_{n}}
$$

is a quasi-isomorphism of cochain complexes for every $n$. Thus the embedding (5.32) induces a quasiisomorphism on the level of associated graded complexes. Combining this observation with the fact that the filtrations (5.33) and (5.34) are locally bounded and cocomplete, we deduce, from Lemma A.3 in [8, Appendix $\mathrm{A}]$, that the embedding (5.32) is also a quasi-isomorphism. Hence the map (5.27) is a quasi-isomorphism of cochain complexes and the theorem is proved.

5.1.1. The operads $\Lambda$ Lie, Ger, $\Lambda \mathrm{Lie}_{\infty}$, and $\mathrm{Ger}_{\infty}$ are homotopy fixed points for Tw . Theorem 5.10 has the following useful Corollary:

Corollary 5.12. The operads $\Lambda$ Lie and Ger carry a canonical Tw-coalgebra structure. Moreover, these operads are homotopy fixed points for $\mathrm{Tw}$.

Combining Corollary 5.12 with Theorem 5.1 , we immediately conclude that 11

Corollary 5.13. The $d g$ operads $\Lambda \mathrm{Lie}_{\infty}$ and $\mathrm{Ger}_{\infty}$ are homotopy fixed points for $\mathrm{Tw}$.

Remark 5.14. Let us recall that, due to Lemma 3.11 and Theorem 4.12, both dg operads $\Lambda$ Lie $_{\infty}$ and Ger $\infty$ carry canonical $\mathrm{Tw}$-coalgebra structures. In fact, the canonical map of operads

$$
\mathfrak{T}: \Lambda \mathrm{Lie}_{\infty} \rightarrow \mathrm{Tw} \Lambda \mathrm{Lie}_{\infty}
$$

was used to introduce the notion of $\mathrm{Tw}$-coalgebra.

5.1.2. Proof of Lemma 5.11. The proof of Theorem 5.10 was based on Lemma 5.11. Here we give a proof of this lemma. We consider a non-empty ordered subset $\left\{i_{1}<i_{2}<\cdots<i_{k}\right\}$ of $\{1,2, \ldots, n\}$ and denote by

$$
\Lambda \operatorname{Lie}^{\prime}\left(a, a_{i_{1}}, \ldots, a_{i_{k}}\right)
$$

the subcomplex of $\Lambda \operatorname{Lie}^{\prime}\left(a, a_{1}, \ldots, a_{n}\right)$ which is spanned by $\Lambda$ Lie-monomials in $\Lambda$ Lie $\left(a, a_{i_{1}}, \ldots, a_{i_{k}}\right)$ involving each variable in the set $\left\{a_{i_{1}}, \ldots, a_{i_{k}}\right\}$ exactly once.

It is clear that $\Lambda \operatorname{Lie}^{\prime}\left(a, a_{1}, \ldots, a_{n}\right)$ splits into the direct sum of subcomplexes:

$$
\Lambda \operatorname{Lie}^{\prime}\left(a, a_{1}, \ldots, a_{n}\right)=\mathbb{K}\langle a,\{a, a\}\rangle \oplus \underset{\left\{i_{1}<i_{2}<\cdots<i_{k}\right\}}{\bigoplus} \Lambda \operatorname{Lie}^{\prime}\left(a, a_{i_{1}}, \ldots, a_{i_{k}}\right),
$$

where the summation runs over all non-empty ordered subsets $\left\{i_{1}<i_{2}<\cdots<i_{k}\right\}$ of $\{1,2, \ldots, n\}$.

It is not hard to see that the subcomplex $\mathbb{K}\langle a,\{a, a\}\rangle$ is acyclic. Thus our goal is to show that every cocycle in $\Lambda \operatorname{Lie}^{\prime}\left(a, a_{i_{1}}, \ldots, a_{i_{k}}\right)$ is cohomologous to cocycle in the intersection

$$
\Lambda \operatorname{Lie}^{\prime}\left(a, a_{i_{1}}, \ldots, a_{i_{k}}\right) \cap \Lambda \operatorname{Lie}^{\prime \prime}\left(a_{1}, a_{2}, \ldots, a_{n}\right) .
$$

To prove this fact we consider the tensor algebra

$$
T\left(\mathbb{K}\left\langle\mathbf{s}^{-1} a, \mathbf{s}^{-1} a_{i_{1}}, \mathbf{s}^{-1} a_{i_{2}}, \ldots, \mathbf{s}^{-1} a_{i_{k-1}}\right\rangle\right)
$$

in the variables $\mathbf{s}^{-1} a, \mathbf{s}^{-1} a_{i_{1}}, \mathbf{s}^{-1} a_{i_{2}}, \ldots, \mathbf{s}^{-1} a_{i_{k-1}}$ and denote by

$$
T^{\prime}\left(\mathbf{s}^{-1} a, \mathbf{s}^{-1} a_{i_{1}}, \mathbf{s}^{-1} a_{i_{2}}, \ldots, \mathbf{s}^{-1} a_{i_{k-1}}\right)
$$

\footnotetext{
${ }^{11}$ The same result for the operad $\mathrm{Lie}_{\infty}$ was obtained in [5 Section 7] by J. Chuang and A. Lazarev.
} 
the subspace of 5.38 which is spanned by monomials involving each variable from the set

$$
\left\{\mathbf{s}^{-1} a_{i_{1}}, \mathbf{s}^{-1} a_{i_{2}}, \ldots, \mathbf{s}^{-1} a_{i_{k-1}}\right\}
$$

exactly once.

It is not hard to see that the formula

$$
\nu\left(x_{j_{1}} \otimes x_{j_{2}} \otimes \cdots \otimes x_{j_{N}}\right)=\left\{\mathbf{s} x_{j_{1}},\left\{\mathbf{s} x_{j_{2}},\left\{\ldots\left\{\mathbf{s} x_{j_{N}}, a_{i_{k}}\right\} . .\right\}\right.\right.
$$

defines an isomorphism of the graded vector spaces

$$
\nu: T^{\prime}\left(\mathbf{s}^{-1} a, \mathbf{s}^{-1} a_{i_{1}}, \mathbf{s}^{-1} a_{i_{2}}, \ldots, \mathbf{s}^{-1} a_{i_{k-1}}\right) \stackrel{\cong}{\cong} \Lambda \operatorname{Lie}^{\prime \prime}\left(a, a_{i_{1}}, \ldots, a_{i_{k}}\right) .
$$

Let us denote by $\delta_{T}$ a degree 1 derivation of the tensor algebra (5.38) defined by the equations

$$
\delta_{T}\left(\mathbf{s}^{-1} a_{i_{t}}\right)=0, \quad \delta_{T}\left(\mathbf{s}^{-1} a\right)=\mathbf{s}^{-1} a \otimes \mathbf{s}^{-1} a .
$$

It is not hard to see that $\left(\delta_{T}\right)^{2}=0$. Thus, $\delta_{T}$ is a differential on the tensor algebra (5.38).

The subspace $(5.39)$ is obviously a subcomplex of (5.38). Furthermore, using the following consequence of Jacobi identity

$$
\{a,\{a, X\}\}=-\frac{1}{2}\{\{a, a\}, X\}, \quad \forall X \in \Lambda \operatorname{Lie}\left(a, a_{1}, \ldots, a_{n}\right),
$$

it is easy to show that

$$
\delta \circ \nu=\nu \circ \delta_{T} .
$$

Thus $\nu$ is an isomorphism from the cochain complex

$$
\left(T^{\prime}\left(\mathbf{s}^{-1} a, \mathbf{s}^{-1} a_{i_{1}}, \mathbf{s}^{-1} a_{i_{2}}, \ldots, \mathbf{s}^{-1} a_{i_{k-1}}\right), \delta_{T}\right)
$$

to the cochain complex

$$
\left(\Lambda \operatorname{Lie}^{\prime}\left(a, a_{i_{1}}, \ldots, a_{i_{k}}\right), \delta\right) .
$$

To compute cohomology of the cochain complex

$$
\left(T\left(\mathbb{K}\left\langle\mathbf{s}^{-1} a, \mathbf{s}^{-1} a_{i_{1}}, \mathbf{s}^{-1} a_{i_{2}}, \ldots, \mathbf{s}^{-1} a_{i_{k-1}}\right\rangle\right), \delta_{T}\right)
$$

we observe that the truncated tensor algebra

$$
\underline{T}_{\mathbf{s}^{-1} a}:=\underline{T}\left(\mathbb{K}\left\langle\mathbf{s}^{-1} a\right\rangle\right)
$$

forms an acyclic subcomplex of 5.42.

We also observe that the cochain complex (5.42) splits into the direct sum of subcomplexes

$$
\begin{gathered}
T\left(\mathbb{K}\left\langle\mathbf{s}^{-1} a, \mathbf{s}^{-1} a_{i_{1}}, \mathbf{s}^{-1} a_{i_{2}}, \ldots, \mathbf{s}^{-1} a_{i_{k-1}}\right\rangle\right)=T\left(\mathbb{K}\left\langle\mathbf{s}^{-1} a_{i_{1}}, \mathbf{s}^{-1} a_{i_{2}}, \ldots, \mathbf{s}^{-1} a_{i_{k-1}}\right\rangle\right) \oplus \\
\bigoplus_{m \geq 2, p_{1}, \ldots, p_{m}} V_{a_{\bullet}}^{\otimes p_{1}} \otimes \underline{T}_{\mathbf{s}^{-1} a} \otimes V_{a_{\bullet}}^{\otimes p_{2}} \otimes \underline{T}_{\mathbf{s}^{-1} a} \otimes \cdots \otimes V_{a_{\bullet}}^{\otimes p_{m-1}} \otimes \underline{T}_{\mathbf{s}^{-1} a} \otimes V_{a_{\bullet}}^{\otimes p_{m}}
\end{gathered}
$$

where $V_{a}$ is the cochain complex

$$
V_{a}:=\mathbb{K}\left\langle\mathbf{s}^{-1} a_{i_{1}}, \mathbf{s}^{-1} a_{i_{2}}, \ldots, \mathbf{s}^{-1} a_{i_{k-1}}\right\rangle
$$

with the zero differential and the summation runs over all combinations $\left(p_{1}, \ldots, p_{m}\right)$ of integers satisfying the conditions

$$
p_{1}, p_{m} \geq 0, \quad p_{2}, \ldots, p_{m-1} \geq 1 .
$$

By Künneth's theorem all the subcomplexes

$$
V_{a_{\bullet}}^{\otimes p_{1}} \otimes \underline{T}_{\mathbf{s}^{-1} a} \otimes V_{a_{\bullet}}^{\otimes p_{2}} \otimes \underline{T}_{\mathbf{s}^{-1} a} \otimes \cdots \otimes V_{a_{\bullet}}^{\otimes p_{m-1}} \otimes \underline{T}_{\mathbf{s}^{-1} a} \otimes V_{a_{\bullet}}^{\otimes p_{m}}
$$


are acyclic. Hence for every cocycle $c$ in 5.42 there exists a vector $c_{1}$ in 5.42 such that

$$
c-\delta_{T}\left(c_{1}\right) \in T\left(\mathbb{K}\left\langle\mathbf{s}^{-1} a_{i_{1}}, \mathbf{s}^{-1} a_{i_{2}}, \ldots, \mathbf{s}^{-1} a_{i_{k-1}}\right\rangle\right) .
$$

Combining this observation with the fact that the subcomplex 5.39 is a direct summand in 5.42 , we conclude that, for every cocycle $c$ in (5.39) there exists a vector $c_{1}$ in (5.39) such that

$$
c-\delta_{T}\left(c_{1}\right) \in T^{\prime}\left(\mathbf{s}^{-1} a, \mathbf{s}^{-1} a_{i_{1}}, \mathbf{s}^{-1} a_{i_{2}}, \ldots, \mathbf{s}^{-1} a_{i_{k-1}}\right) \cap T\left(\mathbb{K}\left\langle\mathbf{s}^{-1} a_{i_{1}}, \mathbf{s}^{-1} a_{i_{2}}, \ldots, \mathbf{s}^{-1} a_{i_{k-1}}\right\rangle\right) .
$$

Since the map $\nu$ (5.40) is an isomorphism from the cochain complex 5.39) with the differential $\delta_{T}$ to the cochain complex 5.36) with the differential $\delta$, we deduce that every cocycle in 5.36 is cohomologous to a unique cocycle in the intersection

$$
\Lambda \operatorname{Lie}^{\prime}\left(a, a_{i_{1}}, \ldots, a_{i_{k}}\right) \cap \Lambda \operatorname{Lie}^{\prime \prime}\left(a_{1}, \ldots, a_{n}\right) .
$$

Therefore every cocycle in $\Lambda \operatorname{Lie}^{\prime}\left(a, a_{1}, \ldots, a_{n}\right)$ is cohomologous to a unique cocycle in the subcomplex

$$
\Lambda \operatorname{Lie}^{\prime \prime}\left(a_{1}, \ldots, a_{n}\right) .
$$

Lemma 5.11 is proved.

5.1.3. The operads $\mathrm{As}$ and $\mathrm{As}_{\infty}$ are $\mathrm{Tw}$-coalgebras and are homotopy fixed points for $\mathrm{Tw}$. Recall that $\mathrm{As}$ is the operad which governs associative algebras without unit.

We have the obvious map of operads

$$
\text { Lie } \rightarrow \text { As }
$$

and hence the maps of operads

$$
\mathfrak{i}: \Lambda \text { Lie } \rightarrow \Lambda \text { As }
$$

and

$$
\Lambda \mathrm{Lie}_{\infty} \rightarrow \Lambda \mathrm{As}
$$

In other words, the operad $\Lambda$ As is naturally an object of the under-category $\Lambda$ Lie $_{\infty} \downarrow$ Operads.

Let us show that

Proposition 5.15. The operad $\Lambda \mathrm{As}$ is naturally a $\mathrm{Tw}$-coalgebra. Furthermore, $\Lambda \mathrm{As}$ is a homotopy fixed point for the functor $\mathrm{Tw}$.

Remark 5.16. Before we proceed to the proof, we should remark that the associativity law for $\Lambda$ As-algebras has a "funny" sign factor. Namely, a $\Lambda$ As-algebra structure on a graded vector space $V$ is a degree -1 binary operation · on $V$ satisfying the associativity condition

$$
\left(v_{1} \cdot v_{2}\right) \cdot v_{3}=-(-1)^{\left|v_{1}\right|} v_{1} \cdot\left(v_{2} \cdot v_{3}\right) .
$$

Proof. Although the operad $\Lambda$ As is not obtained $\sqrt{12}$ via taking a plethysm of $\Lambda$ Lie with another operad, it is not hard to see that for every vector $v \in \Lambda \operatorname{As}(n)$

$$
\mathfrak{i}\left(\left\{a_{1}, a_{2}\right\}\right) \circ_{2} v=(-1)^{|v|} \sum_{i=1}^{n} \varsigma_{1, i}\left(v \circ_{i} \mathfrak{i}\left(\left\{a_{1}, a_{2}\right\}\right)\right),
$$

where $\varsigma_{1, i}$ is the cycle $(1,2, \ldots, i)$.

\footnotetext{
${ }^{12}$ Instead, $\Lambda$ As carries a natural filtration, such that $\operatorname{Gr} \Lambda \mathrm{As} \cong \Lambda$ Com $\odot \Lambda$ Lie.
} 
Using this identity, it is easy to see that the canonical embedding

$$
\begin{gathered}
\text { emb }: \Lambda \mathrm{As} \rightarrow \mathrm{Tw} \Lambda \mathrm{As} \\
(\operatorname{emb}(v))\left(1_{r}\right)= \begin{cases}v & \text { if } r=0, \\
0 & \text { otherwise }\end{cases}
\end{gathered}
$$

is compatible with the differentials, i.e.

$$
\partial^{\mathrm{Tw}}(\operatorname{emb}(v))=0, \quad \forall v \in \Lambda \mathrm{As}(n) .
$$

A straightforward verification shows that the map emb (5.48) satisfies the axioms of the Tw-coalgebra.

To show that $\Lambda \mathrm{As}$ is a homotopy fixed point for $\mathrm{Tw}$, we consider the free $\Lambda \mathrm{As}$-algebra $\Lambda \mathrm{As}\left(a, a_{1}, \ldots, a_{n}\right)$ in $n$ dummy variables $a_{1}, \ldots, a_{n}$ of degree 0 and one dummy variable $a$ of degree 2 .

The algebra $\Lambda \operatorname{As}\left(a, a_{1}, \ldots, a_{n}\right)$ carries the differential $\delta$ defined by the formulas:

$$
\delta(a)=a \cdot a, \quad \delta\left(a_{i}\right)=0, \quad \forall 1 \leq i \leq n .
$$

Next, we denote by $\Lambda \mathrm{As}^{\prime}\left(a, a_{1}, \ldots, a_{n}\right)$ the subcomplex of $\Lambda \mathrm{As}\left(a, a_{1}, \ldots, a_{n}\right)$ which is spanned by $\Lambda$ Asmonomials in which each variable from the set $\left\{a_{1}, \ldots, a_{n}\right\}$ appears exactly once. It is not hard to see that the cochain complexes

$$
\operatorname{Tw} \Lambda \operatorname{As}(n) \quad \text { and } \quad \Lambda \mathrm{As}^{\prime}\left(a, a_{1}, \ldots, a_{n}\right)
$$

are isomorphic and, moreover, the natural embedding

$$
\Lambda \mathrm{As}(n) \hookrightarrow \Lambda \mathrm{As}^{\prime}\left(a, a_{1}, \ldots, a_{n}\right)
$$

induces an isomorphism on the level of cohomology.

This observation implies that the embedding emb (5.48) is a quasi-isomorphism of dg operads. Hence $\Lambda \mathrm{As}$ is indeed a homotopy fixed point for the functor $\mathrm{Tw}$.

According to Section 3.5, we may ask the same questions about the operad As keeping in mind the canonical map Lie $\rightarrow$ As. For this case, Proposition 5.15 gives us the following obvious corollary:

Corollary 5.17. The operad As is naturally a $\mathrm{Tw}$-coalgebra. Furthermore, As is a homotopy fixed point for the functor $\mathrm{Tw}$.

Let us denote by $\mathrm{As}_{\infty}$ the dg operad which governs $A_{\infty}$-algebras, i.e.

$$
\operatorname{As}_{\infty}=\operatorname{Cobar}(\Lambda \text { coAs }) .
$$

We claim that

Corollary 5.18. The $d g$ operad $\mathrm{As}_{\infty}$ is naturally a $\mathrm{Tw}$-coalgebra. Furthermore, $\mathrm{As}_{\infty}$ is a homotopy fixed point for the functor $\mathrm{Tw}$.

Proof. The second claim is an obvious consequence of Theorem 5.1 and Corollary 5.17. So it remains to prove the first claim.

Because of sign factor, it is more convenient to prove that the dg operad

$$
\Lambda^{-1} \mathrm{As}_{\infty}=\operatorname{Cobar}(\mathrm{coAs})
$$

is a Tw-coalgebra. Then the desired statement will follow from the arguments of Section 3.5 
Our goal is to produce a map of dg operads

$$
c: \operatorname{Cobar}(\mathrm{coAs}) \rightarrow \mathrm{Tw} \text { Cobar }(\mathrm{coAs})
$$

and verify axioms of the coalgebra over $\mathrm{Tw}$.

For this purpose we recall that $\operatorname{As}(n)$ has the canonical basis

$$
\left\{a_{\sigma(1)} a_{\sigma(2)} \ldots a_{\sigma(n)}\right\}_{\sigma \in S_{n}},
$$

where $a_{1}, a_{2}, \ldots, a_{n}$ are dummy variables of degree zero.

Next, we denote by $\left(a_{\sigma(1)} a_{\sigma(2)} \ldots a_{\sigma(n)}\right)^{*}$ vectors of the dual basis in $\operatorname{coAs}(n)=(\operatorname{As}(n))^{*}$ and observe that the formula $(n \geq 2, r \geq 0)$

$$
\alpha\left(\left(a_{\sigma(1)} a_{\sigma(2)} \ldots a_{\sigma(n)}\right)^{*}\right)\left(1_{r}\right)=\sum_{\lambda \in \mathrm{Sh}_{r, n}} \sum_{\tau \in S_{r}} \mathbf{s} \lambda\left(a_{\tau(1)} \ldots a_{\tau(r)} a_{r+\sigma(1)} a_{r+\sigma(2)} \ldots a_{r+\sigma(n)}\right)^{*}
$$

defines a degree 1 vector in the dg Lie algebra $\operatorname{Conv}\left(\operatorname{coAs}_{\circ}\right.$, Tw Cobar $\left.(\operatorname{coAs})\right)$. Here

$$
\mathbf{s} \lambda\left(a_{\tau(1)} \ldots a_{\tau(r)} a_{r+\sigma(1)} a_{r+\sigma(2)} \ldots a_{r+\sigma(n)}\right)^{*}
$$

is viewed as a vector in $\mathbf{s} \operatorname{coAs}(r+n) \subset \operatorname{Cobar}(\operatorname{coAs})$.

A direct computation shows that $\alpha$ is a Maurer-Cartan element of the dg Lie algebra

$$
\operatorname{Conv}\left(\operatorname{coAs} s_{\circ}, \operatorname{Tw} \operatorname{Cobar}(\operatorname{coAs})\right)
$$

Hence, by Theorem 2.2 $\alpha$ gives us a map of dg operads 5.52 .

It is easy to check that this map satisfies all the axioms of the coalgebra over the comonad Tw. So we leave the verification of these axioms to the reader.

Remark 5.19. In Section 7 of [5], J. Chuang and A. Lazarev also proved that As and $\mathrm{As}_{\infty}$ are homotopy fixed points for the functor $\mathrm{Tw}$.

5.1.4. Example: a $\mathrm{Tw}$-coalgebra which is not a homotopy fixed point for $\mathrm{Tw}$. We constructed a large class of $\mathrm{Tw}$-coalgebras each of which is a homotopy fixed point for $\mathrm{Tw}$. Let us now give an example of a Tw coalgebra which is not a homotopy fixed point for Tw . Let $P$ be an operad in the category grVect $_{\mathbb{K}}$ such that $P(n) \neq 0$ for all $n \geq 1$. (For example, the operad As would work.) Let

$$
\hat{\varphi}: \Lambda \mathrm{Lie}_{\infty} \rightarrow P
$$

be the zero map. Then the $\operatorname{dg}$ operads Tw $P$ and $\operatorname{Tw}(\mathrm{Tw} P)$ with the spaces

$$
\operatorname{Tw} P(n)=\prod_{r \geq 0} \mathbf{s}^{2 r}(P(r+n))^{S_{r}}
$$

and

$$
\operatorname{Tw}(\operatorname{Tw} P)(n)=\prod_{r, s \geq 0} \mathbf{s}^{2 r+2 s}(P(r+s+n))^{S_{r} \times S_{s}}
$$

carry the zero differentials. The operad $\mathrm{Tw} P$ is a Tw-coalgebra by construction, however,

Proposition 5.20. The operad $\mathrm{Tw} P$ is not a homotopy fixed point for Tw . 
Proof. Let $r, n, s$ be a positive integers and let $v$ be a non-zero vector in $P(r+s+n)$. Such a vector exists, since $P(n) \neq 0$ for all $n \geq 1$. The sum

$$
\sum_{\sigma \in S_{r} \times S_{s}} \mathbf{s}^{2 r+2 s} \sigma(v)
$$

can be viewed as a vector in $\operatorname{Tw}(\operatorname{Tw} P)(n)$. Since the differential on $\operatorname{Tw}(\operatorname{Tw} P)$ is non-zero, then vector (5.57) is a non-trivial cocycle. On the other hand, since $r \geq 1$, the counit map

$$
\eta_{\mathrm{Tw} P}: \operatorname{Tw}(\mathrm{Tw} P) \rightarrow \mathrm{Tw} P
$$

sends vector (5.57) to zero. Thus $\eta_{\mathrm{Tw} P}$ is not a quasi-isomorphism.

\section{Twisting $\mathcal{O}$-algebra structures by Maurer-Cartan elements}

Let $\mathcal{O}$ be an object of the under-category $\Lambda$ Lie $e_{\infty} \downarrow$ Operads. Following Section 3.2 we consider the category $\operatorname{Alg}_{\mathcal{O}}^{\mathrm{MC}}$ whose objects are pairs

$$
(V, \alpha)
$$

where $V$ is an $\mathcal{O}$-algebra equipped with a complete descending filtration ${ }^{13}$ and $\alpha \in \mathcal{F}_{1} V^{2}$ is a Maurer-Cartan element. Morphisms in $\operatorname{Alg}_{\mathcal{O}}^{\mathrm{MC}}$ are morphisms of filtered $\mathcal{O}$-algebras $f: V \rightarrow V^{\prime}$ which send $\alpha$ to $\alpha^{\prime}$.

Recall that Theorem 3.8 yields a functor

$$
\mathfrak{F}: \operatorname{Alg}_{\mathcal{O}}^{\mathrm{MC}} \rightarrow \operatorname{Alg}_{\mathrm{Tw} \mathcal{O}}^{\mathrm{filtr}}
$$

from the category $\operatorname{Alg}_{\mathcal{O}}^{\mathrm{MC}}$ to the category $\operatorname{Alg}_{\mathrm{Tw} \mathcal{O}}^{\mathrm{filtr}}$ of filtered $\mathrm{Tw} \mathcal{O}$-algebras. More precisely, the functor $\mathfrak{F}$ assigns to a pair $(V, \alpha)$ the cochain complex $V^{\alpha}$ with the differential $\partial^{\alpha} 3.22$ and the Tw $\mathcal{O}$-algebra structure defined by equation 3.23.

If the arrow $\hat{\varphi}: \Lambda \mathrm{Lie}_{\infty} \rightarrow \mathcal{O}$ is a $\mathrm{Tw}$-coalgebra then we have a morphism of dg operads

$$
c: \mathcal{O} \rightarrow \operatorname{Tw} \mathcal{O}
$$

which induces a functor

$$
c^{*}: \operatorname{Alg}_{\mathrm{Tw} \mathcal{O}}^{\mathrm{filtr}} \rightarrow \operatorname{Alg}_{\mathcal{O}}
$$

from the category $\operatorname{Alg}_{\mathrm{Tw} \mathcal{O}}^{\text {filtr }}$ to the category $\mathrm{Alg}_{\mathcal{O}}$ of $\mathcal{O}$-algebras.

Composing 6.1 and 6.3 we get the functor

$$
\mathrm{ATw}:=c^{*} \circ \mathfrak{F}: \operatorname{Alg}_{\mathcal{O}}^{\mathrm{MC}} \rightarrow \operatorname{Alg}_{\mathcal{O}} .
$$

As a cochain complex $\operatorname{ATw}(V, \alpha)$ is $V^{\alpha}$ with the differential $\partial^{\alpha} 3.22$ and the $\mathcal{O}$-algebra structure is induced by the map $c: \mathcal{O} \rightarrow \operatorname{Tw} \mathcal{O}$.

For a pair $(V, \alpha) \in \operatorname{Alg}_{\mathcal{O}}^{\mathrm{MC}}$, we say that the $\mathcal{O}$-algebra $\operatorname{ATw}(V, \alpha)$ is obtained from $V$ via twisting by a Maurer-Cartan element $\alpha$. We refer to the construction of $\operatorname{ATw}(V, \alpha)$ as the twisting procedur $\biguplus^{14}$ for $\mathcal{O}$-algebras.

Axioms of a coalgebra over the comonad Tw listed in Section 4.2 imply that the twisting procedure satisfies the following properties:

P1 Considered as the $\Lambda \operatorname{Lie}_{\infty}$-algebra, $\operatorname{ATw}(V, \alpha)$ is obtained via twisting the $\Lambda$ Lie $_{\infty}$ algebra $V$ by the Maurer-Cartan element $\alpha$.

\footnotetext{
${ }^{13}$ As above, we assume that the $\mathcal{O}$-algebra structure on $V$ is compatible with the filtration 3.2 .
}

${ }^{14}$ Note that this twisting procedure for $\mathcal{O}$-algebras is defined only if the dg operad $\mathcal{O}$ is a coalgebra over the comonad Tw . 
P2 Twisting by the zero Maurer-Cartan element $\alpha=0$ does not change the $\mathcal{O}$-algebra, i.e., $\operatorname{ATw}(V, 0)=$ $V$.

P3 If $\alpha$ is a Maurer-Cartan element of $V$ and $\alpha^{\prime}$ is a Maurer-Cartan element of $\operatorname{ATw}(V, \alpha)$ then the twisted $\mathcal{O}$-algebras

$$
\operatorname{ATw}\left(\operatorname{ATw}(V, \alpha), \alpha^{\prime}\right) \quad \text { and } \quad \operatorname{ATw}\left(V, \alpha+\alpha^{\prime}\right)
$$

coincide.

Indeed, Property $\mathbf{P} 1$ follows easily from commutativity of diagram 4.12).

Equation (3.23) implies that, if $\alpha=0$, then the Tw $\mathcal{O}$-algebra structure on $V^{\alpha}=V$ factors through the counit map $\eta_{\mathcal{O}}: \operatorname{Tw} \mathcal{O} \rightarrow \mathcal{O}$. Thus Property $\mathbf{P 2}$ holds because the composition 4.13 is the identity map on $\mathcal{O}$.

Finally, Property P3 follows from commutativity of diagram (4.14).

Let us now consider functorial properties of ATw with respect to morphisms in the under-category $\Lambda$ Lie $_{\infty} \downarrow$ Operads .

For any arrow $\Psi: \mathcal{O} \rightarrow \mathcal{O}^{\prime}$ which fits into the commutative diagram

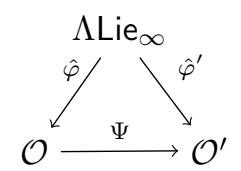

we can obviously extend the functor

$$
\Psi^{*}: \operatorname{Alg}_{\mathcal{O}^{\prime}} \rightarrow \operatorname{Alg}_{\mathcal{O}}
$$

to

$$
\Psi^{*}: \operatorname{Alg}_{\mathcal{O}^{\prime}}^{\mathrm{MC}} \rightarrow \mathrm{Alg}_{\mathcal{O}}^{\mathrm{MC}}
$$

Furthermore, we observe that the diagram

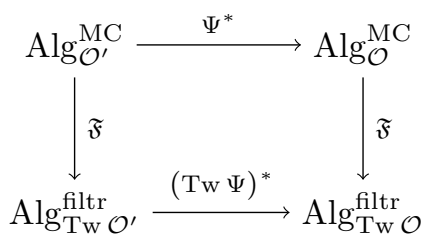

commutes because the construction of the functor $\mathfrak{F}(3.29)$ is functorial in $\mathcal{O}$.

If the dg operads $\mathcal{O}$ and $\mathcal{O}^{\prime}$ are Tw-coalgebras and the map $\Psi$ is compatible with the Tw-coalgebra structures then the diagram

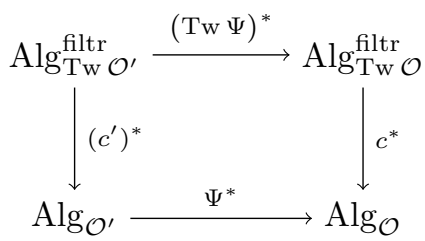

commutes. 
Thus, combining commutative diagrams 6.7 and 6.8 we arrive at the following functorial property of the twisting procedure.

Theorem 6.1. If $\Psi$ is a map of $\mathrm{Tw}$-coalgebras $\mathcal{O}$ and $\mathcal{O}^{\prime}$ then the diagram

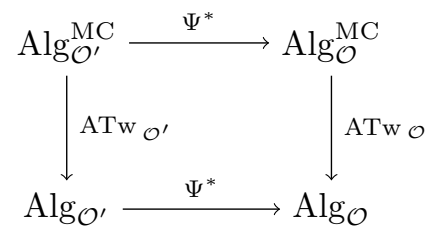

commutes.

At the end of this section, we observe that, under a mild technical condition, the functor ATw preserves quasi-isomorphisms. More precisely,

Proposition 6.2. Let $\mathcal{O}$ be a dg operad equipped with a Tw -coalgebra structure and let $f:(V, \alpha) \rightarrow\left(V^{\prime}, \alpha^{\prime}\right)$ be a morphism in $\mathrm{Alg}_{\mathcal{O}}^{\mathrm{MC}}$. Let us assume that the filtrations on $V$ and $V^{\prime}$ are bounded above. If $f$ is a quasiisomorphism of the underlying cochain complexes $(V, \partial)$ and $\left(V^{\prime}, \partial^{\prime}\right)$ then $f$ is also a quasi-isomorphism from $\operatorname{ATw}(V, \alpha)$ to $\operatorname{ATw}\left(V^{\prime}, \alpha^{\prime}\right)$.

Proof. The cone

$$
C=V^{\alpha} \oplus \mathbf{s}\left(V^{\prime}\right)^{\alpha^{\prime}}
$$

of the morphism $f: V^{\alpha} \rightarrow\left(V^{\prime}\right)^{\alpha^{\prime}}$ carries the obvious descending filtration

$$
\mathcal{F}_{m} C:=\mathcal{F}_{m} V^{\alpha} \oplus \mathbf{s} \mathcal{F}_{m}\left(V^{\prime}\right)^{\alpha^{\prime}}
$$

This filtration is complete and bounded above.

Furthermore, the associated graded complex

$$
\operatorname{Gr} C
$$

is isomorphic to the cone

$$
V \oplus \mathbf{s} V^{\prime}
$$

of the morphism $f: V \rightarrow V^{\prime}$.

Hence $\operatorname{Gr} C$ is acyclic and, by Lemma D.1 the cone of the morphism $f: V^{\alpha} \rightarrow\left(V^{\prime}\right)^{\alpha^{\prime}}$ is also acyclic.

Proposition 6.2 follows.

\section{ThE OPERAD OF BRACE TREES BT}

The remaining four sections of the paper are devoted to an application of the developed machinery to Deligne's conjecture. We will introduce an auxiliary operad BT, define the braces operad Br as a suboperad of Tw BT, and finally, prove Theorem 1.1 stated in the Introduction.

Let us start by defining the operad of brace trees BT.

To define the space $\mathrm{BT}(n)$ we introduce an auxiliary set $\mathcal{T}(n)$. An element of this set is a planar tree $T$ equipped with a bijection between $\{1,2, \ldots, n\}$ and the set

$$
V(T) \backslash\{\text { root vertex }\}
$$

of non-root vertices. For $n=0$ the set $\mathcal{T}(n)$ is empty. 
We call elements of $\mathcal{T}(n)$ brace trees. Examples of brace trees are shown on figures 7.1 and 7.2 . On figures,
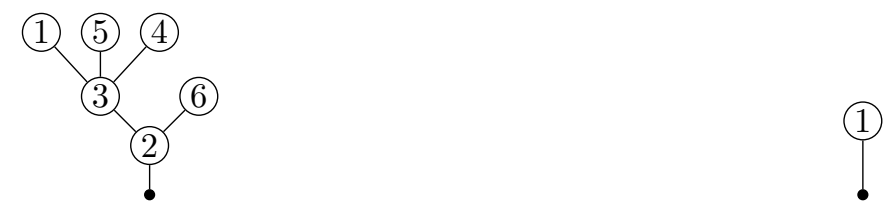

FIG. 7.1. A brace tree $T^{\prime} \in \mathcal{T}(6)$

FIG. 7.2. The brace tree $T_{\mathrm{id}} \in \mathcal{T}(1)$

non-root vertices are depicted by white circles with the corresponding numbers inscribed.

The $n$-th space $\mathrm{BT}(n)$ of BT consists of linear combinations of elements in $\mathcal{T}(n)$. The structure of a graded vector space on BT $(n)$ is obtained by declaring that each non-root edge carries degree -1 . In other words, for every $T \in \mathcal{T}(n)$ we have

$$
|T|=1-|E(T)|
$$

where $E(T)$ is the set of all edges of $T$. A simple combinatorics shows that for every brace tree $T \in \mathcal{T}(n)$ we have

$$
|T|=1-n
$$

Hence, the graded vector space $\mathrm{BT}(n)$ is concentrated in the single degree $1-n$. (In particular, the operad BT may only carry the zero differential.)

Since $\mathcal{T}(0)$ is empty, we have

$$
\mathrm{BT}(0)=\mathbf{0}
$$

Furthermore, since in $\mathcal{T}(1)$ we have only element $T_{\text {id }}$ (see figure 7.2 ,

$$
\mathrm{BT}(1)=\mathbb{K}
$$

7.1. The operad structure on BT. Let $T \in \mathcal{T}(n), T^{\prime} \in \mathcal{T}(k)$ and $1 \leq i \leq k$. Our goal is to define the output of the elementary insertion $T^{\prime} \circ_{i} T \in \mathrm{BT}(n+k-1)$.

Let $v_{i}$ be the non-root vertex of $T^{\prime}$ with label $i$. If $v_{i}$ is a leaf (i.e. $v_{i}$ does not have incoming edges) then the vector $T^{\prime} \circ_{i} T \in \mathrm{BT}(n+k-1)$ is, up to sign, represented by a brace tree $T^{\prime \prime}$ which is obtained from $T^{\prime}$ by erasing the vertex $v_{i}$ and gluing the brace tree $T$ via identifying the root edge of $T$ with the edge originating at $v_{i}$. After this operation we relabel elements of the set

$$
V\left(T^{\prime \prime}\right) \backslash\{\text { root vertex }\}=(V(T) \backslash\{\text { root vertex }\}) \sqcup\left(V\left(T^{\prime}\right) \backslash\left\{v_{i}\right\}\right)
$$

in the obvious way. The sign factor in front of $T^{\prime \prime}$ is obtained by keeping track of the reordering of non-root edges of $T^{\prime}$ and $T$.

Let us now consider the case when $v_{i}$ has $q \geq 1$ incoming edges. Since $T^{\prime}$ is a planar tree, these $q$ incoming edges are totally ordered. So we denote them by $e_{1}, e_{2}, \ldots, e_{q}$ keeping in mind that

$$
e_{1}<e_{2}<\cdots<e_{q} \text {. }
$$

The desired vector $T^{\prime} \circ_{i} T \in \mathrm{BT}(n+k-1)$ is represented by the sum

$$
T^{\prime} \circ_{i} T=\sum_{\alpha}(-1)^{f(\alpha)} T_{\alpha}
$$

where $T_{\alpha}$ is obtained from $T^{\prime}$ and $T$ following these steps: 
- first, we erase the vertex $v_{i}$ and glue the brace tree $T$ via identifying the root edge $T$ with the edge originating from $v_{i}$

- second, we attach the edges $e_{1}, e_{2}, \ldots, e_{q}$ to vertices in the set

$$
V(T) \backslash\{\text { root vertex }\}
$$

- finally, we relabel elements of the set

$$
V\left(T^{\prime \prime}\right) \backslash\{\text { root vertex }\}=(V(T) \backslash\{\text { root vertex }\}) \sqcup\left(V\left(T^{\prime}\right) \backslash\left\{v_{i}, \text { root vertex }\right\}\right)
$$

in the obvious way.

Ways of connecting the edges $e_{1}, e_{2}, \ldots, e_{q}$ to vertices in the set (7.7) should satisfy the following condition

Condition 7.1. The restriction of the total order on the set $E\left(T_{\alpha}\right)$ of $T_{\alpha}$ to the subset $\left\{e_{1}, e_{2}, \ldots, e_{q}\right\}$ should coincide with the order 7.5 .

This condition can be reformulated in geometric terms as follows. If we choose a small tubular neighborhood of the tree $T$ (drawn on the plane) and walk along its boundary starting from the root vertex in the clockwise direction then we will cross the edges in this order: first, we will cross $e_{1}$, second, we will cross $e_{2}$, third, we will cross $e_{3}$, and so on.

The summation in (7.6) goes over all ways $\alpha$ of connecting the edges $e_{1}, e_{2}, \ldots, e_{q}$ to vertices in the set 7.7) satisfying Condition 7.1

To define the sign factors $(-1)^{f(\alpha)}$ in $(7.6)$ we extend the total orders on the sets $E\left(T^{\prime}\right)$ and $(E(T) \backslash$ \{root edge\}) to the disjoint union

$$
E\left(T^{\prime}\right) \sqcup(E(T) \backslash\{\text { root edge }\})
$$

by declaring that all elements of $E\left(T^{\prime}\right)$ are smaller than elements of $E(T) \backslash\{$ root edge .

Next we observe that the set $(7.8)$ is naturally isomorphic to the set $E\left(T_{\alpha}\right)$ of edges of $T_{\alpha}$. On the other hand, the set $E\left(T_{\alpha}\right)$ carries a possibly different total order coming from planar structure on $T_{\alpha}$.

So the factor $(-1)^{f(\alpha)}$ is the sign of the permutation which connects these total orders on the set

$$
E\left(T_{\alpha}\right) \cong E\left(T^{\prime}\right) \sqcup(E(T) \backslash\{\text { root edge }\}) .
$$

Example 7.2. Let $T^{\prime}$ (resp. $T_{\circ \circ}$ ) be the brace tree depicted on figure 7.1 (resp. figure 7.3). The result of the insertion $T^{\prime} \mathrm{O}_{2} T$ is the sum of brace trees shown on figure 7.4.

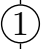

FIG. 7.3. The brace tree $T_{\circ \circ} \in \mathcal{T}(2)$

The symmetric group $S_{n}$ acts on $\mathrm{BT}(n)$ in the obvious way by rearranging the labels. It is not hard to see that operations (7.6) together with this action give us an operad structure on BT with the identity element represented by the brace tree $T_{\mathrm{id}}$ depicted on figure 7.2 


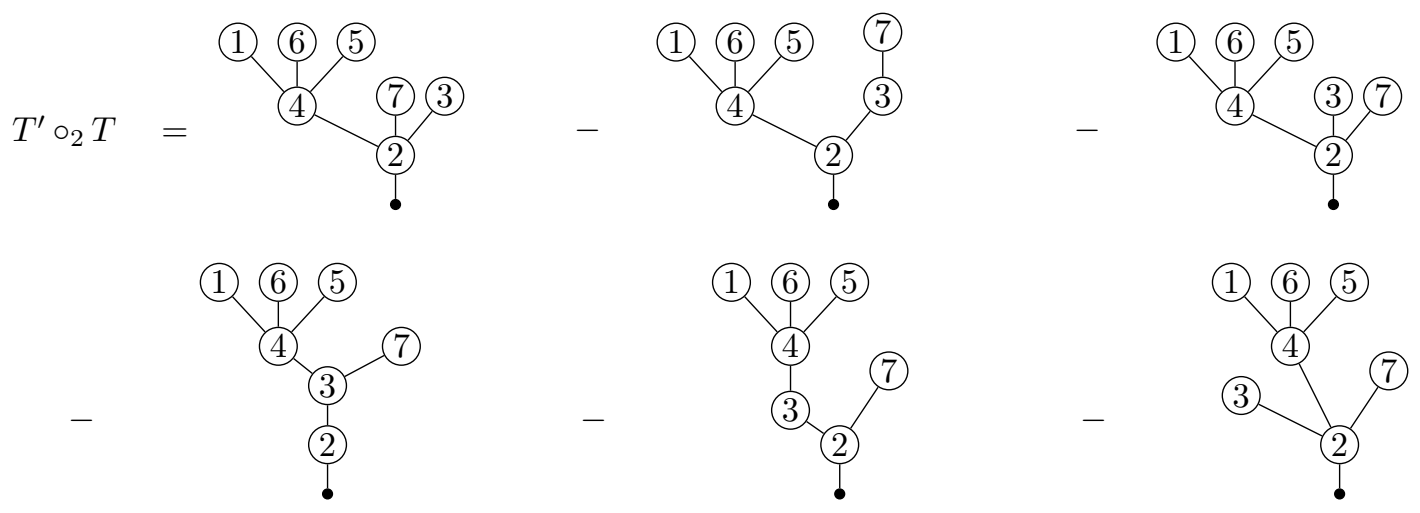

FIG. 7.4. The vector $T^{\prime} \circ_{2} T \in \mathrm{BT}(7)$

\section{The operad Tw BT}

The operad BT receives a natural map from $\Lambda$ Lie

$$
\varphi: \Lambda \text { Lie } \rightarrow \text { BT } .
$$

Since the operad $\Lambda$ Lie is generated by the binary bracket operation $\{\cdot, \cdot\} \in \Lambda \operatorname{Lie}(2)$, the map (8.1) is uniquely determined by its value $\varphi(\{\cdot, \cdot\})$, which equals

$$
\varphi(\{\cdot, \cdot\})=T_{\circ \circ}+\sigma_{12} T_{\circ \circ},
$$

where $T_{\circ \circ}$ is the brace tree depicted on figure 7.3 and $\sigma_{12}$ is the transposition in $S_{2}$. The desired compatibility with the Jacobi relation

$$
\varphi(\{\cdot, \cdot\}) \circ_{1} \varphi(\{\cdot, \cdot\})+\text { cyclic permutations }(1,2,3)=0
$$

can be checked by a straightforward computation.

In this section we give an explicit description for the twisted version Tw BT of BT corresponding to the map 8.2. As a graded vector space,

$$
\operatorname{Tw} \mathrm{BT}(n)=\prod_{r=0}^{\infty} \mathrm{s}^{2 r}(\mathrm{BT}(r+n))^{S_{r}} .
$$

Using the observation that for every $m$ the space $\mathrm{BT}(m)$ is concentrated in the single degree $1-m$ we conclude that the subspace Tw $\mathrm{BT}^{p}(n)$ of degree $p$ vectors in $\mathrm{Tw} \mathrm{BT}(n)$ is spanned by vectors of the form

$$
\sum_{\sigma \in S_{r}} \sigma(T)
$$

where $T$ is an arbitrary brace tree in $\mathcal{T}(r+n)$ and $r=p+n-1$. In particular,

$$
\mathrm{Tw} \mathrm{BT}=\mathrm{Tw}^{\oplus} \mathrm{BT}
$$

(cf. Section 3.4).

To represent vectors 8.5 , it is convenient to extend the set $\mathcal{T}(n)$ to another auxiliary set $\mathcal{T}^{\mathrm{tw}}(n)$. An element of $\mathcal{T}^{\text {tw }}(n)$ is a planar tree $T$ equipped with the following data:

- a partition of the set $V(T)$ of vertices

$$
V(T)=V_{\text {lab }}(T) \sqcup V_{\nu}(T) \sqcup V_{\text {root }}(T)
$$


into the singleton $V_{\text {root }}(T)$ consisting of the root vertex, the set $V_{\text {lab }}(T)$ consisting of $n$ vertices, and the set $V_{\nu}(T)$ consisting of vertices which we call neutral;

- a bijection between the set $V_{\text {lab }}(T)$ and the set $\{1,2, \ldots, n\}$;

We also call elements of $\mathcal{T}^{\mathrm{tw}}(n)$ brace trees. Figures $7.3,8.1,8.2$ show examples of brace trees in $\mathcal{T}^{\mathrm{tw}}(2)$. Figures 8.3 and 8.4 show examples of a brace tree in $\mathcal{T}^{\text {tw }}(1)$. On figures, neutral vertices of a brace

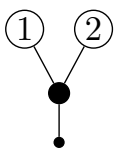

FiG. 8.1. A brace tree $T_{\cup} \in \mathcal{T}^{\mathrm{tw}}(2)$

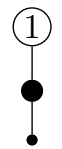

FIG. 8.3. A brace tree $T_{\bullet 1} \in \mathcal{T}^{\mathrm{tw}}(1)$

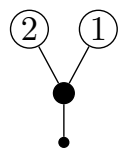

FIG. 8.2. A brace tree $T_{\cup o p p} \in \mathcal{T}^{\mathrm{tw}}(2)$

FIG. 8.4. A brace tree $T_{1} \bullet \in \mathcal{T}^{\mathrm{tw}}(1)$

tree in $\mathcal{T}^{\text {tw }}(n)$ are depicted by black circles and vertices in $V_{\text {lab }}(T)$ are depicted by white circles with the corresponding numbers inscribed.

We have the obvious bijection between brace trees in $\mathcal{T}^{\text {tw }}(n)$ with $r$ neutral vertices and linear combinations (8.5). This bijection assigns to a brace tree $T^{\prime}$ with $r$ neutral vertices the linear combination (8.5) where $T$ is obtained from $T^{\prime}$ by labeling the neutral vertices by $1,2, \ldots, r$ in any possible way and shifting the labels for vertices in $V_{\text {lab }}(T)$ up by $r$.

In virtue of this bijection, the $n$-th space Tw $\mathrm{BT}(n)$ of Tw BT is the space of (finite) linear combinations of brace trees in $\mathcal{T}^{\mathrm{tw}}(n)$. Furthermore, each brace tree $T^{\prime} \in \mathcal{T}^{\mathrm{tw}}(n)$ carries the degree

$$
|T|=2\left|V_{\nu}(T)\right|-|E(T)|+1,
$$

where $E(T)$ is the set of all edges of $T$. In other words, each non-root edge carries degree -1 and each neutral vertex carries degree 2 .

Using this description of Tw BT it is easy to define the elementary insertions in terms of brace trees. Indeed, let $T \in \mathcal{T}^{\text {tw }}(n), T^{\prime} \in \mathcal{T}^{\text {tw }}(k), 1 \leq i \leq k$ and let $v_{i}$ be the vertex in $V_{\text {lab }}\left(T^{\prime}\right)$ with label $i$. If $v_{i}$ is a leaf (i.e. $v_{i}$ does not have incoming edges) then the vector $T^{\prime} \circ_{i} T \in \mathrm{Tw} \mathrm{BT}(n+k-1)$ is represented by a tree $T^{\prime \prime}$ which is obtained from $T^{\prime}$ by erasing the vertex $v_{i}$ and gluing the tree $T$ via identifying the root edge of $T$ with the edge originating at $v_{i}$. After this operation we relabel elements of the set $V_{\text {lab }}\left(T^{\prime \prime}\right)=V_{\text {lab }}(T) \sqcup\left(V_{\text {lab }}\left(T^{\prime}\right) \backslash\left\{v_{i}\right\}\right)$ in the obvious way. The sign factor in front of $T^{\prime \prime}$ is obtained by keeping track of the reordering of non-root edges of $T^{\prime}$ and $T$.

Let us now consider the case when $v_{i}$ has $q \geq 1$ incoming edges. Since the tree $T^{\prime}$ is planar these $q$ incoming edges are totally ordered. So we denote them by $e_{1}, e_{2}, \ldots, e_{q}$ keeping in mind that

$$
e_{1}<e_{2}<\cdots<e_{q} \text {. }
$$

The desired vector $T^{\prime} \circ_{i} T \in \mathrm{Tw} \mathrm{BT}(n+k-1)$ is represented by the sum

$$
T^{\prime} \circ_{i} T=\sum_{\alpha}(-1)^{f(\alpha)} T_{\alpha}
$$


where $T_{\alpha}$ is obtained from $T^{\prime}$ and $T$ following these steps:

- first, we erase the vertex $v_{i} \in V_{\mathrm{lab}}\left(T^{\prime}\right)$ and glue the tree $T$ via identifying the root edge $T$ with the edge originating from $v_{i}$,

- second, we attach the edges $e_{1}, e_{2}, \ldots, e_{q}$ to vertices in the set $V_{\text {lab }}(T) \sqcup V_{\nu}(T)$

- finally, we relabel elements of the set $V_{\text {lab }}\left(T^{\prime \prime}\right)=V_{\text {lab }}(T) \sqcup\left(V_{\text {lab }}\left(T^{\prime}\right) \backslash\left\{v_{i}\right\}\right)$ in the obvious way.

Ways of connecting the edges $e_{1}, e_{2}, \ldots, e_{q}$ to vertices in the set $V_{\text {lab }}(T) \sqcup V_{\nu}(T)$ should obey the obvious analog of Condition 7.1 .

Condition 8.1. The restriction of the total order on the set $E\left(T_{\alpha}\right)$ of $T_{\alpha}$ to the subset $\left\{e_{1}, e_{2}, \ldots, e_{q}\right\}$ should coincide with the order 8.8 .

The summation in (8.9) goes over all ways $\alpha$ of connecting the edges $e_{1}, e_{2}, \ldots, e_{q}$ to vertices in the set $V_{\text {lab }}(T) \sqcup V_{\nu}(T)$ satisfying Condition 8.1 . The sign factor $(-1)^{f(\alpha)}$ is define in the same way as for elementary insertions in BT.

Example 8.2. Let $T_{\circ \circ}$ (resp. $T_{\cup}$ ) be the brace tree in $\mathcal{T}^{\mathrm{tw}}(2)$ depicted on figure 7.3 (resp. figure 8.1). Then the vector $T \circ_{1} T \cup \in \mathrm{Tw} \mathrm{BT}(3)$ equals to the sum shown on figure 8.5 .

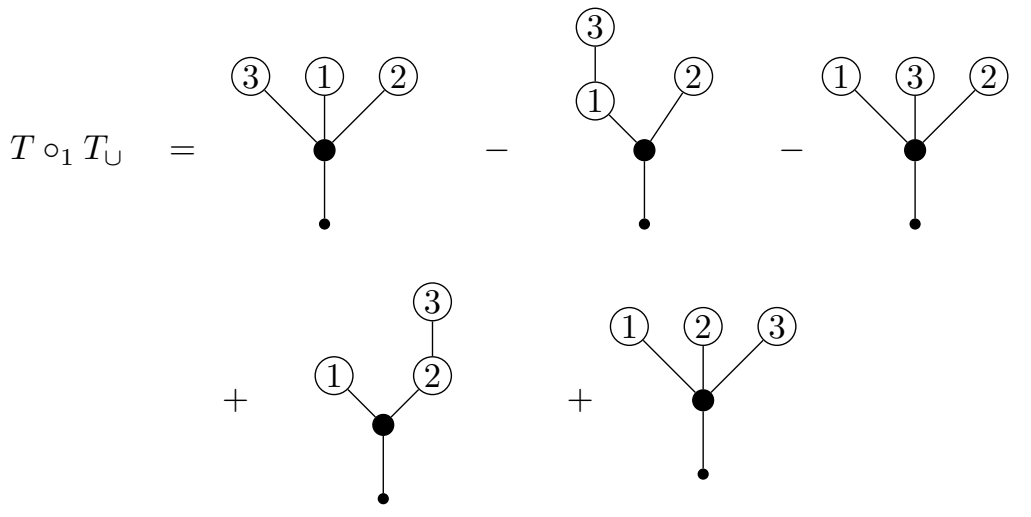

FIG. 8.5. The elementary insertion $T \circ_{1} T_{\cup}$

Remark 8.3. Let us recall that $\mathrm{BT}(0)=\mathbf{0}$. Hence, due to Remark 3.7, the graded vector space of the $d g$ Lie algebra

$$
\mathcal{L}_{\mathrm{BT}}=\operatorname{Conv}\left(\Lambda^{2} \text { coCom, BT }\right)
$$

is canonically identified with $\mathbf{s}^{-2} \mathrm{Tw} \mathrm{BT}(0)$ Thus, we can use brace trees in $\mathcal{T}^{\mathrm{tw}}(0)$ to represent vectors in the dg Lie algebra (8.10).

However, the degree formula should be adjusted as follows: a brace tree $T \in \mathcal{T}^{\mathrm{tw}}(0)$ representing a vector in $\mathcal{L}_{\mathrm{BT}}$ carries the degree

$$
|T|=2\left|V_{\nu}(T)\right|-|E(T)|-1 .
$$

For example, the vector T.• depicted on figure 8.6 is the Maurer-Cartan element in $\mathcal{L}_{\mathrm{BT}}$ corresponding to the map 8.1). This vector carries degree 1 . 
FIG. 8.6. The brace tree $T_{\bullet \bullet} \in \mathcal{T}^{\mathrm{tw}}(0)$

8.1. The differential on Tw BT. Following the general procedure, the differential $\partial(T)$ of a brace tree $T \in \mathrm{Tw} \mathrm{BT}(n)$ is given by the formula

$$
\partial(T)=T_{\bullet \bullet} \cdot T+\kappa\left(T_{\bullet \bullet}\right) \circ_{1} T-(-1)^{|T|} \sum_{i=1}^{n} T \circ_{i} \kappa(T \bullet \bullet) .
$$

Here $T_{\bullet \bullet} \cdot$ denotes the auxiliary action 3.13 of the Lie algebra $\mathcal{L}_{\mathrm{BT}}$ on the operad Tw BT and

$$
\kappa\left(T_{\bullet \bullet}\right)=T_{\bullet 1}+T_{1 \bullet}
$$

where $T_{\bullet 1}$ and $T_{1}$ are the brace trees depicted on figures 8.3 and 8.4 , respectively. Unfolding the definition of the auxiliary action $(3.13)$ and using $(8.13)$ we get

$$
\begin{aligned}
\partial(T)= & -(-1)^{|T|} \sum_{v \in V_{\nu}(T)} T_{v} \circ_{n+1} T_{\bullet \bullet}+T_{1 \bullet} \circ_{1} T+T_{\bullet 1} \circ_{1} T \\
& -(-1)^{|T|} \sum_{i=1}^{n} T \circ_{i} T_{1} \bullet(-1)^{|T|} \sum_{i=1}^{n} T \circ_{i} T \bullet 1 .
\end{aligned}
$$

where $T_{v}$ is a brace tree in $\mathcal{T}^{\mathrm{tw}}(n+1)$ which is obtained from $T$ by replacing the neutral vertex $v$ by a "white" vertex with label $n+1$. In other words, $T_{\bullet \bullet} \cdot T$ is the sum over insertions of the brace tree $T_{\bullet \bullet}$ (see fig. 8.6 into neutral vertices of $T$ with appropriate signs. The expression $T_{1} \bullet{ }_{1} T$ is the sum of brace trees which are obtained from $T$ by attaching the single edge with a neutral vertex on one end to all non-root vertices of $T$. The brace tree $T_{\bullet 1} \circ_{1} T$ is obtained from the tree $T$ by dividing the root edge into two parts and inserting a neutral vertex in the middle. The expression $T \circ_{i} T_{1} \bullet$ (resp. the expression $\left.T \circ_{i} T \bullet 1\right)$ is obtained from $T$ by inserting $T_{1} \bullet\left(\operatorname{resp} . T_{\bullet 1}\right)$ into the vertex of $T$ with label $i$.

Example 8.4. Let $T_{\circ \circ}, T_{\cup}, T_{\cup \text { opp }}, T_{\bullet 1}$ be the brace trees depicted on figures 7.3, 8.1, 8.2. and 8.3. Direct computations show that $T_{\cup}$ and $T_{\cup \text { opp }}$ are $\partial$-closed and $T_{\circ \circ}$ is not $\partial$-closed. Instead, we have

$$
\partial\left(T_{\circ \circ}\right)=T_{\cup o p p}-T_{\cup}
$$

In other words, $T_{\cup}$ is cohomologous to $T_{\cup \text { opp }}$.

For the brace tree $T_{\bullet 1}$ we have

$$
\partial\left(T_{\bullet 1}\right)=T_{\bullet \bullet 1}
$$

where $T_{\bullet \bullet 1}$ is the brace tree depicted on figure 8.7 .

The operad Tw BT acts naturally on the Hochschild cochain complex $C^{\bullet}(A)$ of any (flat) $A_{\infty}$-algebra $A$. This action is described in Appendix B. 


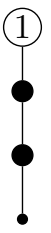

FIG. 8.7. The brace tree $T_{\bullet \bullet 1} \in \mathcal{T}^{\mathrm{tw}}(1)$

\section{The braces operad Br À la Kontsevich-Soibelman}

In this section we define a suboperad $\mathrm{Br}$ of $\mathrm{Tw} \mathrm{BT}$. The operad $\mathrm{Br}$ coincides with what $\mathrm{M}$. Kontsevich and Y. Soibelman called the "minimal operad" in 21. By slightly abusing notation we will call $\mathrm{Br}$ the operad of braces and we will refer to algebras over $\mathrm{Br}$ as brace algebras. At the end of this section we will show that the embedding $\mathrm{Br} \hookrightarrow \mathrm{Tw} \mathrm{BT}$ is a quasi-isomorphism of operads.

We will say that a brace tree $T \in \mathcal{T}^{\mathrm{tw}}(n)$ is admissible if $T$ satisfies the following condition:

Condition 9.1. Every neutral vertex of $T$ has at least 2 incoming edges.

Thus figures 7.3 , 8.1, and 8.2 show examples of admissible brace trees while figures 8.3 , 8.4 , 8.6 , show examples of inadmissible brace trees.

We define $\operatorname{Br}(n)$ as the subspace of $\operatorname{Tw} \operatorname{BT}(n)$ which is spanned by admissible brace trees. It is easy to see that, in $\mathcal{T}^{\mathrm{tw}}(0)$ there are no admissible brace trees. Hence,

$$
\operatorname{Br}(0)=\mathbf{0} .
$$

Furthermore, in $\mathcal{T}^{\mathrm{tw}}(1)$, there is exactly one admissible brace tree. This brace tree is depicted on figure 7.2 Thus

$$
\operatorname{Br}(1)=\mathbb{K}
$$

We claim that

Proposition 9.2. The sub-collection $\{\operatorname{Br}(n)\}_{n \geq 0}$ is a suboperad of $\mathrm{Tw} \mathrm{BT}$.

Proof. It is obvious that for admissible brace trees $T^{\prime} \in \mathcal{T}^{\text {tw }}(k)$ and $T \in \mathcal{T}^{\text {tw }}(n)$

$$
T^{\prime} \circ_{i} T, \quad 1 \leq i \leq k
$$

are linear combinations of admissible brace trees. Thus it remains to prove that for every brace tree $\partial T$ is a linear combination of admissible brace trees. Concretely, we have to show that in $\partial T$ there occur no brace trees with 1- or 2-valent neutral vertices.

Let $e(T)$ be the number of all edges of $T$. The vertex splitting operations produce $2 e(T)$ terms with a 2 -valent neutral vertex, 2 for each of the $e(T)$ edges. Concretely,

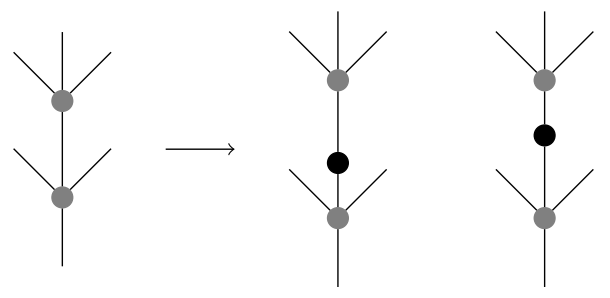


Here the left term comes from splitting of the lower gray vertex and the right term comes from splitting of the upper gray vertex. The upper gray vertex can be either labeled or neutral, in particular, it may have valency 1 . The lower gray vertex may be either a labeled vertex, or a neutral vertex, or the root vertex. (Of course, in the latter case the lower vertex has valency 1.) The remainder of the brace tree is omitted. Comparing the signs factors, we see that these two terms cancel each other, so the differential does not produce valence 2 neutral vertices.

Next consider valence 1 neutral vertices. Again, the corresponding brace trees come in pairs:

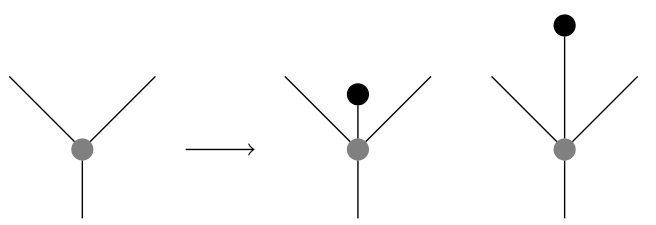

Here the left term comes from splitting of the shown gray (i.e., white or black) vertex and the right term comes from the linear combination $\kappa\left(T_{\bullet \bullet}\right) \circ_{1} T$ in 8.12 . Again, checking the signs, we see that these two terms cancel each other and no valence 1 neutral vertex is produced.

9.1. A simpler description of the differential on $\operatorname{Br}$. Let $T$ be an admissible brace tree in $\mathcal{T}^{\text {tw }}(n), v$ be a vertex in $V_{\text {lab }}(T)$ carrying label $i$, and $\kappa\left(T_{\bullet \bullet}\right)$ be the sum $T_{\bullet 1}+T_{1}$ of inadmissible brace trees depicted on figures 8.3 and 8.4 . We denote by $\partial_{v}(T)$ a vector in $\operatorname{Br}(n)$ which is obtained from the sum

$$
T \circ_{i} \kappa\left(T_{\bullet \bullet}\right)
$$

by omitting all non-admissible brace trees. Let $v$ be a neutral vertex of $T$. To define $\partial_{v}(T)$ we change the color of vertex $v$ to white and assign to $v$ label $n+1$. We denote this new brace tree by $T_{v}$ and obtain $\partial_{v}(T)$ from the sum

$$
T_{v} \circ_{n+1} T_{\bullet \bullet}
$$

via omitting all non-admissible brace trees.

Finally, we declare that the differential $\partial$ on $\operatorname{Br}$ is the vector $\partial(T) \in \operatorname{Br}(n)$ is represented by the sum

$$
\partial(T)=\sum_{v \in V_{\mathrm{lab}}(T) \sqcup V_{\nu}(T)} \pm \partial_{v}(T),
$$

where the sign factors are determined in the same way as in formula 8.12 .

9.2. The inclusion $\mathrm{Br} \hookrightarrow$ Tw BT is a quasi-isomorphism. The goal of this section is to prove the following theorem

Theorem 9.3. The operad map

$$
\mathrm{Br} \hookrightarrow \mathrm{Tw} \text { BT }
$$

is a quasi-isomorphism.

Proof. Let $T$ be a brace tree in $\mathcal{T}^{\mathrm{tw}}(n)$ and let $\nu_{\leq 2}(T)$ be the total number of neutral vertices of valence $\leq 2$. For example, a brace tree $T$ is admissible if and only if

$$
\nu_{\leq 2}(T)=0 .
$$


We observe that for every brace tree $T \in \mathcal{T}^{\mathrm{tw}}(n)$ the linear combination

$$
\partial(T)
$$

involves brace trees $T_{i}$ with $\nu_{\leq 2}\left(T_{i}\right)=\nu_{\leq 2}(T)$ or $\nu_{\leq 2}\left(T_{i}\right)=\nu_{\leq 2}(T)+1$.

This observation allows us to introduce on Tw BT the following increasing filtration

$$
\cdots \subset \mathcal{F}^{m-1} \mathrm{Tw} \text { BT } \subset \mathcal{F}^{m} \mathrm{Tw} \text { BT } \subset \mathcal{F}^{m+1} \mathrm{Tw} \text { BT } \subset \ldots,
$$

where $\mathcal{F}^{m}$ Tw BT $(n)$ consists of linear combinations of brace trees $T \in \mathcal{T}^{\mathrm{tw}}(n)$ satisfying the condition:

$$
\nu_{\leq 2}(T)-\operatorname{deg}(T) \leq m
$$

The restriction of (9.4) on Br gives us the "silly" filtration

$$
\mathcal{F}^{m} \operatorname{Br}(n)^{k}= \begin{cases}\operatorname{Br}(n)^{k} \quad \text { if } k \geq-m \\ 0 \quad \text { otherwise }\end{cases}
$$

with the associated graded complex carrying the zero differential.

To describe the associated graded complex for Tw BT, we introduce the notion of a core for brace trees in $\mathcal{T}^{\mathrm{tw}}(n)$. Namely, for a brace tree $\Gamma \in \mathcal{T}^{\mathrm{tw}}(n)$, its core $\Gamma^{\prime}$ obtained by deleting all neutral bivalent vertices and connecting their incident edges. Here is an example:

a brace tree

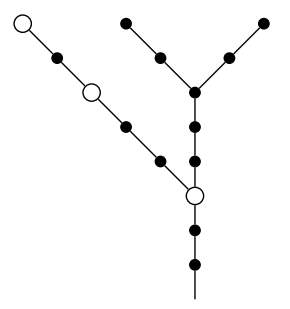

its core

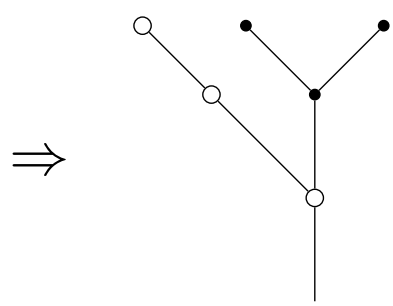

It is easy to see that the associated graded complex

$$
\operatorname{Gr} \operatorname{Tw} \operatorname{BT}(n)
$$

splits into the direct sum over all possible cores with $n$ labeled vertices

$$
\bigoplus_{\operatorname{cores} \Gamma^{\prime}} \mathrm{Tw}_{\mathrm{B}} \Gamma_{\Gamma^{\prime}}
$$

where $\mathrm{Tw}_{\mathrm{B}} \mathrm{BT}_{\Gamma^{\prime}}$ is spanned by brace trees with the given core $\Gamma^{\prime}$.

Furthermore, for each core $\Gamma^{\prime}$ the subcomplex $\mathrm{Tw} B T_{\Gamma^{\prime}}$ is isomorphic to the tensor product

$$
\mathrm{Tw} \mathrm{BT}_{\Gamma^{\prime}} \cong \bigotimes_{\text {edges } e \text { of } \Gamma^{\prime}} V_{e}
$$

where

$$
V_{e}= \begin{cases}\mathbb{K} \stackrel{i d}{\rightarrow} \mathbb{K} \stackrel{0}{\rightarrow} \mathbb{K} \stackrel{i d}{\rightarrow} \mathbb{K} \cdots & \text { if } e \text { connects to a univalent neutral vertex } \\ \mathbb{K} \stackrel{0}{\rightarrow} \mathbb{K} \stackrel{i d}{\rightarrow} \mathbb{K} \stackrel{0}{\rightarrow} \mathbb{K} \cdots & \text { otherwise }\end{cases}
$$


The various copies of $\mathbb{K}$ correspond to strings of bivalent neutral vertices of various lengths:

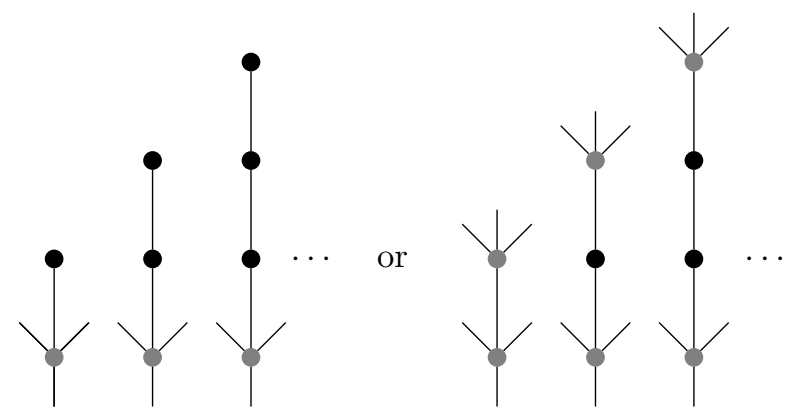

Here, a gray vertex is either a labeled vertex or a neutral vertex with $\geq 2$ incoming edges. Any lower gray vertex may also be the root vertex. The rest of the brace tree is omitted, and just indicated by some stubs at the gray vertices. Clearly the cohomology of $V_{e}$ is trivial if $e$ connects to a univalent neutral vertex and $\mathbb{K}$ otherwise. The representative in the latter case is a single edge (i.e., a string of zero neutral vertices).

Therefore the cochain complex $\mathrm{Tw}_{\mathrm{BT}} \Gamma_{\Gamma^{\prime}}$ is acyclic if $\Gamma^{\prime}$ contains at least one univalent neutral vertex and

$$
H^{\bullet}\left(\mathrm{Tw} \mathrm{BT}_{\Gamma^{\prime}}\right) \cong \mathbb{K}\left\langle\left\{\Gamma^{\prime}\right\}\right\rangle
$$

if the core $\Gamma^{\prime}$ does not have univalent neutral vertices. Hence the embedding

$$
\operatorname{Br}(n) \hookrightarrow \operatorname{Tw} \operatorname{BT}(n)
$$

induces a quasi-isomorphism on the level of associated graded complexes.

Combining this observation with the fact that the filtrations (9.4) and (9.6) are cocomplete and locally bounded, we deduce Theorem 9.3 from [8, Lemma A.3].

9.3. The dg operad $\mathrm{Br}$ is a homotopy fixed point of $\mathrm{Tw}$. Various solutions of the Deligne conjecture on Hochschild cochain complex [1, 2], 29, 26, 27, 21], 31, 32] imply that the dg operad $\mathrm{Br}$ is quasiisomorphic to the dg operad

$$
C_{-\bullet}\left(E_{2}, \mathbb{K}\right)
$$

of singular chains for the little disc operad $E_{2}$.

Combining this statement with the formality [20, 30] for the $\operatorname{dg}$ operad $C_{-} \bullet\left(E_{2}, \mathbb{K}\right)$, we conclude that the dg operad $\mathrm{Br}$ (as well as Tw BT) is quasi-isomorphic to the operad Ger.

Thus Theorem 5.1 and Corollary 5.12 imply that

Corollary 9.4. The dg operads $\mathrm{Br}$ and $\mathrm{Tw} \mathrm{BT}$ are homotopy fixed points for $\mathrm{Tw}$.

9.4. $\mathrm{Br}$ is a $\mathrm{Tw}$-coalgebra. Consider the composition

$$
\mathrm{Br} \rightarrow \mathrm{Tw} \mathrm{BT} \rightarrow \mathrm{Tw} \mathrm{Tw} \mathrm{BT} .
$$

We claim that the image actually lands in $\mathrm{Tw} B r \subset \mathrm{Tw} T w \mathrm{BT}$. Indeed, neither the first nor the second map creates new neutral vertices, and hence no neutral vertices of valence 1 or 2 in particular. Hence we have a map

$$
c: \mathrm{Br} \rightarrow \mathrm{Tw} \mathrm{Br} \text {. }
$$

It follows almost immediately from the Tw colagebra axioms for Tw BT that this map endows $\mathrm{Br}$ with the structure of a Tw-coalgebra. Summarizing, we arrive at the following result. 
Lemma 9.5. $\mathrm{Br}$ is a sub $\mathrm{Tw}$-coalgebra of $\mathrm{Tw} \mathrm{BT}$.

10. Every solution of Deligne's conjecture is homotopic to a one that is Compatible with TWISTING

We are now ready to prove Theorem 1.1 which roughly states that every solution of Deligne's conjecture is homotopic to a one that is compatible with twisting.

We will deduce this result from the following more general statement:

Theorem 10.1. Let $\Lambda \mathrm{Lie}_{\infty} \rightarrow \mathcal{O}, \Lambda \mathrm{Lie}_{\infty} \rightarrow \mathcal{O}^{\prime}$ be two objects of the under-category $\Lambda \mathrm{Lie}_{\infty} \downarrow$ Operads and let $\mathcal{O} \stackrel{F}{\rightarrow} \mathrm{Tw} \mathcal{O}^{\prime}$ be a morphism of $d g$ operads compatible with the maps from $\Lambda \mathrm{Lie}_{\infty}$. If $(\mathcal{O}, c)$ is a $\mathrm{Tw}$-coalgebra and the $d g$ operads $\mathcal{O}, \mathrm{Tw}_{\mathrm{O}} \mathcal{O}^{\prime}$ are homotopy fixed points for $\mathrm{Tw}$, then the morphism

$$
F^{\prime}:=\left(\operatorname{Tw}\left(\eta_{\mathcal{O}^{\prime}} \circ F\right)\right) \circ c: \mathcal{O} \rightarrow \operatorname{Tw} \mathcal{O}^{\prime}
$$

is homotopy equivalent to $F$. Furthermore, $F^{\prime}$ is a morphism of $\mathrm{Tw}$-coalgebras.

This theorem can be restated as follows: if $\mathcal{O}$ and $\mathrm{Tw} \mathcal{O}^{\prime}$ are homotopy fixed points for $\mathrm{Tw}$ (with $\mathcal{O}$ being a Tw-coalgebra) then each homotopy class of maps of $\operatorname{dg}$ operads $\mathcal{O} \rightarrow \mathrm{Tw} \mathcal{O}^{\prime}$ has at least one representative which respects the $\mathrm{Tw}$-coalgebra structures on $\mathcal{O}$ and $\mathrm{Tw} \mathcal{O}^{\prime}$. Furthermore, such a representative may be written down explicitly.

Remark 10.2. The homotopy category of dg operads can be constructed using the standard technics of closed model categories. For more details we refer the reader to [16], 17], and [28, Part II, Appendix A.4]. In the proof below we use merely the existence of the homotopy category of $d g$ operads.

Proof. Abbreviate $f:=\eta_{\mathcal{O}^{\prime}} \circ F$ and consider the following commutative diagram:

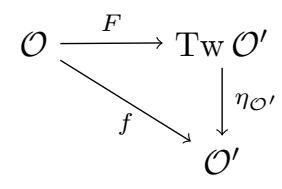

Applying the functor $\mathrm{Tw}$ to 10.2 we obtain the commutative diagram:

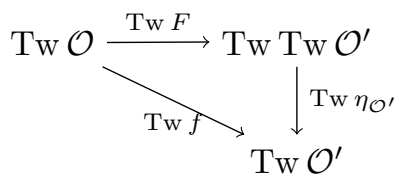

Next consider the following diagram:

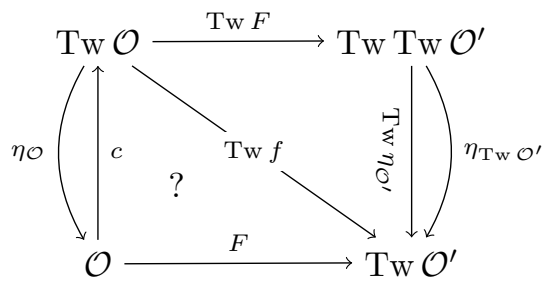

Our goal is to show that the triangle marked with "?" is homotopy commutative. We will do this by showing that all the other cells (including the big cell around the diagram) homotopy commute. We saw above that 
the upper right triangle commutes. Furthermore the big outer square commutes because $\eta$ is a natural transformation. The small cell on the left commutes since $\eta_{\mathcal{O}} \circ c=i d_{\mathcal{O}}$ by the definition of a Tw coalgebra. Finally consider the small cell on the right. Note that, by the defining property of the counit, the following diagram commutes:

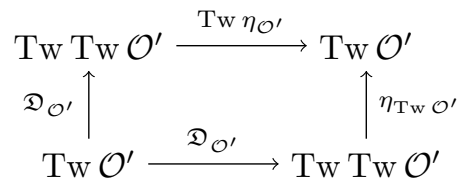

All arrows are quasi-isomorphisms since $\mathrm{Tw} \mathcal{O}^{\prime}$ is a homotopy fixed point of $\mathrm{Tw}$. Hence the morphisms $\mathrm{Tw} \eta_{\mathcal{O}^{\prime}}$ and $\eta_{\mathrm{Tw}} \mathcal{O}^{\prime}$ are homotopic. This shows that the triangle? in the previous diagram is homotopy commutative.

It remains to prove that $F^{\prime} 10.1$ is a morphism of Tw-coalgebras.

For this purpose we consider the following diagram

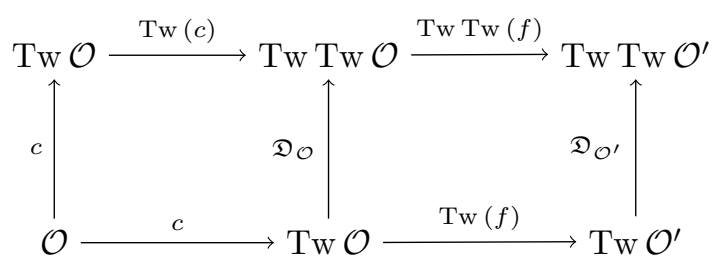

The left square of diagram 10.5 commutes since $c: \mathcal{O} \rightarrow$ Tw $\mathcal{O}$ is the comultiplication map for the Tw-coalgebra $\mathcal{O}$. The right square of diagram 10.5 commutes since $\mathfrak{D}$ is a natural transformation from Tw to Tw Tw. Thus the ambient rectangle forms a commutative diagram.

On the other hand, the composition $\mathrm{Tw}(f) \circ c$ of the lower horizontal arrows is $F^{\prime}$ and the composition Tw Tw $(f) \circ \mathrm{Tw}(c)$ of upper horizontal arrows is $\mathrm{Tw}\left(F^{\prime}\right)$. Hence $F^{\prime}$ is indeed a morphism of Tw -coalgebras.

Theorem 10.1 is proved.

In order to prove Theorem 1.1 we need a technical property of operad maps from Ger $\infty$ to BT.

So let $f:$ Ger $_{\infty} \rightarrow$ BT be a map of dg operads and let

$$
\alpha \in \operatorname{Conv}\left(\operatorname{Ger}^{\vee}, \mathrm{BT}\right) \cong \prod_{n \geq 2}\left(\mathrm{BT}(n) \otimes \Lambda^{-2} \operatorname{Ger}(n)\right)^{S_{n}}
$$

be the Maurer-Cartan element which corresponds to $f$.

Using the fact that every vector in $\mathrm{BT}(n)$ carries degree $1-n$, we deduce that $\alpha$ has the form

$$
\alpha=\sum_{n \geq 2, i} \sum_{\sigma \in S_{n}} \sigma\left(X_{n, i} \otimes v_{n, i}^{1} v_{n, i}^{2}\right)
$$

where $X_{n, i} \in \mathrm{BT}(n)$ and

$$
v_{n, i}^{1} v_{n, i}^{2} \in \mathbf{s}^{2} S^{2}\left(\Lambda^{-1} \operatorname{Lie}\left(b_{1}, \ldots, b_{n}\right)\right) \cap \Lambda^{-2} \operatorname{Ger}(n)
$$

We claim that

Proposition 10.3. If $f: \mathrm{Ger}_{\infty} \rightarrow \mathrm{BT}$ is an operad map satisfying the condition

$$
f\left(\mathbf{s} 1_{2}^{\mathfrak{c}}\right)=T_{\circ \circ}+\sigma_{12}\left(T_{\circ \circ}\right)
$$


with $T_{\circ}$ being the brace tree shown on figure 7.3, then the Maurer-Cartan element $\alpha$ does not involve monomials of the form

$$
T \otimes b_{1} v\left(b_{2}, \ldots b_{n}\right), \quad n \geq 3,
$$

where $v$ is a $\Lambda^{-1}$ Lie-monomial and $T$ is a brace tree with vertex (1) having valency 1 or 2 .

We will postpone the technical proof of this proposition to Section 10.1 and complete the proof of Theorem 1.1 .

According to Section $4.3, \mathrm{Ger}_{\infty}$ is a Tw-coalgebra. Furthermore, by Corollaries 5.13 and 9.4 the dg operads $\mathrm{Ger}_{\infty}$ and Tw BT are homotopy fixed points for $\mathrm{Tw}$. Thus, given an operad quasi-isomorphism $F: \mathrm{Ger}_{\infty} \rightarrow \mathrm{Br}$ we can apply Theorem 10.1 to $\mathcal{O}=\mathrm{Ger}_{\infty}, \mathcal{O}^{\prime}=\mathrm{BT}$ and the composition

$$
\widetilde{F}: \operatorname{Ger}_{\infty} \stackrel{F}{\longrightarrow} \operatorname{Br} \hookrightarrow \operatorname{Tw} B T .
$$

Since $\widetilde{F}$ is a quasi-isomorphism from $\mathrm{Ger}_{\infty}$ to $\mathrm{Tw} \mathrm{BT}$, the composition

$$
f=\eta_{\mathrm{BT}} \circ \widetilde{F}: \operatorname{Ger}_{\infty} \rightarrow \mathrm{BT}
$$

satisfies condition (10.8). Hence, applying Proposition 10.3 we conclude that for every basis vector $v \in$ $\Lambda^{-1} \operatorname{Lie}(n-1)$ the linear combination

$$
\eta_{\mathrm{BT}} \circ \widetilde{F}\left(\mathbf{s}\left(b_{1} v\left(b_{2}, \ldots b_{n}\right)\right)^{*}\right) \in \mathrm{BT}(n)
$$

does not involve brace trees with vertex (1) having valency $\leq 2$.

This observation implies that the composition

$$
\widetilde{F}^{\prime}=\operatorname{Tw}(f) \circ c: \operatorname{Ger}_{\infty} \rightarrow \mathrm{Tw} \mathrm{BT}
$$

factors through the sub-operad $\mathrm{Br}$.

Since $\widetilde{F}^{\prime}$ is homotopy equivalent to $\widetilde{F}$, and $\widetilde{F}^{\prime}$ is compatible with the Tw-coalgebra structures, Theorem 1.1 follows.

10.1. The proof of Proposition 10.3. The proof of Theorem 1.1 is based on technical Proposition 10.3 Here we give a proof of this proposition.

In the proof of this proposition, it is more convenient to use a different but equivalent description of the pre-Lie algebra $\operatorname{Conv}(Q, P)$ which makes sense if each $Q(n)$ is finite dimensional. This description is given in Appendix C.

Condition 10.8 implies that $\alpha$ has the form

$$
\alpha=\alpha_{0}+\sum_{n \geq 3, i} \sum_{\sigma \in S_{n}} \sigma\left(X_{n, i} \otimes v_{n, i}^{1} v_{n, i}^{2}\right),
$$

where, as above, $X_{n, i} \in \mathrm{BT}(n)$,

$$
v_{n, i}^{1} v_{n, i}^{2} \in \mathbf{s}^{2} S^{2}\left(\Lambda^{-1} \operatorname{Lie}\left(b_{1}, \ldots, b_{n}\right)\right) \cap \Lambda^{-2} \operatorname{Ger}(n),
$$

and $\alpha_{0}$ is the degree 1 vector

$$
\alpha_{0}:=T_{\circ \circ} \otimes b_{1} b_{2}+\sigma_{12}\left(T_{\circ \circ}\right) \otimes b_{1} b_{2} .
$$


Recall that, due to Proposition C.2 from Appendix C, the map Av (C.3) gives us an isomorphism to the pre-Lie algebra

$$
\operatorname{Conv}\left(\operatorname{Ger}^{\vee}, \mathrm{BT}\right) \cong \prod_{n \geq 2}\left(\mathrm{BT}(n) \otimes \Lambda^{-2} \operatorname{Ger}(n)\right)^{S_{n}}
$$

from the pre-Lie algebra

$$
\prod_{n \geq 2}\left(\mathrm{BT}(n) \otimes \Lambda^{-2} \operatorname{Ger}(n)\right)_{S_{n}}
$$

with the pre-Lie product $\bullet^{\prime}$ given by equation (C.6). So, in our consideration, we may replace $\operatorname{Conv}\left(\operatorname{Ger}^{\vee}, B T\right)$ by the pre-Lie algebra 10.11).

Let us denote by $\mathcal{T}^{\prime}(n)$ the set of all brace trees for which the labeling agrees with the natural order on the set of vertices. For example, the brace tree $T_{\circ \circ}$ on figure 7.3 belongs to $\mathcal{T}^{\prime}(2)$ and $\sigma_{12}\left(T_{\circ \circ}\right)$ does not belong to $\mathcal{T}^{\prime}(2)$.

Since BT $(n)$ is a free $S_{n}$-module generated elements of $\mathcal{T}^{\prime}(n)$ we conclude that

$$
\left(\mathrm{BT}(n) \otimes \Lambda^{-2} \operatorname{Ger}(n)\right)_{S_{n}} \cong \mathbb{K}\left\langle\mathcal{T}^{\prime}(n)\right\rangle \otimes \Lambda^{-2} \operatorname{Ger}(n) .
$$

Hence the vector space 10.12 has the following natural basis

$$
\left\{T \otimes w_{n, i}\right\}_{T \in \mathcal{T}^{\prime}(n), i \in I_{n}},
$$

where $w_{n, i}$ are vectors of the basis 4.17 for $\Lambda^{-2} \operatorname{Ger}(n)$.

We also observe that, the $\Lambda^{-1}$ Lie-monomials

$$
\left.\left\{v_{\tau}=\left\{b_{\tau(1)},\left\{b_{\tau(2)}, \ldots b_{\tau(n-2)}, b_{n-1}\right\}\right\} . . .\right\}\right\}_{\tau \in S_{n-2}}
$$

form a basis in the vector space $\Lambda^{-1} \operatorname{Lie}(n-1)$ and

$$
\left\{b_{i} v_{\tau}\left(b_{1}, \ldots, \widehat{b}_{i}, \ldots, b_{n}\right)\right\}_{1 \leq i \leq n ; \tau \in S_{n-2}}
$$

is a subset of the basis 4.17) for $\Lambda^{-2} \operatorname{Ger}(n)$.

Our goal is to show that the decomposition of the Maurer-Cartan element $\mathrm{Av}^{-1}(\alpha)$ with respect to the basis 10.13 does not involve vectors of the form

$$
T \otimes b_{i} v_{\tau}\left(b_{1}, \ldots, \widehat{b_{i}}, \ldots, b_{n}\right),
$$

where $T$ is a brace tree in $\mathcal{T}^{\prime}(n)$ with vertex (i) being either univalent or bivalent. So the "forbidden" brace trees locally have one of the three forms shown schematically in figures 10.1, 10.2, and 10.3. Gray vertices on these figures are some other labeled vertices.

Let us introduce an operation $V_{i}^{\circ \circ}$ whose input is a brace tree in $\mathcal{T}^{\prime}(n)$ with vertex (i) being either univalent or bivalent. The construction of the output $V_{i}^{\circ-\circ}(T)$ depends on whether vertex (i) is univalent or bivalent.

If vertex (i) of $T$ is univalent then $V_{i}^{\circ-\circ}(T)$ is a brace tree in $\mathcal{T}^{\prime}(n+1)$ which is obtained from $T$ via

- shifting labels of $T$ which are $>i$ up by 1 ,

- creating a vertex with label $i+1$, and

- attaching the vertex with label $i+1$ to the vertex with label $i$ by a single edge.

If vertex (i) of $T$ is bivalent then $V_{i}^{\circ-\circ}(T)$ is a brace tree in $\mathcal{T}^{\prime}(n+1)$ which is obtained from $T$ via

- shifting labels of $T$ which are $>i$ up by 1 , 


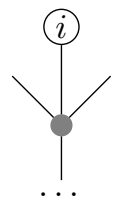

FIG. $\quad 10.1$.

Vertex $i$ is

univalent

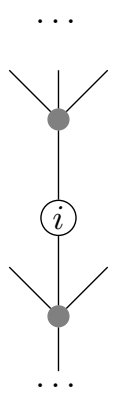

FIG. 10.2. Bivalent vertex $i$ is not adjacent to the root

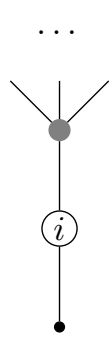

FIG. 10.3. Bivalent vertex $i$ is adjacent to the root

- creating a vertex with label $i+1$,

- detaching the edge terminating at the vertex with label $i$ and attaching it to the vertex with label $i+1$

- and finally, attaching the vertex with label $i+1$ to the label $i$ by a single edge.

In pictures, $V_{i}^{\circ-\circ}(T)$ locally looks as follows:
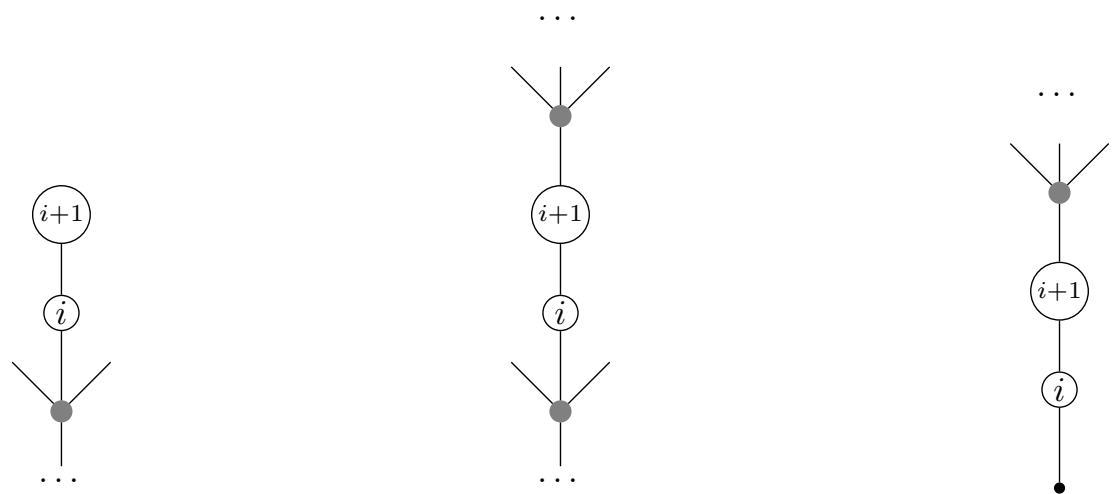

Unfolding the definition, it is not hard to see that for every vector of the form 10.16

$$
\begin{aligned}
\alpha_{0} \bullet^{\prime} T & \otimes b_{i} v_{\tau}\left(b_{1}, \ldots, \widehat{b_{i}}, \ldots, b_{n}\right)+T \otimes b_{i} v_{\tau}\left(b_{1}, \ldots, \widehat{b_{i}}, \ldots, b_{n}\right) \bullet^{\prime} \alpha_{0}= \\
& \pm V_{i}^{\circ-\circ}(T) \otimes b_{i} b_{i+1} v_{\tau}\left(b_{1}, \ldots, b_{i-1}, b_{i+2}, \ldots, b_{n}\right)+\ldots,
\end{aligned}
$$

where ... denote the linear combination of the remaining vectors in the basis for (10.12).

Remark 10.4. Note that graphically the operation $\bullet^{\prime} \alpha_{0}$ is the summation over splittings of a labeled vertex into two labeled vertices, while the operation $\alpha_{0} \bullet^{\prime}$ may graphically be interpreted as attaching a new labeled vertex in all possible ways "from the top" and "from the bottom" of the tree. Hence the first term on the right hand side of (10.17) is produced three times on the left hand side of (10.17). If $T$ is of the form shown on figure 10.1 or of the form shown on figure 10.3 then the first contribution comes from the operation $\alpha_{0} \bullet^{\prime}$, the second one comes from splitting vertex $i$ and the third one comes from splitting the labeled vertex adjacent to vertex $i$. If $T$ is of the form shown on figure 10.2 then all these three contributions come from the operation $\bullet^{\prime} \alpha_{0}$ : the first one comes from the splitting vertex $i$ and the other two contributions come from 
splitting the labeled vertices adjacent to vertex $i$. We claim that in all three cases the signs let two of the three contributions cancel.

It is clear that, if $v v^{\prime}$ (resp. $w$ ) is a vector in $\Lambda^{-2} \operatorname{Ger}(n)$ (resp. in $\Lambda^{-2} \operatorname{Ger}(m)$ ) with the $\Lambda^{-1}$ Lie-words $v, v^{\prime}$ having length $\geq 2$, then the pre-Lie products

$$
\left(T_{1} \otimes v v^{\prime}\right) \bullet^{\prime}\left(T_{2} \otimes w\right)
$$

and

$$
\left(T_{2} \otimes w\right) \bullet\left(T_{1} \otimes v v^{\prime}\right)
$$

do not involve tensor factors of the form $b_{i} b_{i+1} v_{\tau}\left(b_{1}, \ldots, b_{i-1}, b_{i+2}, \ldots, b_{n}\right)$.

It is also clear that, if the vertex with label $j_{1}$ (resp. label $j_{2}$ ) of a brace tree $T_{1} \in \mathcal{T}^{\prime}(n)$ (resp. $T_{2} \in$ $\left.\mathcal{T}^{\prime}(m)\right)$ has valency $\geq 3$, then for every pair of basis vectors $v_{\tau} \in \Lambda^{-1} \operatorname{Lie}(n-1), v_{\tau^{\prime}} \in \Lambda^{-1} \operatorname{Lie}(m-1)$, the decomposition of the pre-Lie product

$$
\left(T_{1} \otimes b_{j_{1}} v_{\tau}\left(b_{1}, \ldots, \widehat{b_{j_{1}}}, \ldots, b_{n}\right)\right) \bullet\left(T_{2} \otimes b_{j_{2}} v_{\tau^{\prime}}\left(b_{1}, \ldots, \widehat{b_{j_{2}}}, \ldots, b_{m}\right)\right)
$$

in the basis 10.13 does not involve vectors of the form

$$
V_{i}^{\circ-\circ}(T) \otimes b_{i} b_{i+1} v_{\tau}\left(b_{1}, \ldots, b_{i-1}, b_{i+2}, \ldots, b_{n}\right) .
$$

These observations imply that, if the decomposition of the element $\operatorname{Av}^{-1}(\alpha)$ in the basis 10.13 involves a vector of the form $(10.16)$, then

$$
\operatorname{Av}^{-1}(\alpha) \bullet^{\prime} \mathrm{Av}^{-1}(\alpha) \neq 0 .
$$

On the other hand, $\mathrm{Av}^{-1}(\alpha)$ is a Maurer-Cartan element of 10.12 . Thus, the proposition follows.

10.2. Proof of Corollary 1.2. In this section, we give a proof of Corollary 1.2 of Theorem 1.1 stated in the Introduction.

Let us assume that the quasi-isomorphism

$$
F: \mathrm{Ger}_{\infty} \rightarrow \mathrm{Br}
$$

is compatible with the $\mathrm{Tw}$-coalgebra structures on $\mathrm{Ger}_{\infty}$ and $\mathrm{Br}$. This means that the diagram

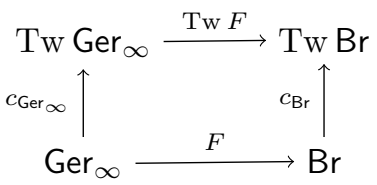

commutes.

Following Theorem 3.8 and using the Maurer-Cartan element $\alpha$ of $C^{\bullet}(A)$, we form an operad map

$$
\mathfrak{a}_{A}^{\alpha}: \operatorname{Tw} \mathrm{Br} \rightarrow \operatorname{End}_{C} \cdot(A)^{\alpha} .
$$

Unfolding definitions, we see that the composition

$$
\mathfrak{a}_{A}^{\alpha} \circ \operatorname{Tw}(F) \circ c_{\operatorname{Ger}_{\infty}}: \operatorname{Ger}_{\infty} \rightarrow \operatorname{End}_{C} \cdot(A)^{\alpha}
$$

gives us the $\mathrm{Ger}_{\infty}$-structure $F_{A}^{\alpha}$ and the composition

$$
\mathfrak{a}_{A}^{\alpha} \circ c_{\mathrm{Br}} \circ F: \operatorname{Ger}_{\infty} \rightarrow \operatorname{End}_{C} \cdot(A)^{\alpha}
$$


give us the $\mathrm{Ger}_{\infty}$-structure $F_{A^{\alpha}}$.

Thus, since diagram 10.19 commutes, the $\mathrm{Ger}_{\infty}$-structures $F_{A^{\alpha}}$ and $F_{A}^{\alpha}$ coincide and the second statement of Corollary 1.2 is proved.

Let us now consider the case when

$$
F: \mathrm{Ger}_{\infty} \rightarrow \mathrm{Br}
$$

is an arbitrary solution of Deligne's conjecture (not necessarily compatible with Tw-coalgebra structures).

Due to Theorem 1.1. $F$ is homotopy equivalent to a quasi-isomorphism

$$
F^{\prime}: \mathrm{Ger}_{\infty} \rightarrow \mathrm{Br}
$$

that is compatible with the $\mathrm{Tw}$-coalgebra structures on $\mathrm{Ger}_{\infty}$ and $\mathrm{Br}$.

Hence diagram 10.19) commutes up to homotopy. Therefore, the two (possibly different) Ger $\infty_{\infty}$-structures $F_{A^{\alpha}}$ and $F_{A}^{\alpha}$ are indeed homotopy equivalent.

Corollary 1.2 is proved.

\section{Appendix A. The Operad Ger}

A Gerstenhaber algebra is a graded vector space $V$ equipped with a commutative (and associative) product (without identity) and a degree -1 binary operation $\{$,$\} which satisfies the following relations:$

$$
\begin{gathered}
\left\{v_{1}, v_{2}\right\}=(-1)^{\left|v_{1}\right|\left|v_{2}\right|}\left\{v_{2}, v_{1}\right\}, \\
\left\{v, v_{1} v_{2}\right\}=\left\{v, v_{1}\right\} v_{2}+(-1)^{\left|v_{1}\right||v|+\left|v_{1}\right|} v_{1}\left\{v, v_{2}\right\}, \\
\left\{\left\{v_{1}, v_{2}\right\}, v_{3}\right\}+(-1)^{\left|v_{1}\right|\left(\left|v_{2}\right|+\left|v_{3}\right|\right)}\left\{\left\{v_{2}, v_{3}\right\}, v_{1}\right\}+(-1)^{\left|v_{3}\right|\left(\left|v_{1}\right|+\left|v_{2}\right|\right)}\left\{\left\{v_{3}, v_{1}\right\}, v_{2}\right\}=0 .
\end{gathered}
$$

To define spaces of the operad Ger governing Gerstenhaber algebras we introduce the free Gerstenhaber algebra $\operatorname{Ger}_{n}$ in $n$ dummy variables $a_{1}, a_{2}, \ldots, a_{n}$ of degree 0 . Next we set $\operatorname{Ger}(0)=\mathbf{0}$ and $\operatorname{Ger}(1)=\mathbb{K}$. And then we declare that, for $n \geq 2, \operatorname{Ger}(n)$ is spanned by monomials of $\operatorname{Ger}_{n}$ in which each dummy variable $a_{i}$ appears exactly once.

The symmetric group $S_{n}$ acts on $\operatorname{Ger}(n)$ in the obvious way by permuting the dummy variables. It is also clear how to define elementary insertions.

Example A.1. Let us consider the monomials $u=\left\{a_{2}, a_{3}\right\} a_{1}\left\{a_{4}, a_{5}\right\} \in \operatorname{Ger}(5)$ and $w=\left\{a_{1}, a_{2}\right\} \in \operatorname{Ger}(2)$ and compute the insertions $u \circ_{2} w, u \circ_{4} w$ and $w \circ_{1} u$. We get

$$
\begin{aligned}
u \circ_{2} w= & -\left\{\left\{a_{2}, a_{3}\right\}, a_{4}\right\} a_{1}\left\{a_{5}, a_{6}\right\} \\
u \circ_{4} w= & \left\{a_{2}, a_{3}\right\} a_{1}\left\{\left\{a_{4}, a_{5}\right\}, a_{6}\right\} \\
w \circ_{1} u= & \left\{\left\{a_{2}, a_{3}\right\} a_{1}\left\{a_{4}, a_{5}\right\}, a_{6}\right\}=\left\{a_{6},\left\{a_{2}, a_{3}\right\} a_{1}\left\{a_{4}, a_{5}\right\}\right\} \\
= & \left\{a_{6},\left\{a_{2}, a_{3}\right\}\right\} a_{1}\left\{a_{4}, a_{5}\right\}-\left\{a_{2}, a_{3}\right\}\left\{a_{6}, a_{1}\right\}\left\{a_{4}, a_{5}\right\} \\
& -\left\{a_{2}, a_{3}\right\} a_{1}\left\{a_{6},\left\{a_{4}, a_{5}\right\}\right\} .
\end{aligned}
$$

It is easy to see that the operad Ger is generated by the vectors $a_{1} a_{2},\left\{a_{1}, a_{2}\right\} \in \operatorname{Ger}(2)$. 
FIG. B.1. A brace tree $T \in \mathcal{T}(4)$

\section{Appendix B. Action of the operad Tw BT on the Hochschild COCHAin COMPlex of AN}

$$
A_{\infty} \text {-ALGEBRA }
$$

Let $A$ be a cochain complex. We form another cochain complex

$$
C^{\bullet}(A)=\bigoplus_{m \geq 0} \mathbf{s}^{m} \operatorname{Hom}\left(A^{\otimes m}, A\right)
$$

with the differential $\partial_{A}$ coming from $A$.

Let us show that (B.1) is a naturally an algebra over BT. To define an action BT on B.1 we observe that the collection

$$
\left\{\operatorname{Hom}\left(A^{\otimes m}, A\right)\right\}_{m \geq 0}
$$

is an naturally an operad, i.e. the endomorphism operad of $A$.

Using this observation, we will construct an auxiliary map

$$
\varrho: \bigoplus_{N \geq 1}\left(\mathrm{BT}(N) \otimes\left(C^{\bullet}(A)\right)^{\otimes N}\right)_{S_{N}} \rightarrow A
$$

Given a brace tree $T \in \mathcal{T}(N)$ and $N$ homogeneous vectors

$$
P_{i} \in \mathbf{s}^{m_{i}} \operatorname{Hom}\left(A^{\otimes m_{i}}, A\right), \quad 1 \leq i \leq N
$$

we decorated non-root vertices of $T$ with vectors (B.4) following this rule: the vertex with label $i$ is decorated by the vector $P_{i}$.

We say that such a decoration is admissible if for every $1 \leq i \leq N$ the vertex with label $i$ has exactly $m_{i}$ incoming edges. Otherwise, we say that the decoration is inadmissible. In particular, if a decoration is admissible, then each leaf of $T$ is decorated by a vector in

$$
\operatorname{Hom}\left(A^{\otimes 0}, A\right)=A .
$$

Since 3.2 is an operad, each brace tree $T \in \mathcal{T}(N)$ with an admissible decoration gives us a vector in $A$. We denote this vector in $A$ by

$$
\mathfrak{m}\left(T ; P_{1}, P_{2}, \ldots, P_{N}\right) .
$$

For example, if $T$ the brace tree depicted on figure B.1. $P_{1} \in \operatorname{sHom}(A, A), P_{2} \in A, P_{3} \in \mathbf{s}^{2} \operatorname{Hom}\left(A^{\otimes 2}, A\right)$, and $P_{4} \in A$ then we have

$$
\mathfrak{m}\left(T ; P_{1}, P_{2}, P_{3}, P_{4}\right)=P_{3}\left(P_{1}\left(P_{2}\right), P_{4}\right) .
$$

We can now define the map $\varrho$ B.3.

If $T$ is a brace tree in $\mathcal{T}(N)$ and cochains $P_{1}, P_{2}, \ldots, P_{N}$ give us an inadmissible decoration of $T$ then we set

$$
\varrho\left(T, P_{1}, \ldots, P_{N}\right)=0 .
$$




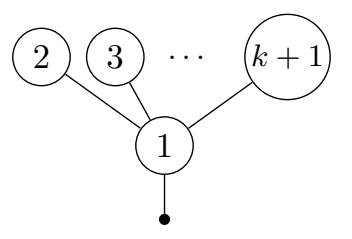

FIG. B.2. The brace corolla $T_{k} \in \mathcal{T}(k+1)$

Otherwise, we declare that

$$
\varrho\left(T, P_{1}, \ldots, P_{N}\right):=(-1)^{\varepsilon\left(T ; P_{1}, \ldots, P_{N}\right)} \mathfrak{m}\left(T ; P_{1}, \ldots, P_{N}\right)
$$

where the sign factor $(-1)^{\varepsilon\left(T ; P_{1}, \ldots, P_{N}\right)}$ comes from permutation on the set

$$
\{E(T) \backslash\{\text { root edge }\}\} \sqcup\left\{P_{1}, \ldots, P_{N}\right\}
$$

which we perform when we decorate the tree $T$ with vectors $P_{1}, P_{2}, \ldots, P_{N}$.

For example, if $T$ is the brace tree depicted on figure B.1. $P_{1} \in \operatorname{sHom}(A, A), P_{2} \in A, P_{3} \in \mathbf{s}^{2} \operatorname{Hom}\left(A^{\otimes 2}, A\right)$, and $P_{4} \in A$ then

$$
\varepsilon\left(T ; P_{1}, \ldots, P_{N}\right)=\left|P_{3}\right|\left(\left|P_{1}\right|+\left|P_{2}\right|\right)+3\left|P_{3}\right|+2\left|P_{1}\right|+\left|P_{2}\right| .
$$

The sign factor $(-1)^{\left|P_{3}\right|\left(\left|P_{1}\right|+\left|P_{2}\right|\right)}$ appears because we need to switch from the order $\left(P_{1}, P_{2}, P_{3}, P_{4}\right)$ to the order $\left(P_{3}, P_{1}, P_{2}, P_{4}\right)$; the sign factor $(-1)^{3\left|P_{3}\right|}$ appears because $P_{3}$ "jumps over" three edges of brace tree $T$. Similarly, $P_{1}$ (resp. $P_{2}$ ) "jumps over" two edges (resp. one edge) of the brace tree $T$.

We can now define how a brace tree $T \in \mathcal{T}(n)$ acts on $n$ homogeneous vectors

$$
P_{i} \in \mathbf{s}^{m_{i}} \operatorname{Hom}\left(A^{\otimes m_{i}}, A\right), \quad 1 \leq i \leq n .
$$

For this purpose, we form the linear combinations $(k \geq 0)$

$$
T_{k} \circ_{1} T
$$

where $T_{k}$ is the brace corolla shown on figure B.2, and

$$
k=\sum_{i=1}^{n} m_{i}+1-n
$$

Next we set

$$
T\left(P_{1}, \ldots, P_{n} ; a_{1}, \ldots, a_{k}\right)=\varrho\left(T_{k} \circ_{1} T, P_{1}, \ldots, P_{n}, a_{1}, \ldots, a_{k}\right),
$$

where $a_{1}, \ldots, a_{k}$ are viewed as vectors in

$$
\operatorname{Hom}\left(A^{\otimes 0}, A\right) .
$$

Note that, the vectors $a_{1}, \ldots, a_{k}$ will decorate leaves (of brace trees in the linear combination $T_{k} \circ_{1} T$ ) with labels $n+1, n+2, \ldots, n+k$. Moreover, if equation B.10 were not satisfied then all decorations of $T_{k} \circ_{1} T$ would be inadmissible.

Tedious but straightforward computations show that formula $(\mathrm{B} .11)$ indeed defines an action of the operad BT on B.1. 


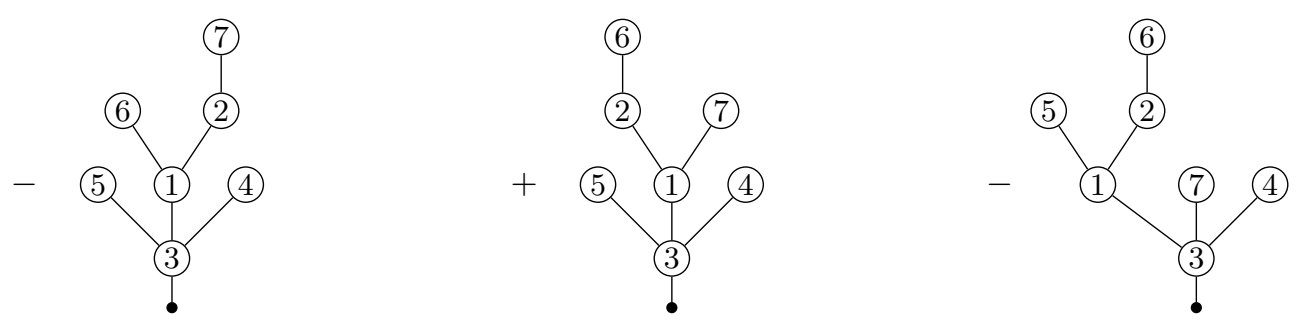

FIG. B.3. The brace trees which contribute to $T\left(P_{1}, P_{2}, P_{3}, P_{4}\right)$

Example B.1. Let $T$ be the brace tree in $\mathcal{T}(4)$ depicted on figure B.1 and

$$
\begin{aligned}
& P_{1} \in \mathbf{s}^{2} \operatorname{Hom}(A \otimes A, A), \quad P_{2} \in \mathbf{s} \operatorname{Hom}(A, A), \\
& P_{3} \in \mathbf{s}^{3} \operatorname{Hom}\left(A^{\otimes 3}, A\right), \quad P_{4} \in \operatorname{Hom}\left(A^{\otimes 0}, A\right)=A .
\end{aligned}
$$

The vector $T\left(P_{1}, P_{2}, P_{3}, P_{4}\right)$ belongs to

$$
\mathbf{s}^{3} \operatorname{Hom}\left(A^{\otimes 3}, A\right)
$$

The linear combination $T_{3} \circ_{1} T$ contains a lot of terms. On figure B.3 we list all brace trees (with signs) which get admissible decorations by the vectors

$$
P_{1}, P_{2}, P_{3}, P_{4}, a_{1}, a_{2}, a_{3}
$$

Thus we get

$$
\begin{aligned}
T\left(P_{1}, P_{2}, P_{3}, P_{4}\right)\left(a_{1}, a_{2}, a_{3}\right)= & -(-1)^{\varepsilon_{1}} P_{3}\left(a_{1}, P_{1}\left(a_{2}, P_{2}\left(a_{3}\right)\right), P_{4}\right)+ \\
& (-1)^{\varepsilon_{2}} P_{3}\left(a_{1}, P_{1}\left(P_{2}\left(a_{2}\right), a_{3}\right), P_{4}\right)-(-1)^{\varepsilon_{3}} P_{3}\left(P_{1}\left(a_{1}, P_{2}\left(a_{2}\right)\right), a_{3}, P_{4}\right),
\end{aligned}
$$

where

$$
\begin{aligned}
& \varepsilon_{1}=\varepsilon\left(P_{3}, a_{1}, P_{1}, a_{2}, P_{2}, a_{3}, P_{4}\right)+6\left|P_{3}\right|+5\left|a_{1}\right|+4\left|P_{1}\right|+3\left|a_{2}\right|+2\left|P_{2}\right|+\left|a_{3}\right|, \\
& \varepsilon_{2}=\varepsilon\left(P_{3}, a_{1}, P_{1}, P_{2}, a_{2}, a_{3}, P_{4}\right)+6\left|P_{3}\right|+5\left|a_{1}\right|+4\left|P_{1}\right|+3\left|P_{2}\right|+2\left|a_{2}\right|+\left|a_{3}\right|, \\
& \varepsilon_{3}=\varepsilon\left(P_{3}, P_{1}, a_{1}, P_{2}, a_{2}, a_{3}, P_{4}\right)+6\left|P_{3}\right|+5\left|P_{1}\right|+4\left|a_{1}\right|+3\left|P_{2}\right|+2\left|a_{2}\right|+\left|a_{3}\right|,
\end{aligned}
$$

and $\varepsilon(\ldots)$ is the sign of the permutation of vectors $P_{1}, P_{2}, P_{3}, P_{4}, a_{1}, a_{2}, a_{3}$ from their standard position B.13).

Since BT receives the operad map 8.1 from $\Lambda$ Lie, the cochain complex (B.1) is naturally a $\Lambda$ Lie algebra. The bracket is the famous Gerstenhaber bracket introduced in [13].

Let us observe that the cochain complex $C^{\bullet}(A)$ B.1 is equipped with the obvious decreasing filtration:

$$
\mathcal{F}_{q} C^{\bullet}(A)=\left\{P \in C^{\bullet}(A) \mid P\left(a_{1}, a_{2}, \ldots, a_{k}\right)=0 \forall k \leq q\right\}
$$

and the action of $\mathrm{BT}$ on $C^{\bullet}(A)$ is compatible with this filtration. Thus we may apply the general procedure of twisting described in Section 3 to the completion

$$
\hat{C}^{\bullet}(A):=\prod_{m \geq 0} \mathbf{s}^{m} \operatorname{Hom}\left(A^{\otimes m}, A\right)
$$

of the BT-algebra $C^{\bullet}(A)$ with respect to the filtration B.15. 
According to Theorem 3.10, filtered Tw BT-algebra structures on $\hat{C}^{\bullet}(A)$ are in bijection with MaurerCartan elements $\alpha$ in $\hat{C}^{\bullet}(A)$ of the form

$$
\alpha=\sum_{k \geq 2} \alpha_{k}, \quad \alpha_{k} \in \mathbf{s}^{k} \operatorname{Hom}\left(A^{\otimes k}, A\right)
$$

i.e. flat $A_{\infty}$-structures on the cochain complex $A$.

Moreover, for every Maurer-Cartan element (B.17), the cochain complex $\hat{C}^{\bullet}(A)$ with the twisted differential 3.22 is exactly the (completed) Hochschild cochain complex of the $A_{\infty}$-algebra $A$.

Thus we conclude that, for every flat $A_{\infty}$-algebra $A$, its completed Hochschild cochain complex is equipped with a natural action of the operad Tw BT and hence with a natural action of the operad Br.

Remark B.2. Sometimes the Maurer-Cartan element B.17) satisfies the condition

$$
\alpha_{k}=0
$$

for all $k \geq N$ for some given number $N$. (For example, if $A$ is an associative algebra.) In this case, the action of $\mathrm{Tw} \mathrm{BT}$ (and hence the action of $\mathrm{Br}$ ) is well defined before completing the Hochschild cochain complex.

Appendix C. The equivalent definition of the pre-Lie product • on Conv $(Q, P)$ in terms of COINVARIANTS

Let us assume that the pseudo-cooperad $Q$ satisfies the following property.

Property C.1. For each $n$ the graded vector space $Q(n)$ is finite dimensional.

Due to this property we have

$$
\operatorname{Conv}(Q, P) \cong \prod_{n \geq 0}\left(P(n) \otimes Q^{*}(n)\right)^{S_{n}} .
$$

where $Q^{*}(n)$ denotes the linear dual of the vector space $Q(n)$.

The collection $Q^{*}:=\left\{Q^{*}(n)\right\}_{n \geq 0}$ is naturally a pseudo-operad and we can express the pre-Lie structure 2.20 in terms of elementary insertions on $P$ and $Q^{*}$. Namely, given two vectors

$$
X=\sum_{n \geq 0} v_{n} \otimes w_{n}, \quad X^{\prime}=\sum_{n \geq 0} v_{n}^{\prime} \otimes w_{n}^{\prime}
$$

in

$$
\prod_{n \geq 0}\left(P(n) \otimes Q^{*}(n)\right)^{S_{n}}
$$

we have

$$
X \bullet X^{\prime}=\sum_{n \geq 1, m \geq 0}(-1)^{\left|v_{m}^{\prime}\right|\left|w_{n}\right|} \sum_{\sigma \in \operatorname{Sh}_{m, n-1}} \sigma\left(v_{n} \circ_{1} v_{m}^{\prime}\right) \otimes \sigma\left(w_{n} \circ_{1} w_{m}^{\prime}\right) .
$$

Let us now observe that the formula

$$
\operatorname{Av}(v \otimes w)=\sum_{\sigma \in S_{n}} \sigma(v) \otimes \sigma(w)
$$

defines a $\mathbb{K}$-linear map

$$
\mathrm{Av}: P(n) \otimes Q^{*}(n) \rightarrow\left(P(n) \otimes Q^{*}(n)\right)^{S_{n}}
$$


Furthermore, since $\mathbb{K}$ has characteristic zero, Av induces an isomorphism (which we denote by the same letter):

$$
\mathrm{Av}:\left(P(n) \otimes Q^{*}(n)\right)_{S_{n}} \stackrel{\cong}{\longrightarrow}\left(P(n) \otimes Q^{*}(n)\right)^{S_{n}}
$$

from the space

$$
\left(P(n) \otimes Q^{*}(n)\right)_{S_{n}}
$$

of $S_{n}$-coinvariants to the space $\left(P(n) \otimes Q^{*}(n)\right)^{S_{n}}$ of invariants.

We have the following proposition.

Proposition C.2. Let $v, v^{\prime}, w, w^{\prime}$ be homogeneous vectors in $P(n), P(m), Q(n)$, and $Q^{\prime}(m)$, respectively. Then the formula

$$
(v \otimes w) \bullet^{\prime}\left(v^{\prime} \otimes w^{\prime}\right)=\sum_{i=1}^{n}(-1)^{\left|v^{\prime}\right||w|} v \circ_{i} v^{\prime} \otimes w \circ_{i} w^{\prime}
$$

defines a binary operation on

$$
\bigoplus_{n \geq 0}\left(P(n) \otimes Q^{*}(n)\right)_{S_{n}}
$$

This operation extends in the obvious way to the infinite sum

$$
\prod_{n \geq 0}\left(P(n) \otimes Q^{*}(n)\right)_{S_{n}}
$$

and

$$
\operatorname{Av}\left((v \otimes w) \bullet\left(v^{\prime} \otimes w^{\prime}\right)\right)=\operatorname{Av}(v \otimes w) \bullet \operatorname{Av}\left(v^{\prime} \otimes w^{\prime}\right) .
$$

Proof. Since the map C.4 is an isomorphism of graded vector spaces, it suffices to prove that equation C.8 holds.

This equation can be verified by a straightforward computation using the obvious identity ${ }^{15}$

$$
\sum_{\sigma \in S_{n}} \sigma(v)=\sum_{\sigma^{\prime} \in S_{\{2,3, \ldots, n\}}} \sum_{i=1}^{n} \sigma^{\prime} \circ(1,2, \ldots, i)(v)
$$

and properties of the elementary insertions.

Proposition C.2 implies that the map (C.4) establishes an isomorphism between the pre-Lie algebra C.1) with the pre-Lie product (C.2) and the pre-Lie algebra (C.7) with the pre-Lie product given by equation C.6.

\section{Appendix D. A Lemma on a filtered Complex}

Lemma D.1. Let $C$ be a cochain complex with a complete descending filtration which is bounded above. I.e.,

$$
C=\mathcal{F}_{-N} \supset \mathcal{F}_{-N+1} \supset \cdots \supset \mathcal{F}_{0} \supset \mathcal{F}_{1} \supset \cdots
$$

and $C=\lim _{p} C / \mathcal{F}_{p}$. If the associated graded complex $\operatorname{Gr} C$ is acyclic then so is $C$.

\footnotetext{
${ }^{15}$ Here $S_{\{2,3, \ldots, n\}}$ is the subgroup of elements $\sigma \in S_{n}$ satisfying the condition $\sigma(1)=1$.
} 


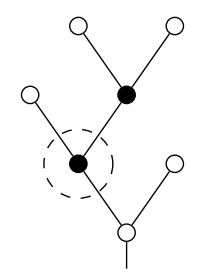

FIG. E.1. The first neutral vertex of a brace tree.

Proof. Suppose $c \in C$ is a cocycle. Then there exists $j$ such that $c \in \mathcal{F}_{j}$. Then by acyclicity of the associated graded complex, there is a $b_{j} \in \mathcal{F}_{j}$ such that $c-d b_{j} \in \mathcal{F}_{j+1}$. Proceed in this manner to define $b_{j+1}, b_{j+2}$ etc. Then set $b:=\sum_{j} b_{j}$. The sum converges since $C$ is complete with respect to the filtration. Again by completeness

$$
c-d b=\lim _{N \rightarrow \infty} c-\sum_{j \leq N} d b_{j}=0 .
$$

Hence $c$ is exact.

\section{Appendix E. Br is a Homotopy Tw FiXed Point - COMBinatorial ARGUMEnt}

We showed in Section 9.3 above that the operad $\mathrm{Br}$ is a homotopy fixed point of $\mathrm{Tw}$, i. e., that $\mathrm{Tw} \mathrm{Br} \rightarrow \mathrm{Br}$ is a quasi-isomorphism. One may give an alternative proof of that fact by an elementary combinatorial argument, which we briefly sketch in this appendix.

Elements of $\mathrm{Tw} \mathrm{Br}$ can be seen as series of brace trees, for which some of the labeled vertices have been colored in, say, gray. The differential is schematically depicted in Figure E.4. The number of neutral ("black") vertices of brace trees yields a descending complete filtration

$$
\mathrm{Tw} \mathrm{Br}=\mathcal{F}^{0} \supset \mathcal{F}^{1} \supset \mathcal{F}^{2} \supset \cdots
$$

where $\mathcal{F}^{p}$ is composed of series in trees with $\geq p$ neutral (black) vertices. Let us consider the associated graded Gr $\mathcal{F}$. Its differential misses those terms of Figure E.4 that produce a black vertex. We claim that $V_{p}:=\mathcal{F}^{p} / \mathcal{F}^{p+1}$ is acyclic for $p \geq 1$. To show this we need to use additional notation. For a brace tree, the first neutral vertex is the one hit first when going around he tree in clockwise order, see Figure E.1. We filter $V_{p}$ by the number of valence zero gray vertices attached to the very left of the first internal vertex, see Figure E.2. Taking a spectral sequence, the first differential increases that number by one, see Figure E.3. It is easy to see that the cohomology under this differential is zero. It follows that $V_{p}$ is acyclic as claimed. Hence the projection $\operatorname{Gr} \mathcal{F} \rightarrow \mathcal{F}^{0} / \mathcal{F}^{1}$ is a quasi-isomorphism. From this one can see that also the projection $\mathrm{Tw} \mathrm{Br} \rightarrow \mathrm{Tw} \mathrm{BT}$ is a quasi-isomorphism. But since $\mathrm{Br} \rightarrow \mathrm{Tw} \mathrm{BT}$ is a quasi-isomorphism by section 9.2 , we are done.

\section{REFERENCES}

[1] M. Batanin and M. Markl, Crossed interval groups and operations on the Hochschild cohomology, arXiv:0803.2249.

[2] C. Berger and B. Fresse, Combinatorial operad actions on cochains, Math. Proc. Cambridge Philos. Soc., 137, 1 (2004) 135-174.

[3] C. Berger and I. Moerdijk, Axiomatic homotopy theory for operads, Comment. Math. Helv. 78, 4 (2003) 805-831; arXiv:math/0206094

[4] J. Chuang and A. Lazarev, L-infinity maps and twistings, Homology Homotopy Appl. 13, 2 (2011) 175-195. 


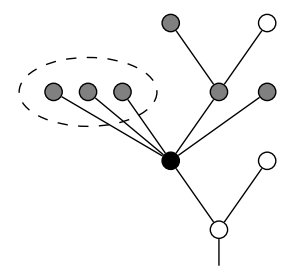

FIG. E.2. The filtration on $V_{p}$ we use comes from the number of valence zero gray vertices attached to to the very left of the first neutral vertex. In this example, that number is three.

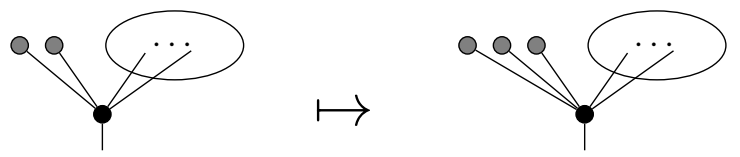

FIG. E.3. The differential increases the number of valence zero gray vertices attached to to the very left of the first neutral vertex by at most one. The component that increases this number by exactly one is shown here. Note that if this number is odd, the differential acts as zero.

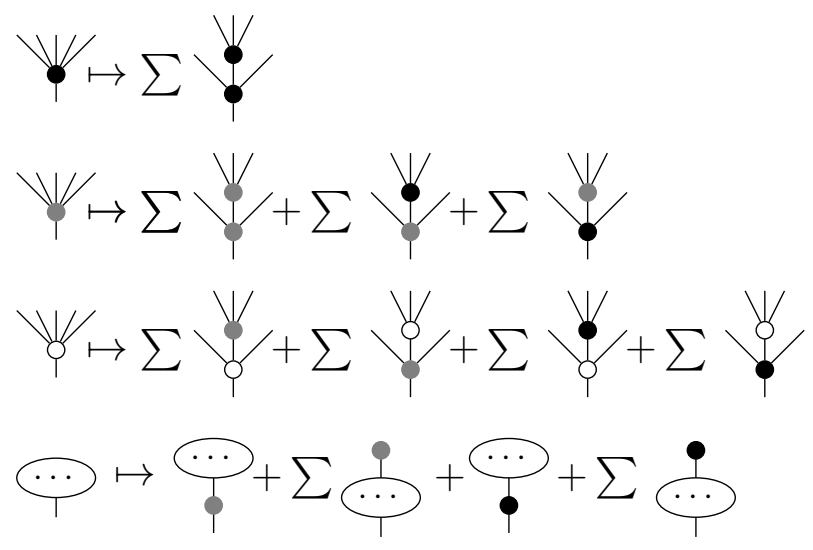

FIG. E.4. A pictorial description of the differential on Tw Br. On the associated graded Gr $\mathcal{F}$ the terms that create black vertices (i. e., the first row and the last two terms in the remaining rows) are absent. The terms depicted in Figure E.3 are contained in the second term of the last row.

[5] J. Chuang and A. Lazarev, Combinatorics and formal geometry of the master equation, Lett. Math. Phys. 103, 1 (2013) 79-112; arXiv:1205.5970.

[6] V.A. Dolgushev, Covariant and equivariant formality theorems, Adv. Math. 191, 1 (2005)147-177.

[7] V.A. Dolgushev, A proof of Tsygan's formality conjecture for an arbitrary smooth manifold, Ph.D. thesis, M.I.T. (2005); arXiv:math/0504420

[8] V.A. Dolgushev and C.L. Rogers, Notes on Algebraic Operads, Graph Complexes, and Willwacher's Construction, Mathematical aspects of quantization, 25-145, Contemp. Math., 583, Amer. Math. Soc., Providence, RI, 2012; arXiv:1202.2937

[9] V.A. Dolgushev, D.E. Tamarkin, and B.L. Tsygan, Proof of Swiss Cheese Version of Deligne's Conjecture, Int. Math. Res. Notices 2011 (2011) 4666-4746.

[10] B. Fresse, Lie theory of formal groups over an operad, J. of Algebra 202 (1998), 455-511.

[11] B. Fresse, Koszul duality of operads and homology of partition posets, in "Homotopy theory and its applications (Evanston, 2002)", Contemp. Math. 346 (2004) 115-215. 
[12] I. Galvez-Carrillo, A. Tonks, and B. Vallette, Homotopy Batalin-Vilkovisky algebras, J. Noncomm. Geom. 6, 3 (2012) 539-602; arXiv:0907.2246

[13] M. Gerstenhaber, The cohomology structure of an associative ring, Annals of Math., 78 (1963) 267-288.

[14] E. Getzler and J.D.S. Jones, Operads, homotopy algebra and iterated integrals for double loop spaces, hep-th/9403055

[15] V. Ginzburg and M. Kapranov, Koszul duality for operads, Duke Math. J. 76, 1 (1994) 203-272.

[16] V. Hinich, Homological algebra of homotopy algebras, Comm. Algebra 25 (1997), 3291-3323.

[17] V. Hinich, Erratum to "Homological algebra of homotopy algebras", arXiv:math/0309453

[18] V. Hinich, Tamarkin's proof of Kontsevich formality theorem, Forum Math. 15, 4 (2003) 591-614; math.QA/0003052

[19] M. Kapranov and Y. Manin, Modules and Morita theorem for operads, Amer. J. Math. 123 (2001), 811-838.

[20] M. Kontsevich, Operads and motives in deformation quantization, Lett. Math. Phys. 48, 1 (1999) 35-72.

[21] M. Kontsevich and Y. Soibelman, Deformations of algebras over operads and the Deligne conjecture, Proceedings of the Moshé Flato Conference Math. Phys. Stud. 21, 255-307, Kluwer Acad. Publ., Dordrecht, 2000.

[22] J.-L. Loday and B. Vallette, Algebraic Operads, Grundlehren der mathematischen Wissenschaften, 346, Springer-Verlag 2012.

[23] M. Markl, Operads and PROPs, Handbook of algebra. Vol. 5, 87-140, Elsevier/North-Holland, Amsterdam, 2008; arXiv:math/0601129

[24] M. Markl, Distributive laws and Koszulness, Ann. Inst. Fourier (Grenoble) 46, 2 (1996) 307-323.

[25] M. Markl, S. Shnider, and J. Stasheff, Operads in algebra, topology and physics, Mathematical Surveys and Monographs, 96. AMS, Providence, RI, 2002. $\mathrm{x}+349$ pp.

[26] J. E. McClure and J. H. Smith, A solution of Deligne's Hochschild cohomology conjecture, Contemp. Math. 293 (2002) 153-193, Amer. Math. Soc., Providence, RI; math.QA/9910126.

[27] J. E. McClure and J. H. Smith, Multivariable cochain operations and little $n$-cubes, J. Amer. Math. Soc. 16, 3 (2003) 681-704.

[28] S. Merkulov and B. Vallette, Deformation theory of representations of prop(erad)s, I, II, J. Reine Angew. Math. 634 (2009), 51-106. and J. Reine Angew. Math. 636 (2009), 123-174, arXiv:0707.0889

[29] D. Quillen, Rational homotopy theory, Ann. of Math. (2) 90 (1969) 205-295.

[30] D. Tamarkin, Formality of chain operad of little discs, Lett. Math. Phys. 66, 1-2 (2003) 65-72.

[31] D. Tamarkin, What do DG categories form? Compos. Math. 143, 5 (2007) 1335-1358; math.CT/0606553

[32] A.A. Voronov, Homotopy Gerstenhaber algebras, Proceedings of the Moshé Flato Conference Math. Phys. Stud., 22, 307-331. Kluwer Acad. Publ., Dordrecht, 2000.

[33] T. Willwacher, M. Kontsevich's graph complex and the Grothendieck-Teichmüller Lie algebra, arXiv:1009.1654

[34] A. Yekutieli, Continuous and Twisted L-infinity Morphisms, J. Pure Appl. Algebra 207, (2006), 575-606.

Department of Mathematics, Temple University,

WACHMAN HaLl RM. 638

1805 N. Broad St.,

Philadelphia, PA, 19122 USA

E-mail address: vald@temple.edu

UNIVERSITY OF ZÜRICH,

Institute of MATHEMATICS, WINTERTHURERSTRASSE 190, 8057 ZÜrich, SwitzerLAND

E-mail address: thomas.willwacher@math.uzh.ch 